\title{
The supermassive black hole in Centaurus A: a benchmark for gas kinematical measurements ${ }^{\star}$
}

\author{
A. Marconi ${ }^{1}$, G. Pastorini ${ }^{2}$, F. Pacini ${ }^{2}$, D. J. Axon ${ }^{3,4}$, A. Capetti ${ }^{5}$, D. Macchetto ${ }^{6,7}$, \\ A. M. Koekemoer ${ }^{6}$, and E. J. Schreier ${ }^{8}$
}

1 INAF - Osservatorio Astrofisico di Arcetri Largo E. Fermi 5, 50125 Firenze, Italy e-mail: marconi@arcetri.astro.it

2 Dipartimento di Astronomia e Scienza dello Spazio, Università degli Studi di Firenze Largo E. Fermi 2, 50125 Firenze, Italy e-mail: [guia;pacini]@arcetri.astro.it

3 Department of Physics, Rochester Institute of Technology, 85 Lomb Memorial Drive, Rochester, NY 14623, USA e-mail: djasps@rit.edu

4 Department of Physical Sciences, University of Hertfordshire, Hatfield AL10 9AB, UK

5 INAF - Osservatorio Astronomico di Torino, Strada Osservatorio 20, 10025 Pino Torinese, Italy e-mail: capetti@to.astro.it

${ }^{6}$ Space Telescope Science Institute 3700 San Martin Drive, Baltimore, MD 21218, USA e-mail: macchetto@stsci.edu, koekemoe@stsci.edu

7 Affiliated with ESA's Space Telescope Division

8 Associated Universities, Inc. Suite 7301400 16th Street, NW Washington, DC 20036, USA e-mail: ejs@aui.edu

Received 19 July 2005 / Accepted 12 November 2005

ABSTRACT

We present new HST Space Telescope Imaging Spectrograph observations of the nearby radio galaxy NGC 5128 (Centaurus A). The bright emission line with longest wavelength accessible from HST, [S III] $\lambda 9533 \AA$, was used to study the kinematics of the ionized gas in the nuclear region with a 0 '. 1 spatial resolution. The STIS data were analized in conjunction with the ground-based near-infrared Very Large Telescope ISAAC spectra used by Marconi et al. (2001, ApJ, 549, 915) to infer the presence of a supermassive black hole and measure its mass. The two sets of data have spatial resolutions differing by almost a factor of five but provide independent and consistent measures of the $\mathrm{BH}$ mass, which are in agreement with our previous estimate based on the ISAAC data alone. The gas kinematical analysis provides a mass of $M_{\mathrm{BH}}=(1.1 \pm 0.1) \times 10^{8} M_{\odot}$ for an assumed disk inclination of $i=25 \mathrm{deg}$ or $M_{\mathrm{BH}}=(6.5 \pm 0.7) \times 10^{7} M_{\odot}$ for $i=35 \mathrm{deg}$, the largest $i$ value allowed by the data. We performed a detailed analysis of the effects on $M_{\mathrm{BH}}$ of the intrinsic surface brightness distribution of the emission line, a crucial ingredient in the gas kinematical analysis. We estimate that the associated systematic errors are no larger than 0.08 in log $M_{\mathrm{BH}}$, comparable with statistical errors and indicating that the method is robust. However, the intrinsic surface brightness distribution has a large impact on the value of the gas velocity dispersion. A mismatch between the observed and model velocity dispersion is not necessarily an indication of non-circular motions or kinematically hot gas, but is as easily due to an inaccurate computation arising from too course a model grid, or the adoption of an intrinsic brightness distribution which is too smooth. The observed velocity dispersion in our spectra can be matched with a circularly rotating disk and also the observed line profiles and the higher order moments in the Hermite expansion of the line profiles, $h_{3}$ and $h_{4}$, are consistent with emission from such a disk. To our knowledge, Centaurus A is the first external galaxy for which reliable BH mass measurements from gas and stellar dynamics are available and, as in the case of the Galactic Center, the $M_{\mathrm{BH}}$ gas kinematical estimate is in good agreement with that from stellar dynamics. The BH mass in Centaurus A is in excellent agreement with the correlation with infrared luminosity and mass of the host spheroid but is a factor $\sim 2-4$ above the one with the stellar velocity dispersion. But this disagreement is not large if one takes into account the intrinsic scatter of the $M_{\mathrm{BH}}-\sigma_{\mathrm{e}}$ correlation. Finally, the high HST spatial resolution allows us to constrain the size of any cluster of dark objects alternative to a BH to $r_{\bullet}<00^{\prime} 035(\simeq 0.6 \mathrm{pc})$. Thus Centaurus A ranks among the best cases for supermassive Black Holes in galactic nuclei.

Key words. black hole physics - line: profiles - galaxies: individual: NGC 5128 - galaxies: kinematics and dynamics - galaxies: nuclei

^ Based on observations obtained at the Space Telescope Science Institute, which is operated by the Association of Universities for Research in Astronomy, Incorporated, under NASA contract NAS 526555 (HST Program ID 8119). Also based on observations collected at the European Southern Observatory, Paranal, Chile (ESO Program ID 63.P-0271A). 


\section{Introduction}

One of the drivers of current astrophysical research is understanding the birth and evolution of galaxies but it is becoming increasingly clear that the central supermassive black hole plays a major role in the evolution of the host galaxy.

During the last few years it has been realized that most, if not all, nearby luminous galaxies host a supermassive black hole $(\mathrm{BH})$ in their nuclei with masses in the $10^{6}-10^{10} M_{\odot}$ range (e.g. Ferrarese \& Ford 2005, and references therein). The BH mass $\left(M_{\mathrm{BH}}\right)$ is closely related with mass or luminosity of the host spheroid ( $L_{\mathrm{sph}}$, e.g. Kormendy \& Richstone 1995; Marconi \& Hunt 2003; Häring \& Rix 2004) and with the stellar velocity dispersion $(\sigma$, Ferrarese \& Merritt 2000; Gebhardt et al. 2000). These facts indicate that the formation of a massive $\mathrm{BH}$ is an essential ingredient in the process of galaxy formation and that there is a tight relation between host galaxy and the central BH. Like for the X-ray background, BHs are relics of past AGN activity (Yu \& Tremaine 2002; Marconi et al. 2004), and it is being realized that an accreting supermassive $\mathrm{BH}$ has a major impact on the star formation rate in the host galaxy: the feedback from the accreting BH (i.e. from the AGN) is thus responsible for setting the close relations between $\mathrm{BH}$ and host galaxy properties (e.g. Menci et al. 2003; Granato et al. 2004; Di Matteo et al. 2005).

BHs are detected and their mass directly measured in nearby galaxies using gas or stars as tracers of the kinematics in the nuclear region. The gas kinematical method has the advantage of being relatively simple, of requiring relatively short observation times and being applicable to AGNs. On the contrary, non gravitational or non circular motions might bias or completely invalidate the method. Moreover, detectable emission lines are not always present in galactic nuclei. The stellar dynamical method has the advantage that star motions are always gravitational and that stars are present in all galactic nuclei. However, long observation times are required to obtain high quality observations and stellar dynamical models are very complex leading to potential indeterminacy (Valluri et al. 2004; Cretton \& Emsellem 2004).

The weakest points of both methods - assumption of circular gravitational motions for gas kinematics, complexity of modeling for stellar dynamics - have shed some doubts on the reliability of $\mathrm{BH}$ mass estimates (e.g. Tremaine et al. 2002; Cappellari et al. 2002; Verdoes Kleijn et al. 2002; Valluri et al. 2004; Cretton \& Emsellem 2004). This is a very important issue because the correlations between $\mathrm{BH}$ mass and host galaxies properties, which are based mostly on $M_{\mathrm{BH}}$ from gas kinematic and stellar dynamics, are pivotal in understanding the coevolution of galaxies and BHs over cosmic time. Securing the correlations means establishing without any doubt the reliability of gas kinematics and stellar dynamics $M_{\mathrm{BH}}$ measurements.

Another important open issue is that the so-called supermassive BHs are in reality massive dark objects, i.e. clumps of dark mass unresolved at the spatial resolution of current observations. Only in the case of our own galaxy, we can safely state that the massive dark object is a supermassive black hole. In all other cases the massive dark object could very well be a cluster of dark stellar remnants (stellar mass black holes, neutron stars, hot jupiters etc.). Such clusters, due to friction and collisions, are doomed to collapse to a supermassive $\mathrm{BH}$ but timescales can be longer than the age of the universe (Maoz 1998). In order to exclude that a massive dark object is a cluster of dark objects one needs to constrain its size such that its life time is much shorter than the age of the universe, making a BH a very likely alternative (e.g. van der Marel et al. 1997; Macchetto et al. 1997; Schödel et al. 2003).

Centaurus A (NGC5128) is a giant elliptical galaxy and hosts a powerful radio source and an AGN revealed by the presence of a powerful radio and X-ray jet (see Israel 1998, for a review; see also Tingay et al. 2001; Grandi et al. 2003; Hardcastle et al. 2003; Evans et al. 2004, and references therein). The AGN in Centaurus A is low luminosity and is consistent with a misaligned BL-Lac (Capetti et al. 2000; Chiaberge et al. 2001). The most striking feature of Centaurus A is the dust lane, rich in molecular and ionized gas (Quillen et al. 1993, 1992; Leeuw et al. 2002; Karovska et al. 2003; Mirabel et al. 1999), which crosses the whole galaxy hiding the nuclear region under at least 7 mag of extinction in $V$ (Schreier et al. 1996; Marconi et al. 2000). A recent estimate of the distance of Centaurus A places the galaxy at $\sim 3.8 \mathrm{Mpc}$ (Rejkuba 2004) but in this paper we adopt $D=3.5 \mathrm{Mpc}\left(1^{\prime \prime} \simeq 17 \mathrm{pc}\right.$, Hui et al. 1993 ; Soria et al. 1996) to be consistent with our previous papers.

Schreier et al. (1998) found evidence for a disk-like feature in ionized gas disk from $\mathrm{Pa} \alpha$ HST/NICMOS images. Marconi et al. (2001) used near infrared spectroscopy to perform a gas kinematical analysis of such feature finding evidence for a $\mathrm{BH}$ with mass $M_{\mathrm{BH}}=2_{-1.4}^{+3.0} \times 10^{8} M_{\odot}$. The large error associated with $M_{\mathrm{BH}}$ takes into account the degeneracy due to the unknown inclination of the gas disk for which Marconi et al. (2001) could only assume $i>15 \mathrm{deg}$. Observations were performed with a spatial resolution of about $0.5(\simeq 8.5 \mathrm{pc})$. Even at that moderate spatial resolution the $\mathrm{BH}$ sphere of influence is well resolved: with $\sigma=138 \mathrm{~km} \mathrm{~s}^{-1}$ (Silge et al. 2005) the radius of the $\mathrm{BH}$ sphere of influence is $r_{\mathrm{BH}}=G M_{\mathrm{BH}} / \sigma^{2}=1.3^{\prime \prime}$. Centaurus $\mathrm{A}$ is thus the perfect benchmark to assess the validity of the gas kinematical method because the results will be little affected by spatial resolution or by uncertainties on the stellar contribution to the gravitational potential. The HST/STIS observations presented here have a spatial resolution which is a factor of 5 better than our previous ground-based observations allowing a unique test on the reliability of the gas kinematical method. A comparison between rotation curves taken at ground based and HST resolution was performed in Cygnus A and showed that after allowing for the different instrumental setups and spatial resolution, the best fit ground based model could nicely reproduce the observed STIS rotation curves (Tadhunter et al. 2003).

Recently Silge et al. (2005) have presented a stellar dynamical measurement of the BH mass in Centaurus A. To our knowledge this is the first time that a $\mathrm{BH}$ mass can be reliably measured in an external galaxy both with gas kinematics and stellar dynamics and the agreement of the two measurements can greatly strengthen the reliability of both methods. Cappellari et al. (2002) presented gas and stellar dynamical measurements of the BH mass in IC 1459 finding a 

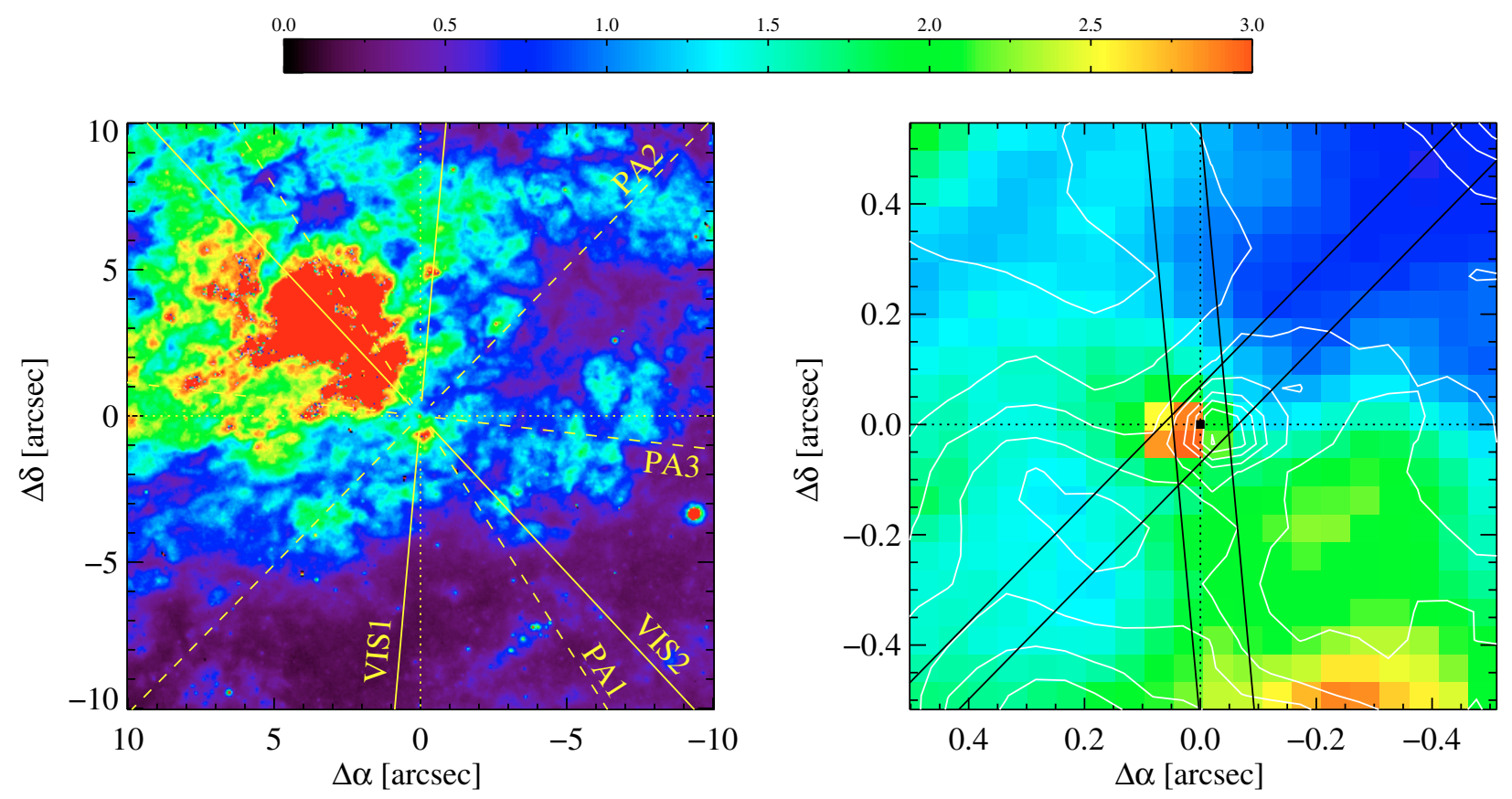

Fig. 1. Left: slit positions overlaid on the Centaurus A STIS image. VIS1 and VIS2 identify the STIS slit positions and PA1, PA2 and PA3 the ISAAC ones. The 0,0 position is the target position assumed by STIS and derived by the location of the bright star on the right (located at $\left.\simeq-9^{\prime \prime},-3^{\prime \prime}\right)$. Right: zoom on the central $1^{\prime \prime} \times 1^{\prime \prime}$.

disagreement of almost a factor 10 between the two. However in IC 1459 the rotation curve of the gas is not consistent with that of a rotating disk and the $M_{\mathrm{BH}}$ measurement cannot be considered reliable.

Last, but not least, the proximity of Centaurus A, combined with HST resolution will allow us to put a tight constraint on the size of any nuclear dark cluster with the possibility of establishing whether the putative $\mathrm{BH}$ is really a BH and not a cluster of dark stellar remnants.

In summary, Centaurus A is an ideal benchmark for direct BH mass measurements due to (i) its proximity; (ii) the wellresolved BH sphere of influence; (iii) the availability of data at different spatial resolution both of which resolve the BH sphere of influence and (iv) the availability of both gas and stellar dynamical $M_{\mathrm{BH}}$ measurement.

In Sect. 2 we present the HST/STIS observations and data reduction, and the re-analysis of our old VLT/ISAAC data. In Sect. 3 we describe the kinematical analysis, including the use of the Hermite expansion of the line profiles, and we present the kinematical parameters measured from all data. In Sect. 4 we describe the modeling of the kinematical data and how it is possible to obtain $M_{\mathrm{BH}}$ measurements from STIS and ISAAC data independently. In Sect. 5 we discuss the results from the modeling of STIS and ISAAC data, we evaluate the influence of the intrinsic surface brightness distribution of the disk on the derived $M_{\mathrm{BH}}$ value, and we present the problems which might be encountered when comparing observed and model velocity dispersions of the gas, the commonly adopted test of the reliability of the gas kinematical analysis. We compare our $M_{\mathrm{BH}}$ value with the stellar dynamical measurement, we discuss $M_{\mathrm{BH}}$ within the context of the correlation with host galaxy parameters and we then estimate an upper limit on the size of any dark cluster alternative to a BH. Finally, in Sect. 6 we summarize our work and present our conclusions.

\section{Observations and data reduction}

\subsection{STIS observations}

Centaurus A was observed with STIS on the HST in 2000 May 9 and July 1 . The nucleus location in Centaurus A is not well defined in optical $(<1 \mu \mathrm{m})$ images thus telescope acquisition was performed on a foreground star $\sim 10^{\prime \prime}$ away from the galaxy nucleus in order to register the coordinate system of the telescope $\left(\Delta \alpha, \Delta \delta=-9.3^{\prime \prime},-3.3^{\prime \prime}\right.$ in Fig. 1a). During each visit, a $\sim 5^{\prime \prime} \times 5^{\prime \prime}$ acquisition image was obtained with the F28X50LP filter on the foreground star which was subsequently centered and re-imaged following the ACQ and $\mathrm{ACQ} / \mathrm{PEAK}$ procedures. The exposure times of the acquisition images were 3 and $10 \mathrm{~s}$ respectively. After registering the coordinates system on the star, the telescope was subsequently pointed on the expected position of the Centaurus A nucleus derived from the analysis of WFPC2 images performed by Marconi et al. (2000). The observational strategy then consisted in obtaining an image in the F28X50LP filter to check the telescope pointing and slit locations with respect to the nucleus. Spectra were then obtained with the 52X0.1 aperture ( 0 ' $^{\prime} 1$ slit width) centered on the expected nucleus position. Given the different roll angle of the telescope during the two visits, the slits were located at PA $-5 \mathrm{deg}$ (hereafter VIS1) and $43 \mathrm{deg}$ (hereafter VIS2). The slit positions are overlaid on the STIS image in Fig. 1. At each slit position we obtained three spectra with the G750L grating centered at $7751 \AA$, in order to observe the [S III] $\lambda \lambda 9071,9533 \AA$ doublet. The second and third 
Table 1. Log of observations.

\begin{tabular}{lccccc}
\hline \hline ID & Date & Instr. & $\begin{array}{c}\text { PA } \\
{[\mathrm{deg}]}\end{array}$ & $\begin{array}{c}\mathcal{R}^{a} \\
\mathrm{Res}^{b} \\
{\left[{ }^{\prime}\right]}\end{array}$ \\
\hline VIS1 & May 9, 2000 & STIS & -5.0 & 1000 & 0.1 \\
VIS2 & July 1, 2000 & STIS & 43.0 & 1000 & 0.1 \\
PA1 & July 21, 1999 & ISAAC & 32.5 & 10000 & 0.5 \\
PA2 & July 22, 1999 & ISAAC & -44.5 & 10000 & 0.5 \\
PA3 & July 22, 1999 & ISAAC & 83.5 & 10000 & 0.5 \\
\hline
\end{tabular}

${ }^{a}$ Spectral resolution.

${ }^{b}$ Spatial resolution: FWHM of Point Spread Function (PSF) or seeing.

spectra were shifted along the slit by 0.5 and $1^{\prime \prime}$ in order to remove cosmic-ray hits and hot pixels. The spectra were obtained with the $0 . ' 1$ slit and no binning of the detector pixels, yielding a spatial scale of 0 .'0507/pix along the slit, a dispersion per pixel of $\Delta \lambda=4.882 \AA$ and a spectral resolution of $\mathcal{R}=\lambda /(2 \Delta \lambda) \simeq 1000$ at $9500 \AA$. The log of the observations is shown in Table 1 . The raw STIS images were processed with the calstis pipeline (Kim Quijano et al. 2003) using the best reference files available as of December 2003. The raw STIS spectra were processed with standard pipeline tasks but following a different procedure to correct the fringing exhibited by the STIS CCD in the far red $(>7500 \AA)$. In order to correct for fringing, we obtained contemporaneous flat fields with the same setup as the scientific observations. We then followed the prescriptions of Goudfrooij \& Christensen (1998). The onorbit flats were normalized using the task normspflat in the stsdas.hst_calib.stis package of IRAF ${ }^{1}$. Spectra were first prepared with prepspec and defringed with defringe. We did not use the task mkfringeflat to rescale and shift the flat fringes to those observed in the object because the signalto-noise ratio on the continuum was very poor. However, using two flats obtained in different spacecraft orbits during each visit, we verified that variations of the fringe positions, if any, do not affect the final rotation curves. The defringed spectra were then wavelength calibrated (wavecal) and corrected for $2 \mathrm{D}$ distorsion $(\mathrm{x} 2 \mathrm{~d})$. The spectra taken at different slit positions were then raligned using as reference the [S III] line and combined rejecting deviant pixels. The expected accuracy of the wavelength calibration is $0.1-0.3$ pix within a single exposure and 0.2-0.5 pix among different exposures (Kim Quijano et al. 2003) which converts into $\sim 15-45 \mathrm{~km} \mathrm{~s}^{-1}$ (relative) and $\sim 30-70 \mathrm{~km} \mathrm{~s}^{-1}$ (absolute). The relative error on the wavelength calibration is negligible for the data presented here because our analysis is restricted to the small detector region including [S III] $(\Delta \lambda<300 \AA)$. From an analysis of the arc exposures it is found that the lamp lines at $\sim 9000-9500 \AA$ have a $F W H M$ of $\sim 2.2$ pix consistently with the expected spectral resolution (Kim Quijano et al. 2003). Therefore, the degradation of the spectral resolution at $\sim 9000-9500 \AA$ produced by the grating corresponds to a velocity dispersion of $\sigma=140 \mathrm{~km} \mathrm{~s}^{-1}$. When also the degradation introduced by the slit width

${ }^{1}$ IRAF is distributed by the National Optical Astronomy Observatories, which are operated by the Association of Universities for Research in Astronomy, Inc., under cooperative agreement with the National Science Foundation. is considered, the final instrumental resolution corresponds to $\sigma=160 \mathrm{~km} \mathrm{~s}^{-1}$, i.e. the width of the arc lines at 9000-9500 А.

The target acquisition strategy followed in these observations ensures that the STIS slits are centered on the same point, regardless of its distance from the Centaurus A nucleus. This can be verified from the images obtained just after the telescope offset from the acquisition star and before the spectra. The aperture center coordinates on the images are given by the HOSIAX and HOSIAY keywords. We used these nominal aperture centers as pivots for rotation and realignment of the images taken in the 2 different visits. By using 9 point sources common to both images we then verified that the images are aligned to better than 0.1 and 0.25 pixels rms in RA and Dec, i.e. 0.'006 and $0{ }^{\prime} 014$ respectively. This is then the accuracy with which the STIS slits are centered on the same point which is shown as a filled square in Fig. 1b.

\subsection{ISAAC observations}

We complement the new HST STIS Observations with the $J$-band, ground-based spectroscopic data already presented and analyzed by Marconi et al. (2001). Briefly, the data were obtained in 1999 using ISAAC at the ESO VLT and consist of medium resolution spectra obtained with the 0.3 slit and the grating centered at $\lambda=1.27 \mu \mathrm{m}$. The dispersion is $0.6 \AA /$ pix, yelding a resolving power of 10000 . Seeing during the observations was in the range 0. '4-0.' 6 , with photometric conditions. The observation procedure was to obtain an acquisition image in the $K$ band and then center the slit on the prominent $K$ band peak in the nuclear region of Centaurus A. Spectra were then obtained with the slits placed at three different position angles, summarized in Table 1. More details on observations and data reduction can be found in Marconi et al. (2001). We have re-reduced the ISAAC spectra following the same strategy as Marconi et al. (2001) but with a difference: before coadding all the wavelength calibrated frames, we have traced the position of the nuclear continuum peak along the dispersion direction and used this trace to rectify the dispersion direction. This ensures that there is no variation of the location of the continuum peak along the slit in the wavelength range covered by the observations.

\section{Results}

\subsection{STIS line kinematics}

Figure 2 shows the average STIS spectrum from VIS1 and VIS2 extracted from a $0 !^{\prime} 1 \times 0.0^{\prime} 6$ aperture centered on the assumed nucleus position. The lines $\mathrm{H} \alpha$, [N II], [S II], [O II], [S III] and [C I] are clearly detected and their relative strenght are an indication of the strong reddening which affects the nuclear region of Centaurus A $\left(A_{\mathrm{V}}>7\right.$ mag e.g., Schreier et al. 1996; Marconi et al. 2000): typically, $\mathrm{H} \alpha+[\mathrm{N} \mathrm{II}]$ is stronger than $[\mathrm{S} \mathrm{III}]$ while here $[\mathrm{S} \mathrm{III}] / \mathrm{H} \alpha+[\mathrm{N} \mathrm{II}]>10$. Therefore, only [S III] $\lambda 9500 \AA$ has enough signal-to-noise ratio (hereafter SNR) for kinematical analysis. We thus focus only on the 9300-9800 ̊̊ spectral region. 


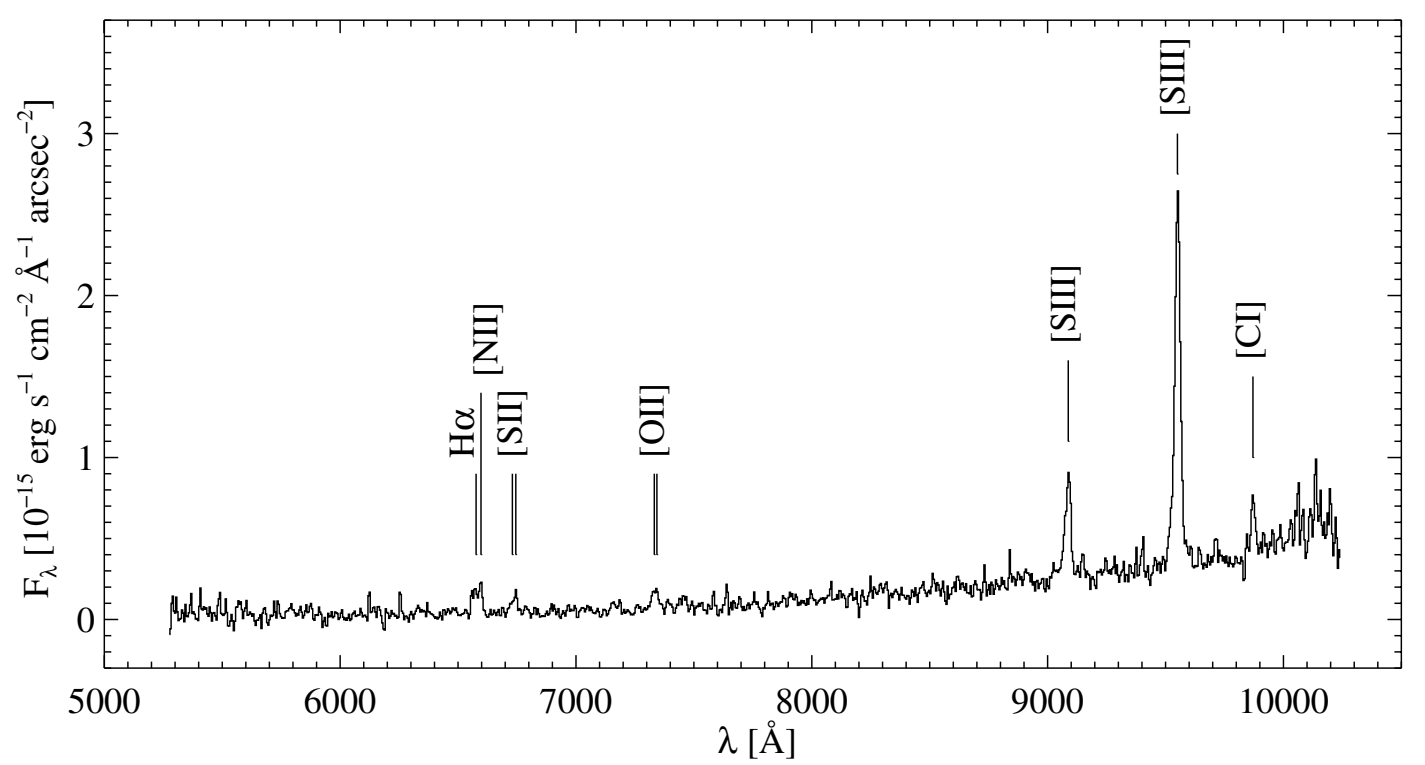

Fig. 2. Average STIS spectrum from VIS1 and VIS2 extracted from a $0{ }^{\prime} 1 \times 00^{\prime} 6$ aperture centered on the assumed nucleus position.

After selecting the spectral region, we subtracted the continuum by fitting a linear polynomial row by row along the dispersion direction. The continuum subtracted lines were then fitted row by row with Gaussian functions using the task specfit in the IRAF stsdas.contrib package. When the signalto-noise ratio (SNR) was insufficient (faint line) the fitting was improved by co-adding two or more pixels along the slit direction.

Fitting single Gaussians is acceptable for most of the positions along the slit but it does not produce good results for the points in the nuclear region where a careful analysis of the line profiles shows that they are persistently asymmetric with the presence of a blue wing (Fig. 3). A fit row by row with two Gaussian components, the main component and the blue one, shows that, within the large uncertainties, the "blue wing" has always the same velocity and width. We have thus deblended the "blue" component in the spectrum obtained by co-adding the central 7 rows. The velocity and width of the blue component $\left(v=-240 \mathrm{~km} \mathrm{~s}^{-1}\right.$ and $\sigma=360 \mathrm{~km} \mathrm{~s}^{-1}$ for VIS1, $v=-320 \mathrm{~km} \mathrm{~s}^{-1}$ and $\sigma=430 \mathrm{~km} \mathrm{~s}^{-1}$ for VIS2) were then held fixed in the row by row fit significantly improving the fitting of the line profiles (Fig. 3). A similar blue wing was detected and deblended in $\mathrm{H} \alpha+[\mathrm{N} \mathrm{II}]$ in NGC 4041 (Marconi et al. 2003a) and, as in that paper, the present data does not allow to identify the nature of the blue wing. We remark that the final rotation curve does not depend on the exact values of $v$ and $\sigma$ used to parameterize the blue wing since that component is faint compared to the main one. However, the rotation curves obtained without taking into account the blue wing are not consistent with a circularly rotating disk, like in the case of NGC 4041 (Marconi et al. 2003a).

The fitting procedure provides line-of-sight velocities, velocity dispersions and surface brightnesses along each slit for the [S III] $\lambda 9500 \AA$ A emission line which are shown in Fig. 4.

The observed line surface brightness in VIS1 and VIS2 are strongly peaked at the expected nucleus positions, similarly to what was observed in $\mathrm{Pa} \alpha$ (Schreier et al. 1998) and [Fe II] (Marconi et al. 2000, 2001). The SNR of the data does not allow the detection of extended emission beyond $1^{\prime \prime}$ from the nucleus. The rotation curve in VIS1 is "S-shaped" as expected in the case of a circularly rotating disk observed with a slit close to its major axis. In VIS2 the S-shaped pattern seems superimposed on a constant velocity gradient. The amplitude of the rotation curves is $\sim 300 \mathrm{~km} \mathrm{~s}^{-1}$ (VIS1) and $\sim 200 \mathrm{~km} \mathrm{~s}^{-1}$ (VIS2) suggesting that the VIS1 slit is closer to the orientation of the disk line of nodes. The behaviour of the velocity dispersion is similar to what is qualitatively expected from a rotating disk, i.e. a constant $\sigma$ which increases toward the nucleus location reaching a peak value of $\sim 350 \mathrm{~km} \mathrm{~s}^{-1}$.

\subsection{ISAAC line kinematics}

The fit with one or two Gaussians - in case of a significant blue wing - produces good results for the STIS data because of the moderately low SNR and spectral resolution $(\sim 1000)$. However, the ISAAC data have a much higher SNR and spectral resolution and the line profiles present a significant structure in the nuclear region similar to the expected one in case of unresolved rotation. Marconi et al. (2001) estimated kinematical parameters by fitting single Gaussians to [Fe II] and $\mathrm{Pa} \beta$ lines. While the fitting was acceptable for the purposes of that paper, it had significant residuals. In order to estimate accurately the average velocity and velocity dispersion, i.e. the same parameters computed by our model (see below), the residuals should not have any structure. When a single Gaussian is a too simple model for the observed line profile, a possibility is to fit 2 Gaussians, but the fitting is unstable and Gaussian parameters are strongly correlated. Therefore we decided to model the line profile with the expansion in Hermite polynomial introduced by van der Marel \& Franx (1993) coupled with the penalized pixel fitting technique (Merritt 1997; Cappellari \& Emsellem 2004). Such parameterization of 

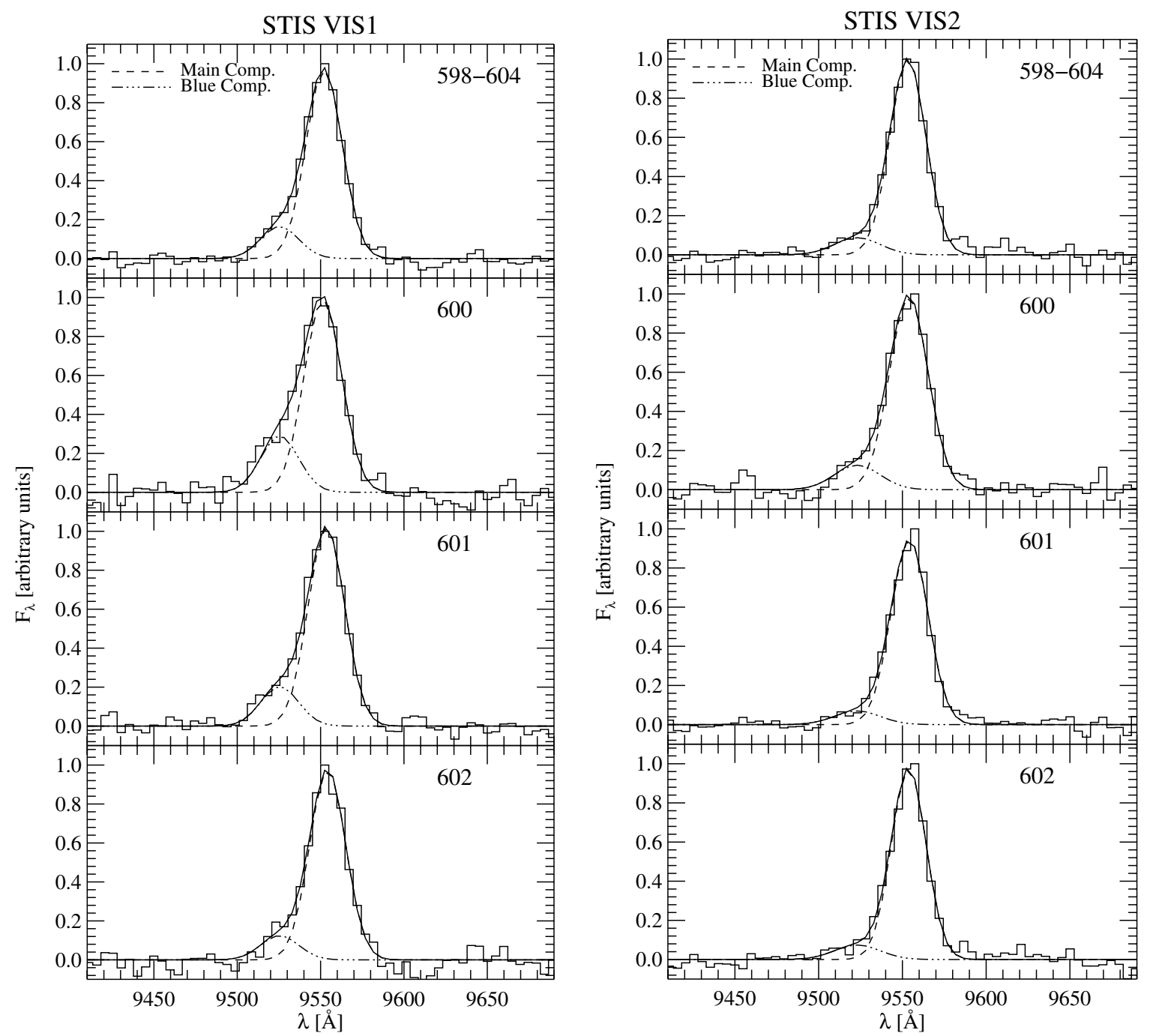

Fig. 3. Examples of the fits of the line profiles for the STIS observations of VIS1 and VIS2. The dashed and dot-dashed lines identify the two components of the fit, called "main" and "blue". For every slit position the blue component has the same velocity and width at each row and their values are determined in the fit of the overall nuclear spectrum shown in top panels. The numbers in the upper right corners of each panel are the row and range of rows where the fit was performed.

the line profile coupled with the penalized fitting technique has been used to describe the Line of Sight Velocity distribution of stellar kinematical data but it is easily applicable to the analysis of gas kinematical data. Of course, this refinement is necessary only because of the signal to noise and spectral resolution of the data is moderately high, otherwise a single Gaussian would be more than adequate to describe the data. Briefly, following van der Marel \& Franx (1993) we parameterize the line profile $L(v)$ as:

$L(v)=\gamma \alpha(w)\left[1+h_{3} H_{3}(w)+h_{4} H_{4}(w)\right]$

where

$w=\frac{v-v_{0}}{\sigma_{0}}$

$\alpha(w)=\frac{1}{2 \pi} \mathrm{e}^{-\frac{1}{2} w^{2}}$

and $H_{3}(w), H_{4}(w)$ are the third and fourth order Hermite polynomials with the normalization adopted by van der Marel \& Franx (1993). $v_{0}$ and $\sigma_{0}$ are the central velocity and velocity dispersion of the Gaussian component and correspond to the average velocity and velocity dispersion of the line profile when it is a perfect Gaussian. $h_{3}$ and $h_{4}$ quantify the deviations from the Gaussian line profile and $h_{3}=h_{4}=0$ corresponds to the perfect Gaussian case. Though the Hermite expansion can be extended to orders higher than 4th, we did not find significant improvements in the profile fitting, given the SNR of the data. The above line profile was fitted by minimizing the penalized $\chi^{2}$ where

$\chi_{\mathrm{p}}^{2}=\chi^{2}\left[1+\beta^{2}\left(h_{3}^{2}+h_{4}^{2}\right)\right]$

see Cappellari \& Emsellem (2004) for more details. $\chi^{2}$ is the classical Chi-squared and $\beta$ is a "bias" factor which favours best fits with low $h_{3}$ and $h_{4}$ values. We found that $\beta=1$ is adequate to the spectral resolution and SNR of our data. We investigated comprehensively the possibility that lines did not share common kinematics but we did not find evidence for this. On this basis we proceded in the subsequent analysis assuming 

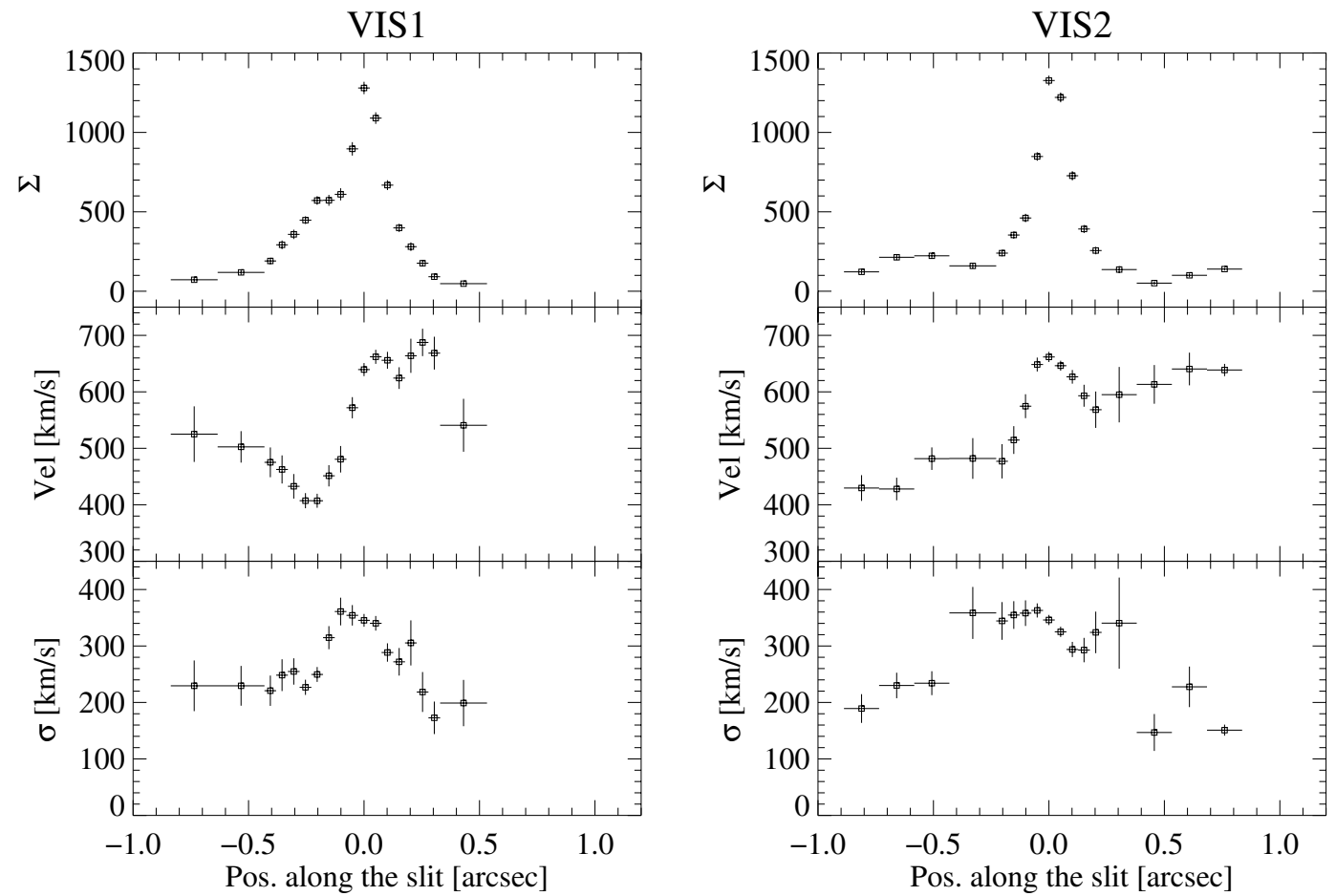

Fig. 4. From top to bottom: average emission line surface brightnesses (units of $10^{-16} \mathrm{erg} \mathrm{s}^{-1} \mathrm{~cm}^{-2} \operatorname{arcsec}^{-2}$ ), velocities, and velocity dispersion observed along the slit in STIS observations. Columns 1 to and 2 refers to VIS1 and VIS2, respectively.
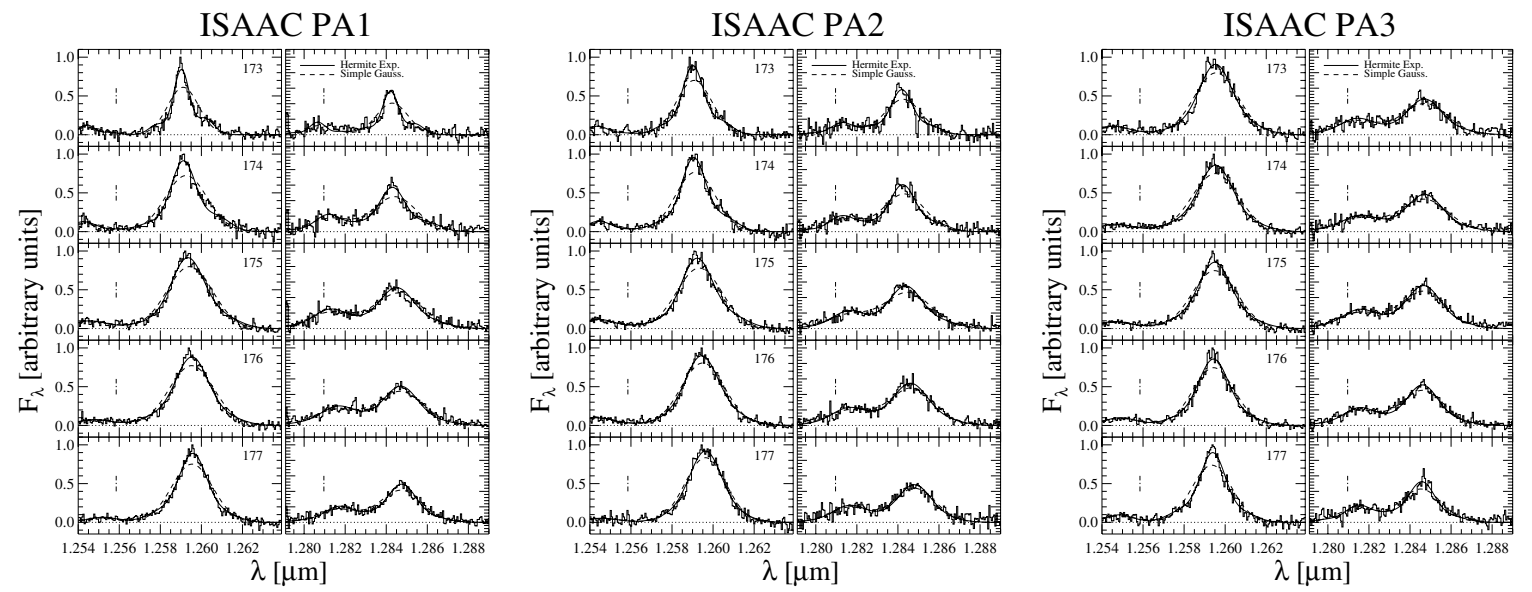

Fig. 5. Examples of the fits of the line profiles for the ISAAC observations of PA1, PA2 and PA3. The solid lines represents the case of the Hermite expansion while the dashed line is the simple Gaussian fit. The line at shorter wavelengths is [Fe II] while at longer wavelengths the strongest line is $\mathrm{Pa} \beta$. All emission lines have the same average velocity, velocity dispersion and Hermite coefficients. At the blue side of [Fe II] a faint line is visible, this is [S IX]. The line at the blue side of $\mathrm{Pa} \beta$ is another [Fe II]. The dot dash short line mark the expected position of the blue component found in the [S III] line.

that all lines in the spectrum have the same average velocity, velocity dispersion, $h_{3}$ and $h_{4}$.

Examples of the fits of the line profiles for the ISAAC observations of PA1, PA2 and PA3 are shown in Fig. 5 where we also compare the Hermite expansion with the case of the pure Gaussian fit (dashed line).

The fitting procedure provides line-of-sight velocities, velocity dispersions and surface brightnesses along each slit for the $[\mathrm{Fe} \mathrm{II}]$ and $\mathrm{Pa} \beta$ emission lines which are shown in Fig. 6 . In Fig. 7 we compare our new determination of the average velocity with that by Marconi et al. (2001). The new rotation curves do not differ significantly from the old ones apart from being less noisy. ISAAC kinematical data are described and discussed at length in Marconi et al. (2001) and we refer to that paper for more details. Here we concentrate exclusively in the nuclear region, i.e. $\leq 2^{\prime \prime}$ from the position of the $K$ band peak. In the nuclear region the ISAAC data exhibit the same behaviour as the STIS ones, i.e. strongly peaked surface brightness profiles, S-shaped rotation curves with amplitudes that vary with slit PA $\left(\sim 150 \mathrm{~km} \mathrm{~s}^{-1}\right.$ at PA1, $\sim 200 \mathrm{~km} \mathrm{~s}^{-1}$ at PA2, 

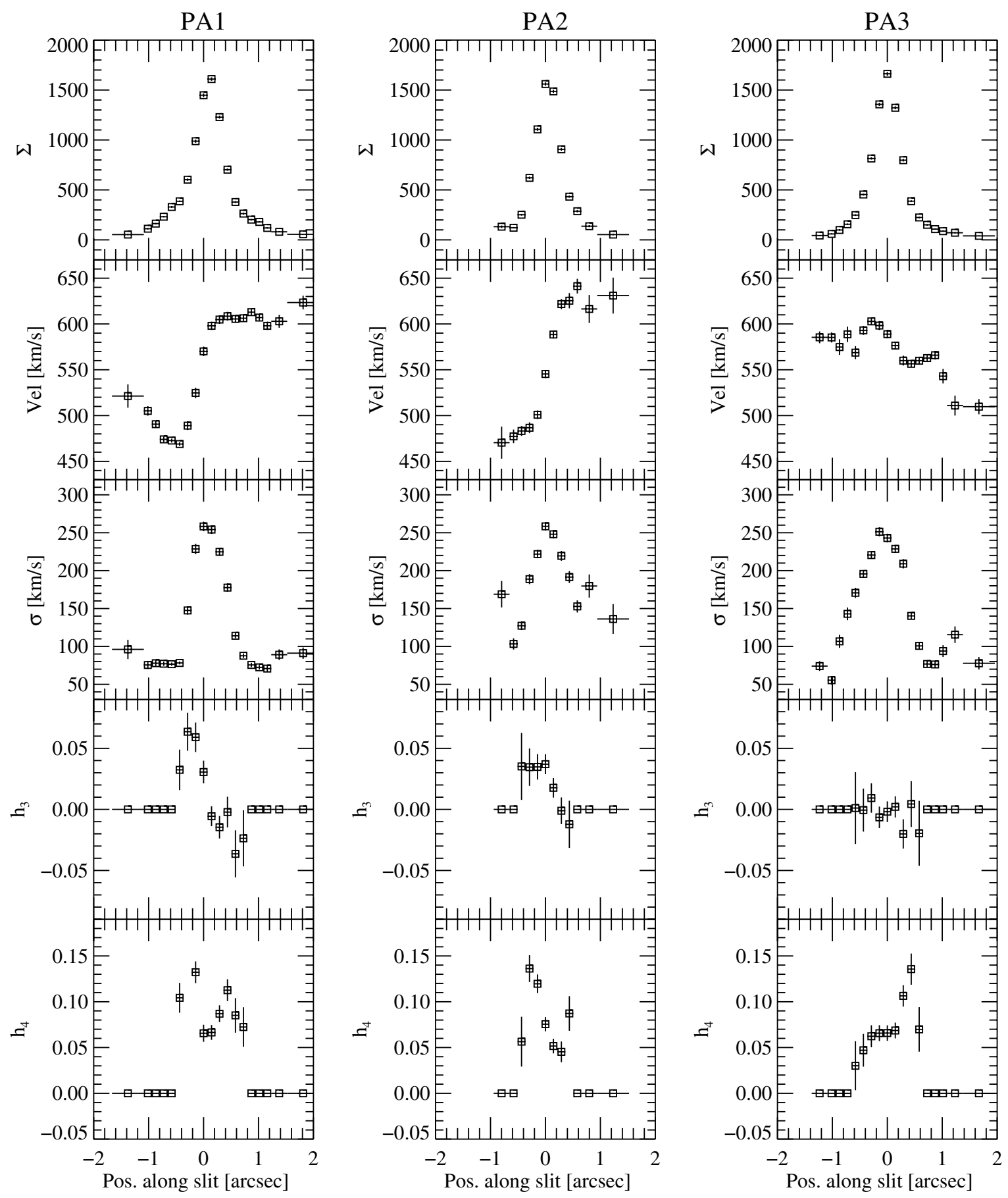

Fig. 6. From top to bottom: average emission line fluxes, velocities, velocity dispersion, $h_{3}$ and $h_{4}$ observed along the slit in ISAAC observations. Columns 1 to 3 refers to PA1, PA2 and PA3, respectively. The kinematical quantities were derived from a simultaneous fit of Pa $\beta$ and [Fe II].

and $\sim 50 \mathrm{~km} \mathrm{~s}^{-1}$ at PA3). The observed velocity dispersion is characterized by a well defined constant plateau, expecially in PA1, and a smoth increase toward the nucleus location where it reaches an amplitude of $\sim 250 \mathrm{~km} \mathrm{~s}^{-1}$ at all slit orientations. $h_{3}$ and $h_{4}$ are significantly different from 0 only in within $1^{\prime \prime}$ of the nucleus where the SNR of the data is higher. In all other locations they have been fixed to 0 .
In Fig. 8 we compare the rotation curve from STIS at VIS2 and the rotation curves from ISAAC at PA1. This comparison is not affected by geometrical effects because the two slits have a similar orientation, differing only by $5 \mathrm{deg}$. The ISAAC data are characterized by a much higher SNR and a by a smoother rotation curve. The effect of the different spatial resolution and slit width is clearly that of smoothing away the high velocity 


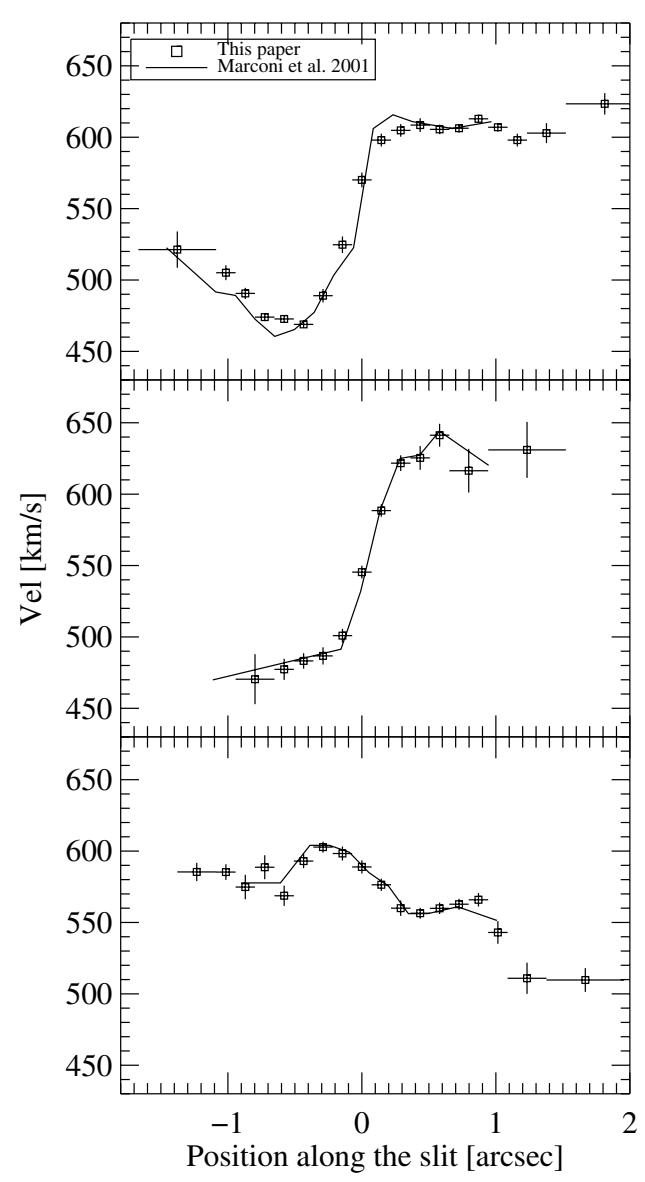

Fig. 7. Comparison of average velocities from [Fe II] and $\mathrm{Pa} \beta$ emission lines with those from Marconi et al. (2001).

increase observed in the STIS data. This effect is expected and was discussed at length by Macchetto et al. (1997). The STIS rotation curve is thus in good agreement with the ISAAC data apart for the two couples of points \pm 0 ' $^{\prime} 7$ away from the center. It is difficult to establish whether the discrepancy is due to low SNR of the data and/or to the presence of non circular motions in the [S III] emitting medium. Since we have verified that the inclusion of those points does not change the final results apart for incresing $\chi^{2}$, we have decided not to consider them in the following analysis.

\section{Modeling the rotation curves}

The aim of our new HST/STIS observations is to confirm at higher angular resolution the detection of the supermassive BH in Centaurus A by Marconi et al. (2001). Both STIS and ISAAC data are analized to verify if they provide consistent results.

To model the kinematical data we follow the procedure first described by Macchetto et al. (1997) and subsequently refined by several authors (van der Marel \& van den Bosch 1998; Marconi et al. 2001; Barth et al. 2001; Marconi et al. 2003a). Our modeling code, described in detail by Marconi et al. (2003a), was used to fit the observed rotation curves. Very briefly the code computes the rotation curves of the gas

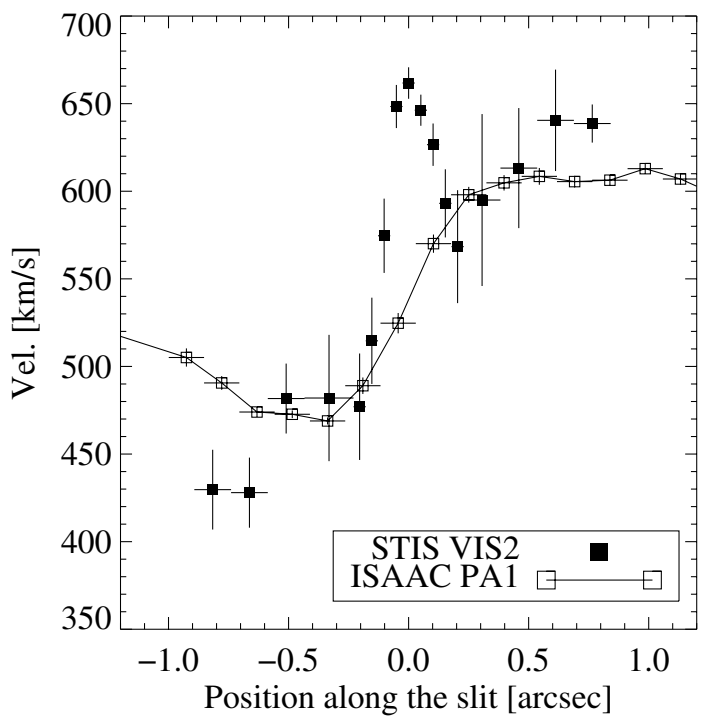

Fig. 8. Comparison of average velocities from STIS VIS2 and ISAAC PA1.

assuming that the gas is rotating in circular orbits within a thin disk in principal plane of the galaxy potential. We neglect any hydrodynamical effect like gas pressure. The gravitational potential is made of two components: the stellar potential, characterized by its mass-to-light ratio $M / L$, and a dark mass concentration (the putative black hole), spatially unresolved at HST+STIS resolution and characterized by its total mass $M_{\mathrm{BH}}$. In computing the rotation curves we take into account the finite spatial resolution of the observations, the intrinsic surface brightness distribution of the emission lines (hereafter ISBD) and we integrate over the slit and pixel area. The free parameters characterizing the best fitting model are found by standard $\chi^{2}$ minimization.

In Sect. 4.1 we determine the radial light profile of the stellar component which, multiplied, by $M / L$ directly provide the enclosed mass at a given distance from the nucleus. In Sect. 4.2 we determine the ISBD which is the weight for averaging the kinematical quantities. Finally, in Sect. 4.3 we present the results of the kinematical fitting obtained using the stellar mass distribution and intrinsic surface brightness previously determined. In the same section we also present a new way to compute the kinematical model quantities in order to avoid subsampling problems.

\subsection{The stellar mass distribution}

The inversion procedure to derive the stars distribution from the surface brightness is not unique if the gravitational potential does not have a spherical symmetry, revealed by circular isophotes. Assuming that the gravitational potential is an oblate spheroid, the inversion depends on the knowledge of the potential axial ratio $q$, and the inclination of its principal plane with respect to the line of sight, $i$. As these two quantities are related by the observed isophote ellipticity, we are left with the freedom of assuming different galaxy inclinations to the line of sight. Following van der Marel \& van den Bosch (1998), 

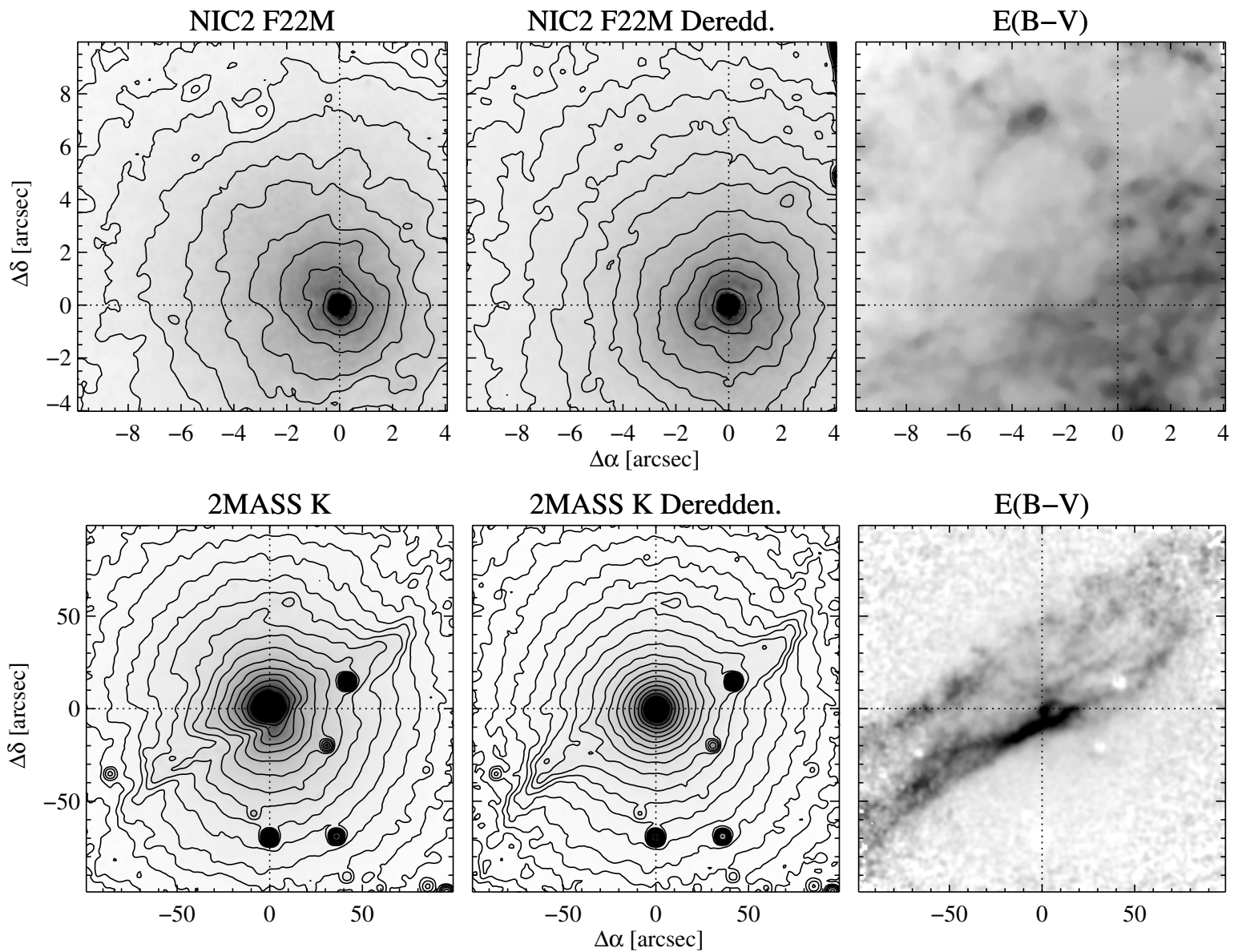

Fig. 9. Top, from left to right: observed NICMOS F222M image, dereddened F222M image, derived $E(B-V)$ map. Bottom, from left to right: observed 2MASS $K$ band image, dereddened $K$ image, derived $E(B-V)$ map.

we assumed an oblate spheroid density distribution parameterized as:

$\rho(m)=\rho_{0}\left(\frac{m}{r_{b}}\right)^{-\alpha}\left[1+\left(\frac{m}{r_{b}}\right)^{2}\right]^{-\beta}$

$m$ is given by $m^{2}=x^{2}+y^{2}+z^{2} / q^{2}$ where $x y z$ is a reference system with the $x y$ plane corresponding to the principal plane of the potential and $q$ is the intrinsic axial ratio. This threedimensional density model is converted to an observed surface brightness distribution in the plane of the sky by integrating along the line of sight, convolving with the Point Spread Function (PSF) of the telescope+instrument system and averaging over the detector pixel size. Then, the derived model light profile can be directly compared with observed ones. A detailed description of the relevant formulas and of the inversion and fit procedure is presented in the Marconi et al. (2003a).

We reconstructed the galaxy light profile combining a NICMOS F222M ( $K$ band) image obtained with the NIC2 camera (pixel size 0.'075; see Schreier et al. 1998; and Marconi et al. 2000, for details) with a 2MASS $K$ band image from the Large Galaxy Atlas (pizel size 1"; Jarrett et al. 2003). The first image, with smaller pixel size and better spatial resolution was used for the central regions $\left(r<6^{\prime \prime}\right)$ while for the more extended emission we took advantage of the larger field of view of the 2MASS image.

The nuclear region of Centaurus A is strongly reddened due to the well known dust lane. This effect can be seen also in the $K$ band and significantly affects the profile determination. Therefore we applied a reddening correction to the images, following Marconi et al. (2000) where the reader should refer to for more details. Briefly, using available NIC2 F160W and 2MASS $J$ band images of Centaurus A, we computed the $H-K$ and $J-K$ colors. Assuming as intrinsic colors $H-K=0.2$ and $J-K=0.92$ we can then estimate reddening using the extinction curve by Cardelli et al. (1989) with $A_{\mathrm{V}} / E(B-V)=3.1$. Figure 9 shows the effects of this reddening correction. The isophotes from the NIC2 F222M image prior to correction are elongated in a direction perpendicular to the dust lane, while after correction their circular simmetry is recovered. Similarly, in the 2MASS $K$ band image prior to correction one can clearly see the distorsion caused by the lower edge of the dust lane running approximately at PA $-45 \mathrm{deg}$. After correction the circular simmetry is well recovered in the central region. There is still a residual distortion due to an over correction in the region where the dust screen approximation fails. However this distortion can be easily taken into account in the ellipse fitting described below. 

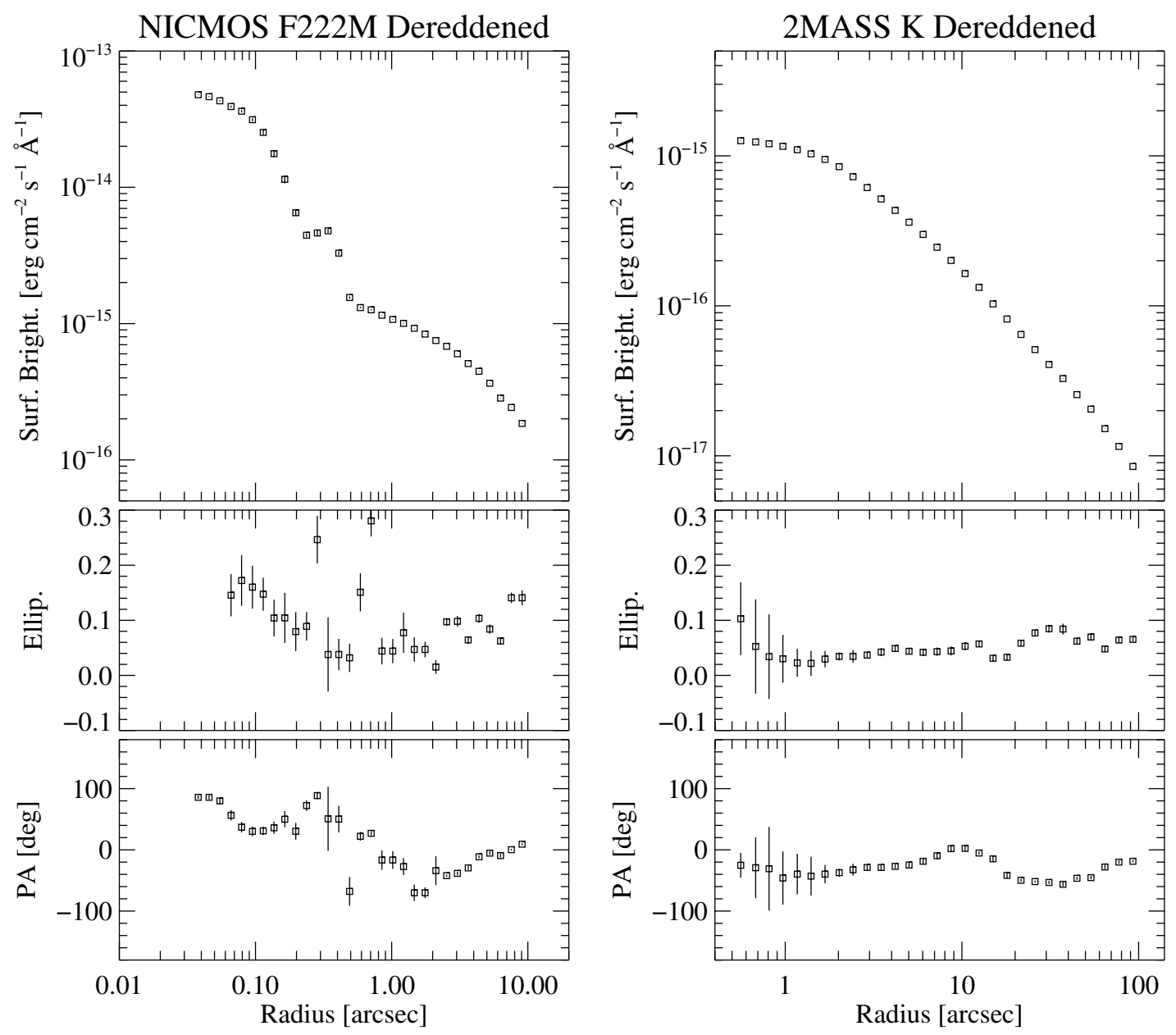

Fig. 10. Results of ellipse fitting on dereddened NICMOS F222M (left) and 2MASS K images (right). The top panels show the observed surface brightness as a function of radius. Middle panels and lower panels show ellipticity and position angle of the ellipse major axis respectively.

We used the IRAF/STSDAS program ellipse to fit elliptical isophotes to the galaxy after reddening correction and the results are shown in Fig. 10.

The surface brightness profiles derived from NICMOS data show a central unresolved source clearly identifiable by its first Airy ring at $\sim 0$ ' 3 . The ellipticity is small being $<0.1$ if one excludes the inner $0 . ' 4$ where emission is dominated by the central unresolved source. The position angle shows significant variations but it is probably affected by residual reddening. The surface brightness profile, ellipticity and PA show a smoother behaviour in the 2MASS data. The ellipticity is still small and increases to 0.1 only at scales larger than those probed by our spectra $\left(>20^{\prime \prime}\right)$.

As shown in Fig. 11 the 2 dereddened profiles are in excellent agreement in the overlap region taking into account that no rescaling of the data was performed.

In order to determine the stellar contribution to the mass density we can then safely assume a spherical simmetry since the isophotes, at least in the nuclear region, are close to circular. Moreover, we can use an observed light profile obtained by merging the NICMOS $\left(r \leq 6^{\prime \prime}\right)$ and the 2MASS ones $\left(r>6^{\prime \prime}\right)$. Since the spatial resolution of the observations affects only the inner parts of the light profiles, the PSF to be used in the fitting

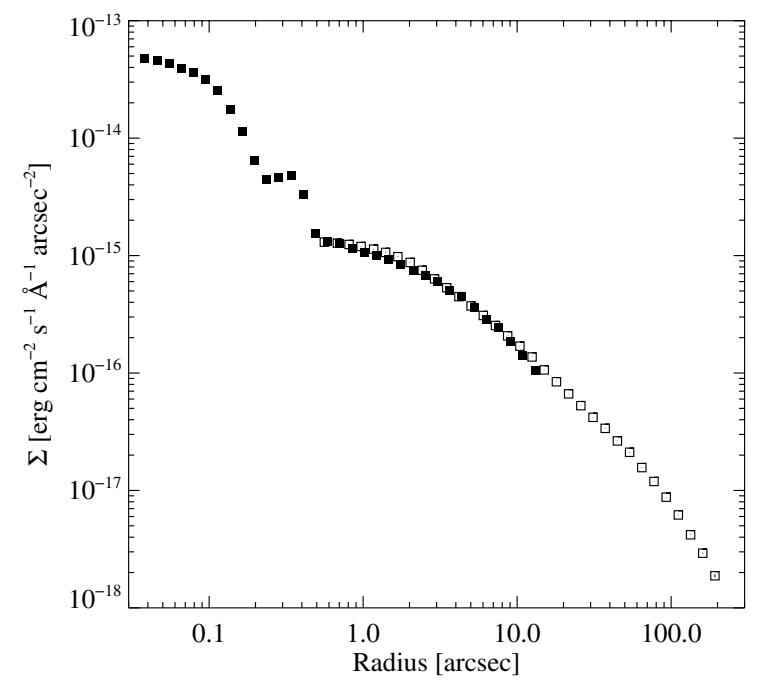

Fig. 11. Comparison of the surface brightness profiles obtained from reddening corrected NICMOS F222M (filled squares) and 2MASS $\mathrm{K}$ images (empty squares).

is the one of NIC2 F222M, which we take from TinyTim (Krist \& Hook 1999). 


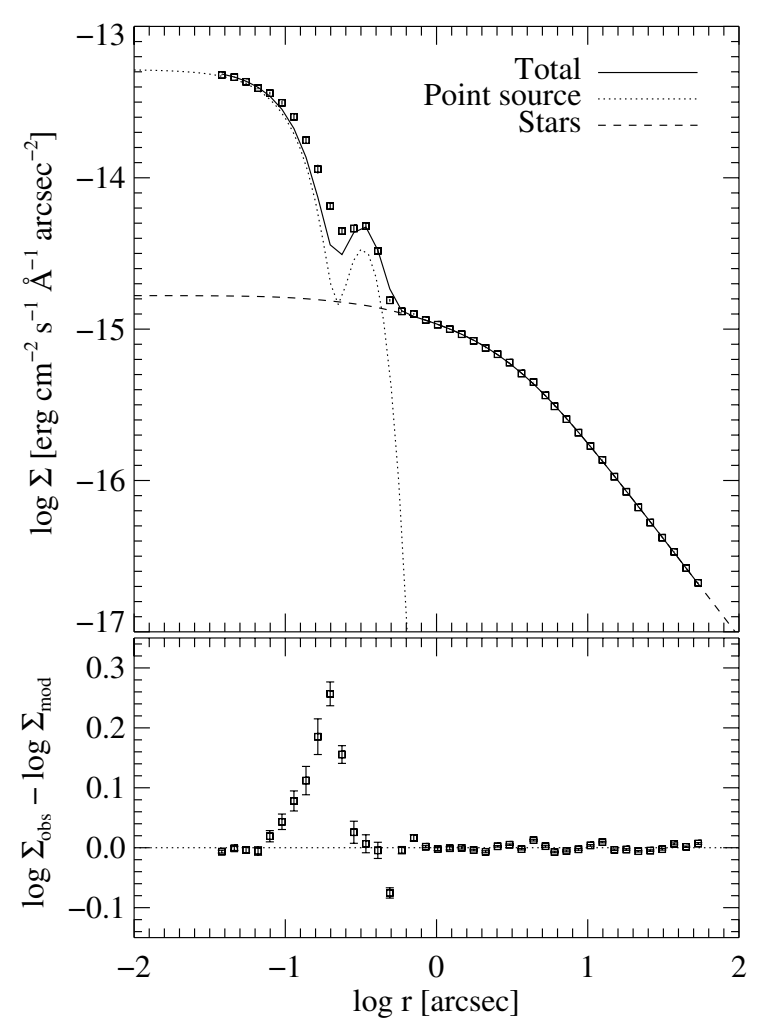

Fig. 12. Upper panel: brightness profile of Centaurus A in the $K$ band resulting from merging the NICMOS F222M and 2MASS K profiles. Surface brightness is in units of $\operatorname{erg~s}^{-1} \mathrm{~cm}^{-2} \AA^{-1} \operatorname{arcsec}^{-2}$. The solid line is the fit to the profile obtained from a spherical stellar density distribution with an added nuclear point source. The dotted and dashed lines show the relative contributions of point source and stellar density, respectively.

The best fit of the observed stellar light profile is shown in Fig. 12 and is characterized by $\rho_{0}=(1000 \pm 3) M_{\odot} \mathrm{pc}^{-3}$ (for $M / L=1), \alpha=0.81 \pm 0.01, \beta=0.74 \pm 0.01$ and $r_{b}=33^{\prime \prime} 62 \pm 0{ }^{\prime} 01$ ( $\sim 62 \mathrm{pc}$ at the distance of Centaurus A). The $1 \sigma$ statistical errors were estimated with 100 montecarlo evaluations of the fit. The presence of an unresolved nuclear point source, and its associated Airy ring, are clearly visible in Fig. 10. Indeed a point source with flux $(3.39 \pm 0.01) \times 10^{-15} \mathrm{erg} \mathrm{s}^{-1} \mathrm{~cm}^{-2} \AA^{-1}$ ( $K=10.1 \mathrm{mag}$ ) had to be added to the extended luminosity distribution to provide a good fit to the brightness profile. The fit is excellent everywhere except for large residuals in the region where the central unresolved emission dominates. This is not a serious issue since it is related to the detailed shape of the PSF, which is likely distorted by the reddening correction procedure described in Sect. 4.1, and does not affect the stellar emission. It has been shown by Quillen et al. (2001) that unresolved infrared sources are found in the great majority of HST NICMOS images of Seyfert galaxies and that their luminosities strongly correlate with both the hard X-ray and the [O III] line luminosity. This result suggests a dominant AGN contribution to the IR emission. Moreover, we found that the central unresolved source is significantly polarized $P=11.1 \%$ (Capetti et al. 2000) and this definitely excludes that it could be emission from an unresolved star cluster.

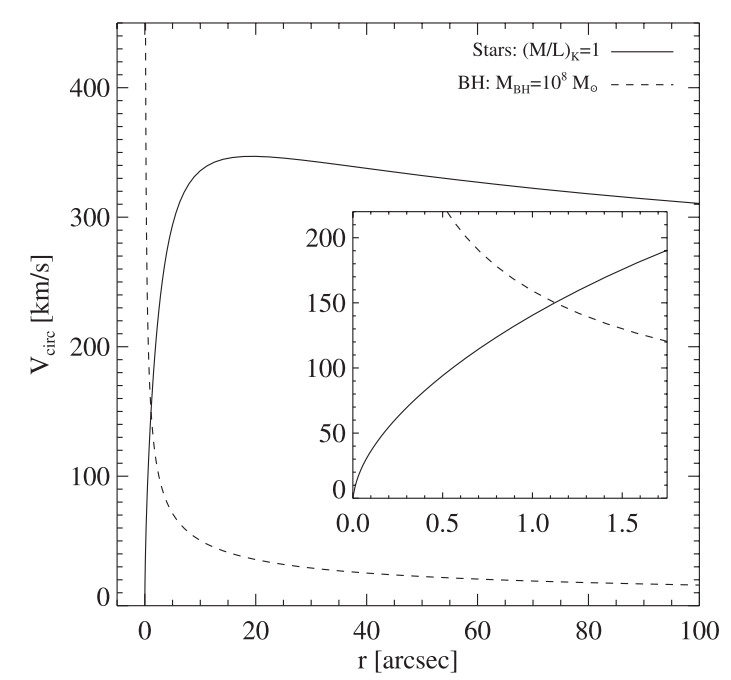

Fig. 13. Circular velocity derived from the model fit in Fig. 12 and computed assuming $M / L=1$ (solid line). The dashed line represents the rotation curve expected in the case of a $\mathrm{BH}$ with mass $M_{\mathrm{BH}}=10^{8} M_{\odot}$. The small inset shows the behaviour of the circular velocity in the nuclear region. The radius of the BH sphere of influence, i.e. the radius at which the contribution of stars and $\mathrm{BH}$ to the circular velocity are equal is $\simeq 1$." 1 .

Finally, in Fig. 13 we plot the circular velocity due to the stars as a function of the distance from the nucleus, computed assuming $M / L=1$ (solid line). The dashed line represents the circular velocity expected from a $\mathrm{BH}$ with mass $M_{\mathrm{BH}}=10^{8} M_{\odot}$. Below $1^{\prime \prime}$, the BH completely dominates the gravitational potential, a fact already noted by Marconi et al. (2001). At $r=1.1^{\prime \prime}$, the contribution of BH and stars to the gravitational potential are equal. This size can be compared with the standard definition of the radius of the $\mathrm{BH}$ sphere of influence, $r_{\mathrm{BH}}=G M_{\mathrm{BH}} / \sigma_{\mathrm{e}}^{2}$, where $G$ is the gravitational constant and $\sigma_{\mathrm{e}}$ is the effective velocity dispersion of the stars. In the case of Centaurus A, $\sigma_{\mathrm{e}}=138 \pm 10 \mathrm{~km} \mathrm{~s}^{-1}$ (Silge et al. 2005, see Sect. 5.5) which combined with $M_{\mathrm{BH}}=10^{8} M_{\odot}$ yelds $r_{\mathrm{BH}}=1.3 \pm 0.3^{\prime \prime}$ in excellent agreement with the estimate from our analysis of the stellar potential.

\subsection{The intrinsic line surface brightness distribution}

The observed kinematical quantities are averages over apertures defined by the slit width and the detector pixel size along the slit. The ISBD is thus a fundamental ingredient in the modeling of the kinematical quantities because it is the weight of the averaging process (see, e.g., the discussion and Appendix B in Marconi et al. 2003a). Barth et al. (2001) derived the ISBD by deconvolving a continuum subtracted emission line image and were successful in reproducing the microstructure of the rotation curves thus reducing the overall $\chi^{2}$ of their best fitting model. However, apart for the microstructure of the rotation curves which has little impact on the final $M_{\mathrm{BH}}$ estimate, Marconi et al. (2003a) showed that it is crucial to adopt a good ISBD in the central region where the velocity gradients are the largest. This has a strong impact on the quality of the fit but also on the final $M_{\mathrm{BH}}$ estimate. When the observed line surface 

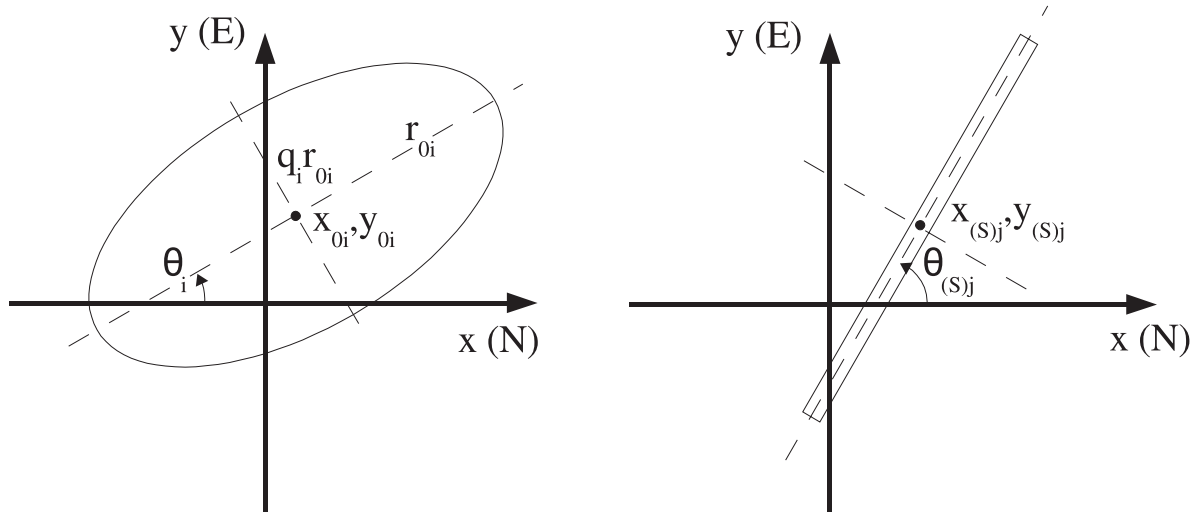

Fig. 14. Reference frame $x y$ in the plane of the sky. $x$ and $y$ are aligned along the North and East directions, respectively. Left: $x y$ characterization of the location of an elliptical isophote of the $i_{\mathrm{th}}$ component of the ISBD. Right: $x y$ characterization of the location of the $j_{\mathrm{th}}$ slit.

brightness distribution along the slit is strongly peaked in the nuclear region and has a profile which is little different from that of an unresolved source, as in the present case, one cannot simply use a deconvolved emission line image but should try to reproduce the observed line surface brightness distribution with a parameterized intrinsic one and taking into account the intrumental effects. Therefore we decided to parameterize the ISBD with a combination of analytic functions. We will show in the following analysis that the final result, i.e. the BH mass, is very little dependent on the assumed ISBD.

We adopt the reference system $x y$ in the plane of the sky where the $x$ and $y$ axes are aligned with the North and East directions, respectively (see Fig. 14). The total emission line surface brightness at a point $x, y$ is represented in our model by

$I(x, y)=\sum_{i} I_{0 i} f_{i}\left(\frac{r_{i}}{r_{0 i}}\right)$

where $I_{0 i}$ is the amplitude of the $i_{\text {th }}$ component which is described by the analytic function $f_{i}\left(r_{i} / r_{0 i}\right) . r_{0 i}$ is a scale radius and $r_{i}$ is the distance from a simmetry center defined by

$$
\begin{aligned}
x_{i}^{\prime} & =x-x_{0 i} \\
y_{i}^{\prime} & =y-y_{0 i} \\
x_{i}^{\prime \prime} & =x_{i}^{\prime} \cos \theta_{i}+y_{i}^{\prime} \sin \theta_{i} \\
y_{i}^{\prime \prime} & =\left(-x_{i}^{\prime} \cos \theta_{i}+y_{i}^{\prime} \sin \theta_{i}\right) / q_{i} \\
r_{i} & =\left[\left(x_{i}^{\prime \prime}\right)^{2}+\left(y_{i}^{\prime \prime}\right)^{2}\right]^{1 / 2} .
\end{aligned}
$$

Thus, the isophotes of the $i_{\text {th }}$ surface brightness component are ellipses centered in $\left(x_{0 i}, y_{0 i}\right)$, with axial ratio $q_{i}$ (minor over major axis) and major axis aligned along a direction with position angle PA $=\theta_{i}$ (Fig. 14). The adopted analytic functions are exponentials, Gaussians and constants defined as:

$$
\begin{aligned}
& f_{i}\left(r_{i}\right)=\exp \left(-r_{i} / r_{0 i}\right) \\
& f_{i}\left(r_{i}\right)=\exp \left[-\frac{1}{2}\left(r_{i} / r_{0 i}\right)^{2}\right] \\
& f_{i}\left(r_{i}\right)=1\left(r_{i}<=r_{01}\right) ; 0\left(r_{i}>r_{01}\right) .
\end{aligned}
$$

The ISBD of Eq. (6) is then convolved with the PSF of the system and integrated over apertures defined by the slit and the detector pixel size. The free parameters of the fit are determined with a $\chi^{2}$ minimization by comparing observed and model data. The position of the $j_{\text {th }}$ slit in the $x y$ reference system is characterized by $x_{(S) j}, y_{(S) j}$, the slit center position and $\theta_{(S) j}$ its position angle on the plane of the sky. We now derive the ISBDs for STIS and ISAAC data.

\subsubsection{STIS data}

The acquisition procedure followed during HST observations (i.e. centering on a bright star and slew to the expected nucleus location) ensures that the STIS slits are centered on the same point within an accuracy of a fraction of a pixel. Inspection of Fig. 4 then suggests that the ISBD should be described at least by 4 components: one at the slit center (the nuclear component), one offset by -0. '2 along the slit at VIS1, one at $-0 .{ }^{\prime} 6$ along the slit at VIS2 and a constant base. The flattening and orientation of the offnuclear components can be left as free parameters of the fit. However, in the case of a nuclear component with a size smaller than the spatial resolution it is safer to reduce the number of free parameters and therefore we have considered the following cases for the nuclear component:

(1) nuclear component circularly symmetric;

(2) nuclear component with PA $=32.5 \mathrm{deg}$ and $q=0.5$;

(3) nuclear component with $\mathrm{PA}=122.5 \mathrm{deg}$ and $q=0.5$;

(4) 2 nuclear components with PA $=77.5,-12.5 \mathrm{deg}$ and $q=0.5$

(5) 2 nuclear components with PA $=77.5,-12.5 \mathrm{deg}$ and $q=0.17$

(6) 2 nuclear components circularly symmetric;

(7) 2 nuclear components, one circularly symmetric and the other with $\mathrm{PA}=32.5 \mathrm{deg}$ and $q=0.5$;

(8) 2 nuclear components, one circularly symmetric and the other with $\mathrm{PA}=122.5 \mathrm{deg}$ and $q=0.5$;

(9) 2 nuclear components with PA $=32.5,122.5 \mathrm{deg}$ and $q=0.5$.

$\mathrm{PA}=32.5 \mathrm{deg}$ is the PA of the Centaurus A jet and is the direction along which $\mathrm{Pa} \alpha$ and [Fe II] emissions are elongated and $q=0.5$ is their axial ratio (Schreier et al. 1998; Marconi et al. 2000). PA $=122.5 \mathrm{deg}$ is the direction perpendicular to the previous one. In the case of 2 components with $\mathrm{PA}=77.5,-12.5 \mathrm{deg}$ we mimick an ionization cone with axis at $\mathrm{PA}=32.5 \mathrm{deg}$ and opening angle of $45 \mathrm{deg}$. 


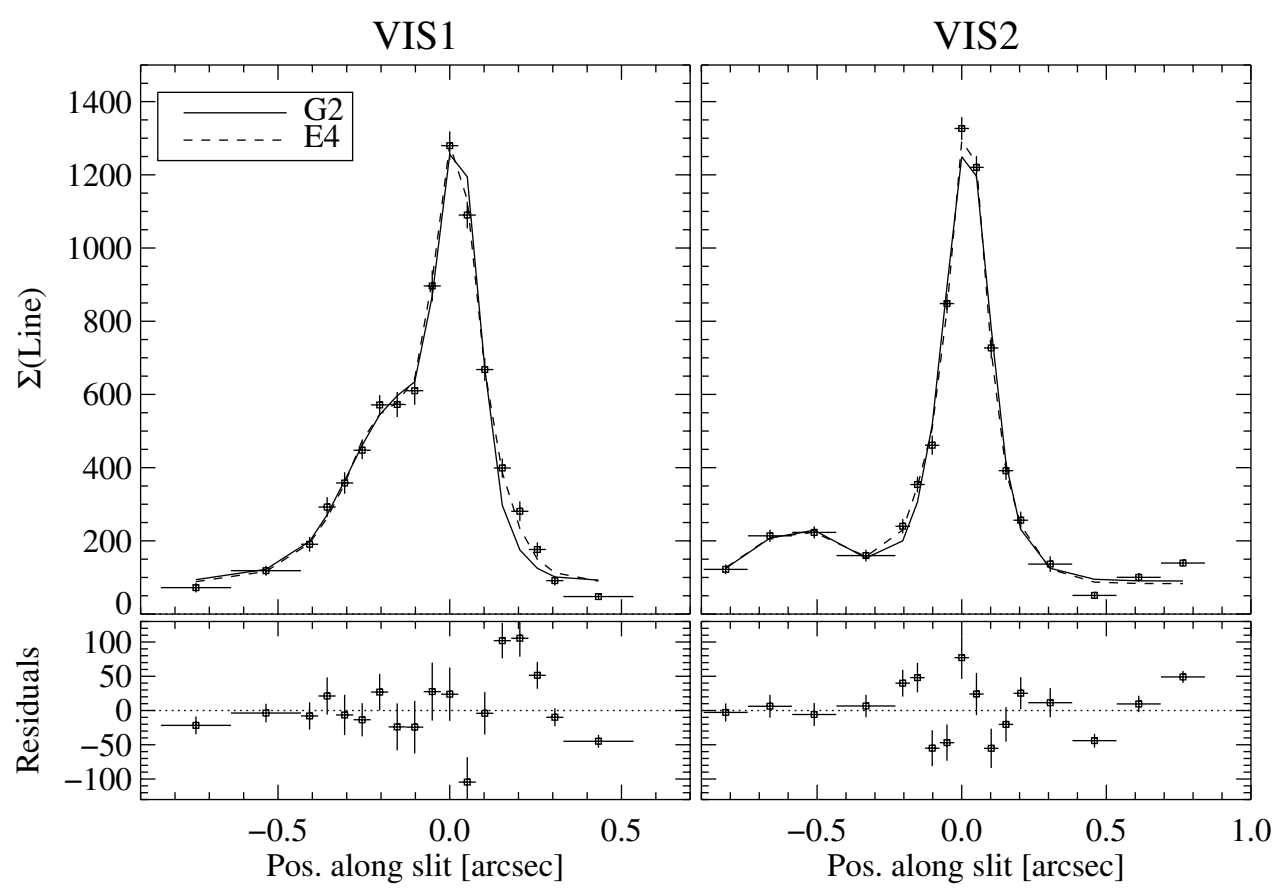

Fig. 15. Fit of the STIS line surface brightness distribution along the slit for 2 sample cases.

Table 2. Intrinsic line surface brightness distributions for STIS.

\begin{tabular}{llrrrrrrr}
\hline \hline id & function & $i$ & $I_{0 i}$ & $r_{0 i}$ & $x_{0 i}$ & $y_{0 i}$ & $\theta_{i}$ & $q_{i}$ \\
\hline G2 & Gauss & 1 & 3685 & 0.145 & 0.028 & 0.004 & $32.5^{\star}$ & $0.5^{\star}$ \\
& Gauss & 2 & 1950 & 0.231 & -0.178 & 0.196 & $0.00^{\star}$ & $1.0^{\star}$ \\
& Gauss & 3 & 295 & 0.278 & -0.481 & -0.311 & $0.00^{\star}$ & $1.0^{\star}$ \\
& const & 4 & 90 & & & & & \\
\hline E4 & expo & 1 & 2438 & 0.066 & 0.016 & 0.036 & $77.5^{\star}$ & $0.5^{\star}$ \\
& expo & 2 & 3333 & 0.056 & 0.023 & 0.006 & $-12.5^{\star}$ & $0.5^{\star}$ \\
& expo & 3 & 1948 & 0.058 & -0.217 & 0.103 & $0.00^{\star}$ & $1.0^{\star}$ \\
& expo & 4 & 440 & 0.097 & -0.458 & -0.352 & $0.00^{\star}$ & $1.0^{\star}$ \\
& const & 5 & 83 & & & & & \\
\hline
\end{tabular}

^ Fixed parameter.

For each of the above cases we have used exponential (E), Gaussian $(G)$ and constant (C) functions. An ISBD is then labeled as, e.g., E5 which means exponential functions in case 5 above.

Sample results of the fitting procedure are shown in Fig. 15 for the ISBDs G2 and E4 and the best fit parameters for these ISBDs are shown in Table 2. Inspection of Fig. 15 indicates that ISBDs G2 and E4, though different, result in very similar observed surface brightness distributions.

\subsubsection{ISAAC data}

The observational procedure followed during ISAAC observations (i.e. centering the slit on the $K$ band peak, Marconi et al. 2001) ensures that the three slits are centered on the same point within a fraction of the ISAAC pixel. However it is not clear if the emission line peak observed in $\mathrm{Pa} \beta$ and [Fe II] is coincident with the continuum peak. Schreier et al. (1998) analyze a $\mathrm{Pa} \alpha$ image and find that the continuum subtracted line image has a point like emission (at NICMOS resolution) which is concident with that of the continuum. The same happens for [Fe II] at $1.6 \mu \mathrm{m}$ (Marconi et al. 2000). Regardless of these indications in order to avoid biases in the final results, we consider two cases, when the slits are centered on the emission line peak (coincident with the continuum peak) and when they are not.

The seeing of the observations can be estimated from the unresolved continuum source observed along the slit in the ISAAC observations. However, the unresolved continuum source in the $J$ band is not as strong as in the $K$ band and the estimate of the seeing can be uncertain by as much as 0 . $^{\prime} 1$. We parameterize the seeing as a Gaussian with given $F W H M$ (Full Width at Half Maximum) and we consider 3 cases for the FWHM values:

- seeing 0.' 4 for PA1, PA2 and seeing 0.'5 for PA3;

- seeing 0.'45 for PA1, PA2 and seeing 0.'55 for PA3;

- seeing 0.' 5 for PA1, PA2 and PA3.

Inspection of Fig. 6 suggests that the ISBDs of $\mathrm{Pa} \beta$ and [Fe II] can be described with 4 components: a nuclear component, a disk component (the one associated with the extended nuclear feature observed in Pa $\alpha$ by Schreier et al. 1998), and an extended one. The flattening and orientation of the disk components are fixed to $q=0.5$ and $\theta=32.5$, i.e. the values characterizing the $\operatorname{Pa} \alpha$ feature, and the extended component is assumed circularly symmetric. We then parameterize the nuclear component according to the following 4 cases:

1. nuclear component circularly symmetric;

2. nuclear component with $\mathrm{PA}=32.5 \mathrm{deg}$ and $q=0.5$;

3. nuclear component with $\mathrm{PA}=122.5 \mathrm{deg}$ and $q=0.5$;

4. 2 nuclear components with $\mathrm{PA}=77.5,-12.5 \mathrm{deg}$ and $q=0.5$.

The adopted values of PA and $q$ were chosen as described in the previous section. For each of the above cases we have used 


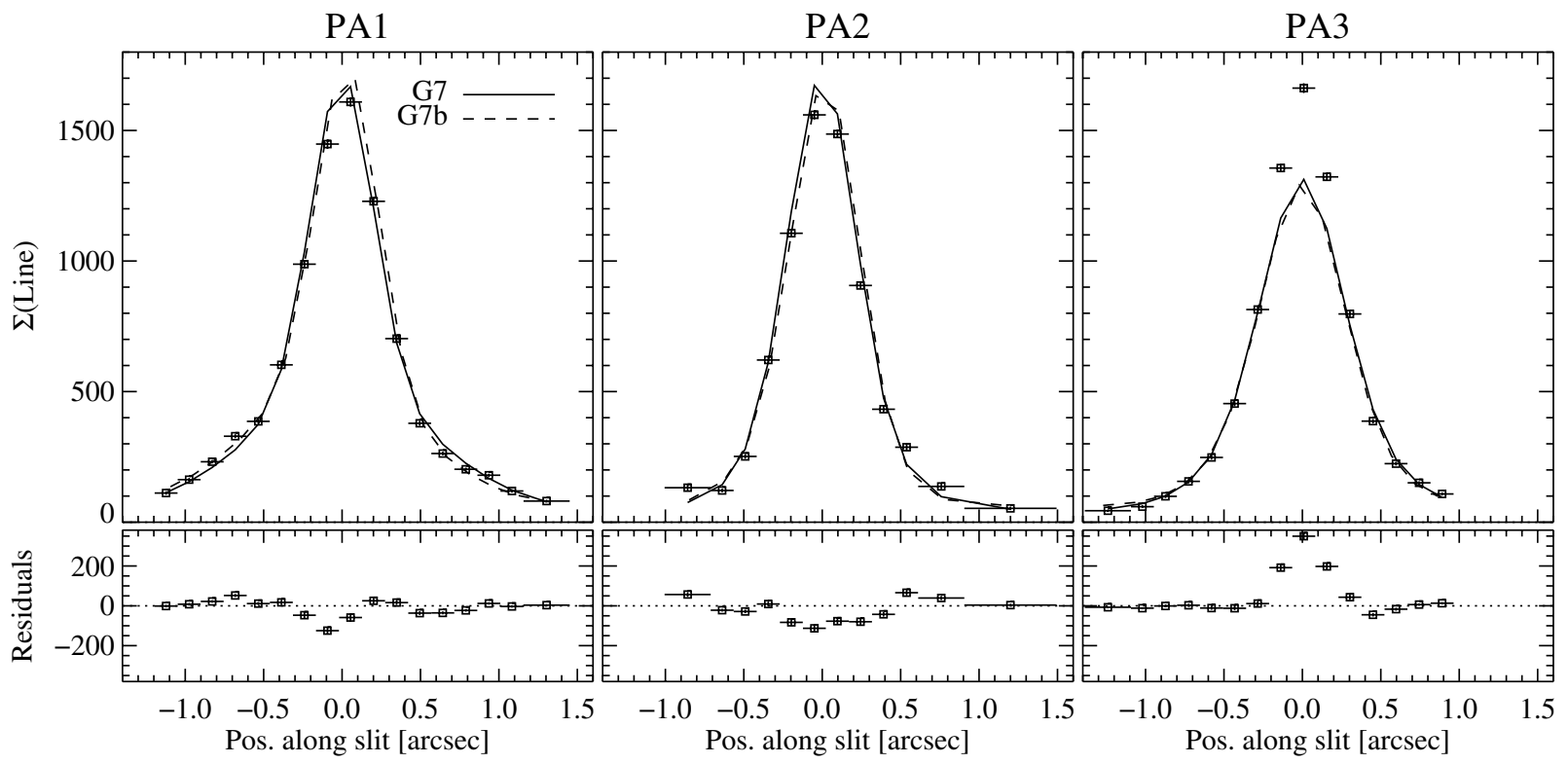

Fig. 16. Fit of the ISAAC line surface brightness distribution along the slit for 2 sample cases.

Table 3. Intrinsic line surface brightness distributions for ISAAC.

\begin{tabular}{llrrrrrrr}
\hline \hline id & Function & $i$ & $I_{0 i}$ & $r_{0 i}$ & $x_{0 i}$ & $y_{0 i}$ & $\theta_{i}$ & $q_{i}$ \\
\hline G7 & Gauss & 1 & 40500 & 0.119 & $0.00^{\star}$ & $0.00^{\star}$ & $122.5^{\star}$ & $0.5^{\star}$ \\
& Gauss & 1 & 591 & 1.262 & $0.00^{\star}$ & $0.00^{\star}$ & $32.5^{\star}$ & $0.5^{\star}$ \\
& Gauss & 1 & 68 & 3.407 & $0.00^{\star}$ & $0.00^{\star}$ & $0.00^{\star}$ & $1.0^{\star}$ \\
\hline G7b & Gauss & 1 & 41184 & 0.121 & 0.106 & 0.009 & $12.5^{\star}$ & $0.5^{\star}$ \\
& Gauss & 1 & 627 & 1.190 & $0.00^{\star}$ & $0.00^{\star}$ & $32.5^{\star}$ & $0.5^{\star}$ \\
& Gauss & 1 & 64 & 9.478 & $0.00^{\star}$ & $0.00^{\star}$ & $0.00^{\star}$ & $1.0^{\star}$ \\
\hline
\end{tabular}

^ Fixed parameter.

exponential (E), Gaussian (G) and constant (C) functions. A surface brightness distribution is labeled by assigning a letter $\mathrm{E}, \mathrm{G}$ or $\mathrm{C}$ according to the functional form used, a number 1-4 (seeing case 1), 5-8 (seeing case 2) or 9, a-c (seeing case 3 ). The label is further characterized by " $b$ " if we are in the case when the emission line peak is not coincident with the slit centers. Therefore E5 indicates, for instance, exponential functions with surface brightness case 1 and seeing case 5 while $\mathrm{G} 2 \mathrm{~b}$ indicates Gaussian functions in surface brightness case 2 and seeing case 1 .

Sample results of the fitting procedure are shown in Fig. 16 for ISBD G7 and E7 and the best fit parameters for these ISBDs shown in Table 3.

In Fig. 17 we plot the isophotal contours of the ISBDs used in Figs. 15 and 16. These ISBDs have been chosen as representative because they provide the best fits of STIS velocities (Table 4) and of ISAAC velocities and velocity dispersions (Table 6). From the figure the centrally peaked components are clearly distinguished from the more extended ones. The central strong components of the ISBDs adopted for the STIS data is elongated along the jet axis, while the ISAAC ones are perpendicular to it. The more extended component in the ISAAC data represents the disk like feature seen in $\operatorname{Pa} \alpha$ by Schreier et al. (1996) while it seems that this component is missing in
[S III] which shows a different morphology from those of $\mathrm{Pa} \beta$ and [Fe II].

\subsection{Kinematical fitting of the rotation curves}

In principle, in order to constrain the $\mathrm{BH}$ mass, one should compare the emission line profile predicted by the model with the observed one. However, as discussed at length by Marconi et al. (2003a), our approach is to compare the moments of the line profiles: the average velocity $\langle v\rangle$ and velocity dispersion $\sigma$ defined as $\sigma^{2}=\left\langle v^{2}\right\rangle-\langle v\rangle^{2}$.

This approach requires that the parameterized line profile, adopted in the fitting process, is able to reproduce the shape of the observed line profiles within the noise (see also Barth et al. 2001). This condition is satisfied for STIS data were the observed line profile is well represented by a single Gaussian, after deblending the "blue" component which is obviously not emitted by circularly rotating gas. The above condition is also satisfied for the ISAAC data where the observed line profiles in the nuclear region are well matched with the parameterization described above. The Hermite expansion has the obvious advantage of allowing an excellent description of the line profiles without introducing the instability and the larger number of parameters (6 instead of 5) typical of fits with two Gaussian function. To our knowledge this is the first time that the Hermite expansion is applied to the analysis of emission lines.

In order to be compared with the observations, model $\langle v\rangle$ and $\sigma$ are computed taking into account the spatial resolution of the observations and the size of the apertures which are used to measure the observed quantities. The formulae used to compute velocities, widths and line surface brightness distributions and the details of their derivation, numerical computation and model fitting are described in details in Appendix B of Marconi et al. (2003a). 

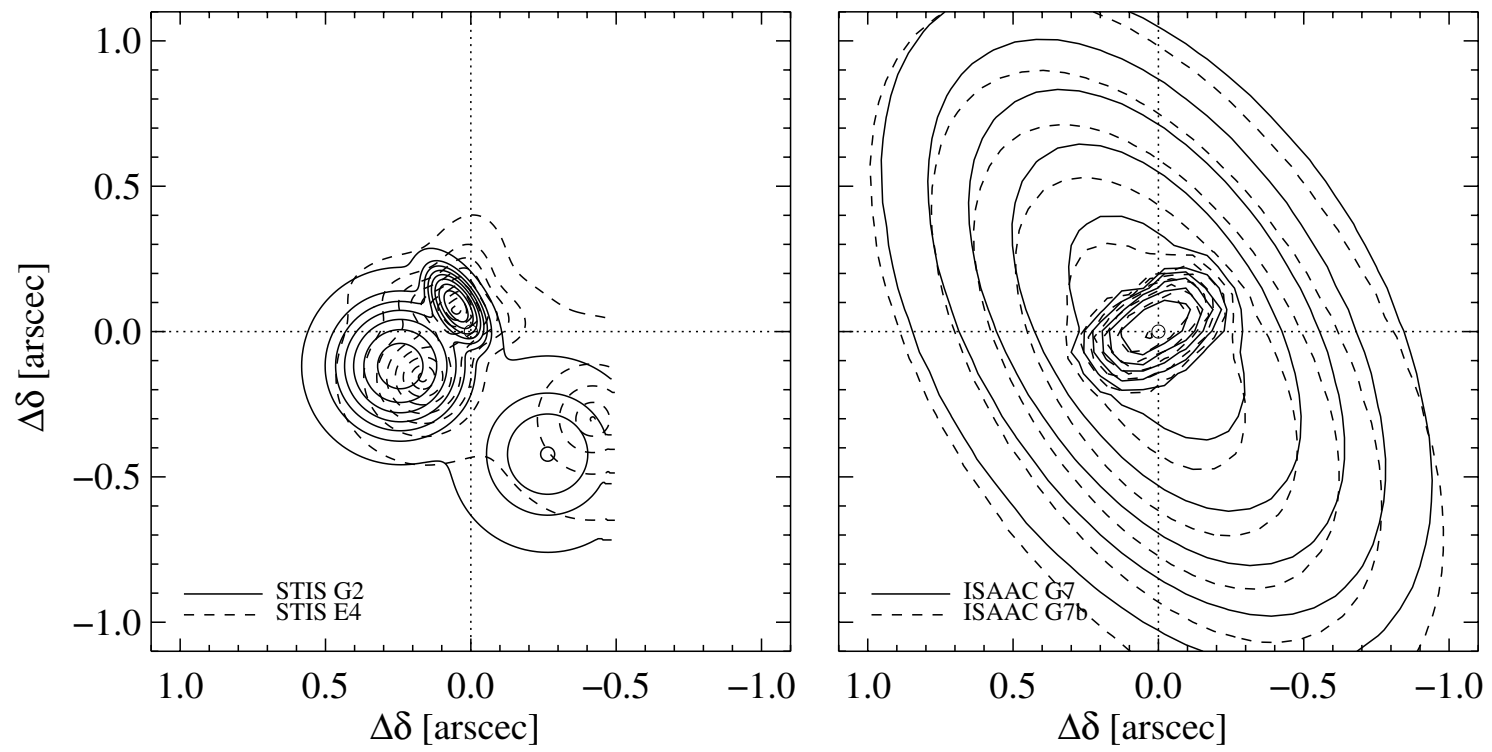

Fig. 17. Left: isophotal contour levels of the intrinsinc surface brightness distributions G2 and E4 used in Fig. 15 to reproduce the STIS data. The ISBDs have not been convolved with the instrumental response. Contour levels start from -2 and go to -0.2 in log of the peak of the ISBD. The filled circle indicate the location of the slit centers. Right: as in the left panel but for the ISBDs G7 and G7b used in Fig. 16 to reproduce the ISAAC data.

Table 4. Fit results from the analysis of STIS data with $i=25 \mathrm{deg}$.

\begin{tabular}{|c|c|c|c|c|c|c|c|c|}
\hline Flux $^{a}$ & $x_{0}$ & $y_{0}$ & $\log M_{\mathrm{BH}}$ & $\log M / L$ & $\theta$ & $v_{\text {sys }}$ & $\chi_{\mathrm{red}}^{2}$ & $\left(\chi_{\text {red }}^{2}\right)_{\text {resc }}^{b}$ \\
\hline \multicolumn{9}{|c|}{ Fit of velocity $\left(\Delta v_{0}=10.4 \mathrm{~km} \mathrm{~s}^{-1}\right)^{c}$} \\
\hline G2 & -0.05 & -0.05 & 8.00 & $0.00^{\star}$ & 161.0 & 554.9 & 1.27 & 1.00 \\
\hline E4 & -0.06 & -0.04 & 7.89 & $0.00^{\star}$ & 167.0 & 547.3 & 1.30 & 1.05 \\
\hline G1 & -0.06 & -0.04 & 7.93 & $0.00^{\star}$ & 162.6 & 548.7 & 1.32 & 1.05 \\
\hline E6 & -0.06 & -0.03 & 7.89 & $0.00^{\star}$ & 163.6 & 547.1 & 1.35 & 1.08 \\
\hline G6 & -0.06 & -0.03 & 7.89 & $0.00^{\star}$ & 164.3 & 545.5 & 1.37 & 1.09 \\
\hline E7 & -0.06 & -0.03 & 7.88 & $0.00^{\star}$ & 163.9 & 546.1 & 1.41 & 1.13 \\
\hline E8 & -0.05 & -0.04 & 7.87 & $0.00^{\star}$ & 168.7 & 543.4 & 1.41 & 1.14 \\
\hline E9 & -0.05 & -0.03 & 7.86 & $0.00^{\star}$ & 169.5 & 544.1 & 1.42 & 1.15 \\
\hline G7 & -0.06 & -0.03 & 7.90 & $0.00^{\star}$ & 162.9 & 545.1 & 1.48 & 1.17 \\
\hline E2 & -0.06 & -0.04 & 7.95 & $0.00^{\star}$ & 161.3 & 548.5 & 1.51 & 1.18 \\
\hline C6 & -0.06 & -0.03 & 7.93 & $0.00^{\star}$ & 161.1 & 546.2 & 1.51 & 1.20 \\
\hline E1 & -0.06 & -0.04 & 7.91 & $0.00^{\star}$ & 169.1 & 543.4 & 1.50 & 1.20 \\
\hline E5 & -0.04 & -0.05 & 7.91 & $0.00^{\star}$ & 178.9 & 541.2 & 1.49 & 1.21 \\
\hline $\mathrm{C} 1$ & -0.05 & -0.02 & 7.80 & $0.00^{\star}$ & 168.9 & 539.7 & 1.55 & 1.24 \\
\hline E3 & -0.05 & -0.03 & 7.84 & 0.00 * & 173.8 & 538.7 & 1.60 & 1.29 \\
\hline G4 & -0.04 & -0.04 & 7.89 & $0.00^{\star}$ & 177.5 & 539.0 & 1.60 & 1.30 \\
\hline G8 & -0.05 & -0.04 & 7.86 & 0.00 * & 172.9 & 539.2 & 1.65 & 1.33 \\
\hline $\mathrm{C} 8$ & -0.05 & -0.03 & 7.81 & $0.00^{\star}$ & 170.5 & 540.1 & 1.69 & 1.36 \\
\hline $\mathrm{C} 4$ & -0.05 & -0.04 & 7.85 & $0.00^{\star}$ & 174.8 & 544.4 & 1.72 & 1.40 \\
\hline G3 & -0.05 & -0.02 & 7.82 & $0.00^{\star}$ & 174.2 & 537.1 & 1.77 & 1.43 \\
\hline G9 & -0.05 & -0.05 & 7.93 & $0.00^{\star}$ & 174.3 & 537.1 & 1.80 & 1.44 \\
\hline C7 & -0.05 & -0.01 & 7.79 & $0.00^{\star}$ & 160.4 & 545.4 & 1.86 & 1.47 \\
\hline Average $^{d}$ & $-0.05 \pm 0.01$ & $-0.03 \pm 0.01$ & $7.88 \pm 0.05$ & & $168.4 \pm 5.7$ & $543.7 \pm 4.3$ & & \\
\hline
\end{tabular}

a Adopted ISBD.

${ }^{b}$ Rescaled $\chi^{2}$ with errors computed as $\Delta v_{i}^{\prime 2}=\Delta v_{i}^{2}+\Delta v_{0}{ }^{2}$.

${ }^{c}$ Systematic error adopted to renormalize $\chi^{2}$.

${ }^{d}$ Average and rms of best fit parameter values.

* Parameter was held fixed. 
In Appendix A we describe a problem in the computation of kinematical quantities which might derive from subsampling. We also present a way to overcome this problem and therefore our computations are not affected by it.

Thus, model $\langle v\rangle$ and $\sigma$ depend on $\Sigma$, the ISBD of the emission lines defined in the previous section, and on the following parameters:

- $x_{0}, y_{0}$, the position of the kinematical center in the plane of the sky with the reference frame defined in the previous section;

- $i$, the inclination of the rotating disk;

$-\theta$, the position angle of the disk line of nodes;

- $v_{\text {sys }}$, the systemic velocity of the disk;

- $M / L$, the mass-to-light ratio of the stellar population;

- $M_{\mathrm{BH}}$, the BH mass.

Not all of these parameters can be independently determined by fitting the observations. It can be inferred from the equations in Appendix B of Marconi et al. (2003a) that $M_{\mathrm{BH}}, M / L$ and $i$ are directly coupled and this has been discussed in detail by Macchetto et al. (1997) and Marconi et al. (2003a). To avoid problems deriving from this coupling we work here at fixed disk inclination $i$.

\subsubsection{STIS data}

We fit the STIS rotation curves using all the ISBDs determined in Sect. 4.2.1 and, at first, we work with fixed inclination $i=25 \mathrm{deg}$. The STIS PSF used in the computations is obtained at $\lambda 9550 \AA$ with TinyTIM (Krist \& Hook 1999). The free parameters of the fit $\left(x_{0}, y_{0}, \log M_{\mathrm{BH}}, \theta, v_{\mathrm{sys}}\right)$ are obtained with a $\chi^{2}$ minimization. Since, as shown below, the contribution of the stellar mass to the gravitational potential is negligible we worked with fixed $M / L=1$ in order to reduce the number of free parameters. We will show that this assumption is consistent with the data, when searching for the confidence limits on the parameters. During the fitting process, velocity corrections are applied to the VIS1 and VIS2 data in order to take into account errors on absolute wavelegth calibration. The best fit parameters for all models with acceptable $\chi^{2}$ are shown in Table 4 and in Fig. 18 (top panels) where we compare the observations and the 2 models with the lowest $\chi^{2}$. In order to select the "good" models shown in Table 4 we first renormalize the $\chi^{2}$ following a procedure already adopted by Barth et al. (2001) and Marconi et al. (2003a). The fit with the lowest $\chi^{2}$ is provided by the model with ISBD "G2" which has $\chi_{\text {red }}^{2}=1.27(27.9 / 22)$. This $\chi_{\text {red }}^{2}$ value is already acceptable at the $95 \%$ confidence level given the number of degrees of freedom ( 22 d.o.f.) but we conservatively assume that there is an extra error in velocity, $\Delta v_{0}$, which has not been taken into account and that must be added in quadrature to the data. $\Delta v_{0}$ might also represent an intrinsic spread in velocity due, for instance, to non-circular local motions superimposed to the large scale rotation. $\Delta v_{0}$ is chosen such the best fit model has $\chi_{\text {red }}^{2}=1$. For model "G2", $\Delta v_{0}=10.4 \mathrm{~km} \mathrm{~s}^{-1}$ and this is a small value compared to the errors on STIS velocities indicating the goodness of the fit. We then renormalize $\chi^{2}$ for all models using the above value of $\Delta v_{0}$ and we consider acceptable only the models with a $\leq 95 \%$ significance level, i.e. those with $\chi_{\text {red }}^{2} \leq 1.55$ for 22 d.o.f. For these models, the velocity shifts applied to the data are on average $-2.5 \mathrm{~km} \mathrm{~s}^{-1}( \pm 2.6 \mathrm{rms})$ and $2.6 \mathrm{~km} \mathrm{~s}^{-1}( \pm 2.7 \mathrm{rms})$ for VIS1 and VIS2 respectively. These are lower than the expected errors on the absolute wavelength calibration (e.g. Kim Quijano et al. 2003). The models shown in Fig. 18 are in good agreement with the observations as confirmed by the low $\chi_{\text {red }}^{2}$ found prior to rescaling. In particular the models nicely reproduce the steep velocity gradients and turnoffs observed in the rotation curves which are the signature of the $\mathrm{BH}$. The velocity fall-off in VIS1 is slightly faster than keplerian but this disagreement appears in points with low signal-to-noise ratio. The best fit model is characterized by a $\mathrm{BH}$ mass of $\log \left(M_{\mathrm{BH}} / M_{\odot}\right)=8$ and by a disk line of nodes with $\mathrm{PA}=161 \mathrm{deg}$. The dotted line in the figure shows the stellar contribution to the rotation curve, i.e. what would be observed without a BH. Clearly, the high velocity gradients can be explained only with the presence of a BH. The location of the kinematical center is $-00^{\prime} 05,-0{ }^{\prime} 05$ with respect to the location of the slit centers.

In order to estimate statistical errors associated with the best fit parameters, we present in Figs. 19a,b $\chi^{2}$ contours for the joint variation of $M_{\mathrm{BH}}-M / L$ and of $M_{\mathrm{BH}}-\theta$. The $\chi^{2}$ grids have been computed with ISBD "G2", fixing 2 interesting parameters $\left(M_{\mathrm{BH}}, M / L\right.$ or $\left.M_{\mathrm{BH}}, \theta\right)$ and varying the others to minimize $\chi^{2}$. Before computing confidence levels we have renormalized $\chi^{2}$ as described above so that the model with the lowest $\chi^{2}$ value has $\chi_{\text {red }}^{2}=1$. Confidence levels for 2 interesting parameters are then found following Avni (1976): $\chi^{2}=\chi_{\min }^{2}+2.3$, $4.61,6.17$ for confidence levels $68.3 \%, 90 \%$ and $95.4 \%$, respectively. Statistical errors are \pm 0.1 on $\log M_{\mathrm{BH}}$ and \pm 6 on $\theta$. Figure 19a shows that it is not possible to determine $M / L$ but only place an upper limit on it $(M / L<1.6)$. Also, the same figure justifies our choice of fixing $M / L=1$, which is within the $1 \sigma$ confidence contour.

By comparing the results of the model fitting performed with different ISBDs we can have an estimate of the systematic errors associated with the choice of the ISBD. In the last row of Table 4 we show the average values of the fit parameters and the associated rms. It is highly significant that the systematic errors on all parameters and in particular on BH mass are very small when considering all the adopted ISB. The average $\mathrm{BH}$ mass is $\log M_{\mathrm{BH}}=7.88$ with rms of 0.05 .

In conclusion, for $i=25 \mathrm{deg}$, the BH mass estimate from STIS data with ISBD “G2" is $\log M_{\mathrm{BH}}=8.0 \pm 0.1( \pm 0.05$ systematic) and $\theta=161 \pm 6$ ( \pm 6 systematic).

In Fig. 19c and Table 5 we adopt ISBD G2 and we explore how the minimum $\chi^{2}$ depends on disk inclination. As above, we have renormalized so that the model with the minimum $\chi^{2}$ value has $\chi_{\text {red }}^{2}=1$. The STIS data favour inclinations $i<48 \mathrm{deg}$ at a $95 \%$ confidence level, a result already found by Marconi et al. (2001).

In Fig. $18 \mathrm{~b}$ we plot the expected velocity dispersion for the 2 best fit models plotted in Fig. 18a. A constant intrinsic velocity dispersion of $\sigma_{0}=160 \mathrm{~km} \mathrm{~s}^{-1}$ was added to match the "plateau" observed at VIS1 (at $9500 \AA$ the degradation of the spectral resolution introduced by the grating and slit width is $\sim 160 \mathrm{~km} \mathrm{~s}^{-1}$, see Sect. 2.1). In both cases an increase in $\sigma$ is observed at the nucleus and this is roughly matched with 

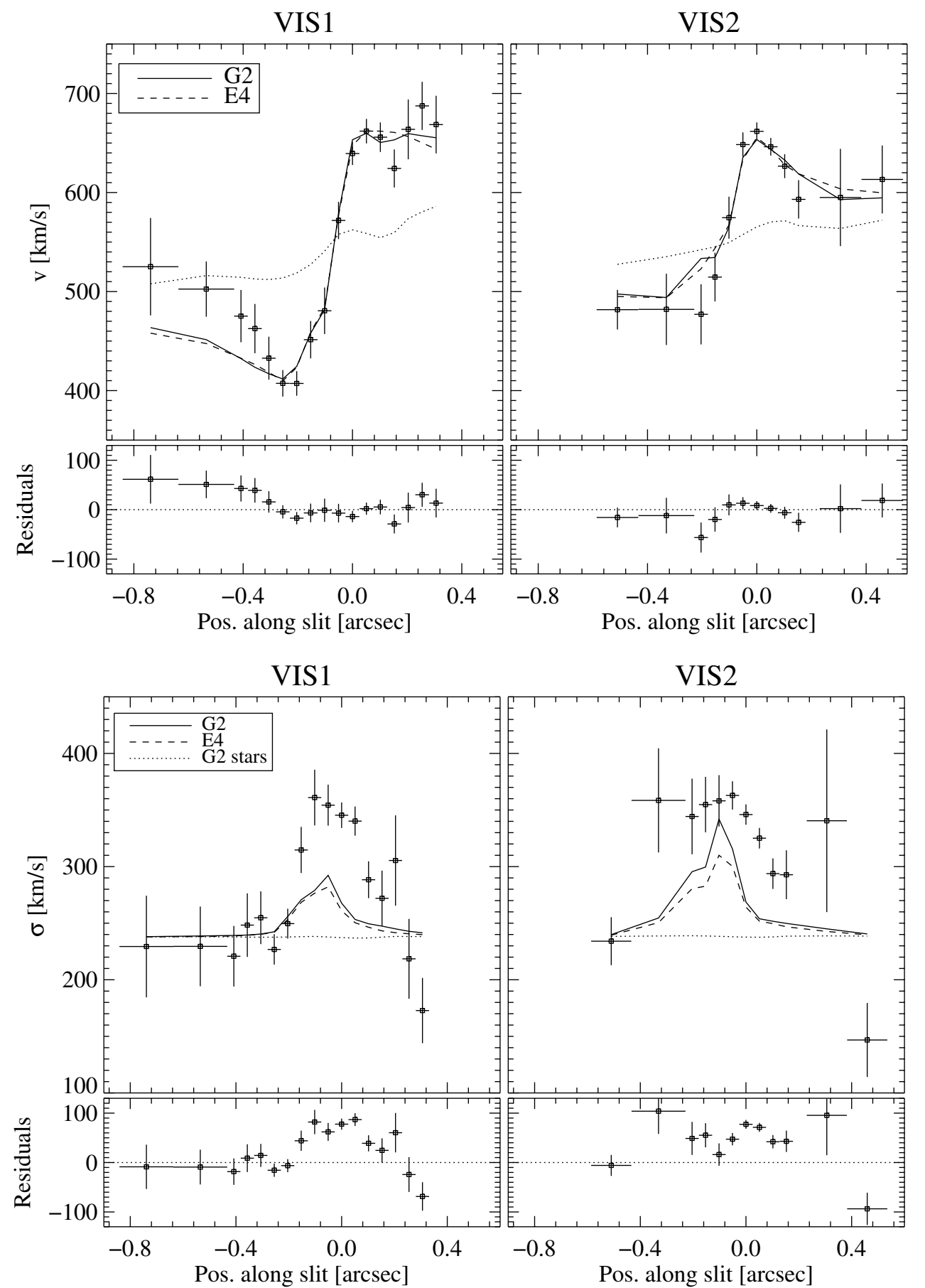

Fig. 18. Top panels: fit of the STIS velocities along the slit. The empty squares with error bars represent the observed values while the solid lines connect corresponding model values. Dotted lines represent the contribution of the mass in stars to the rotation curve, i.e. what would be observed without a BH. "G2" and "E4" are the two ISBDs which provide the best fit models with the lowest $\chi^{2}$. Bottom panels: velocity dispersions expected from the two best fit models compared with the observed ones. Notation as in the upper panels.

unresolved rotation only at VIS2. At VIS1 unresolved rotation explains only $\sim 80 \%$ of observed $\sigma$ nuclear increase but the adopted $\sigma_{0}=160 \mathrm{~km} \mathrm{~s}^{-1}$ is smaller than the contribution from unresolved rotation.

\subsubsection{ISAAC data}

We fit the ISAAC rotation curves using all ISBDs determined in Sect. 4.2.2. We proceed as in the previous section and we work at fixed inclination, $i=25 \mathrm{deg}$. The PSF used in the computations is a Gaussian with $F W H M$ equal to the adopted seeing. The free parameters of the fit $\left(x_{0}, y_{0}\right.$, $\left.\log M_{\mathrm{BH}}, \log M / L, \theta, v_{\text {sys }}\right)$ are obtained with a $\chi^{2}$ minimization. During the fitting process, velocity corrections are applied to the PA1, PA2 and PA3 data in order to take into account errors on absolute wavelegth calibration. The best fit parameters for all models with acceptable $\chi^{2}$ are shown in the first part of Table 6 ("Fit of velocity") and in Fig. 20 

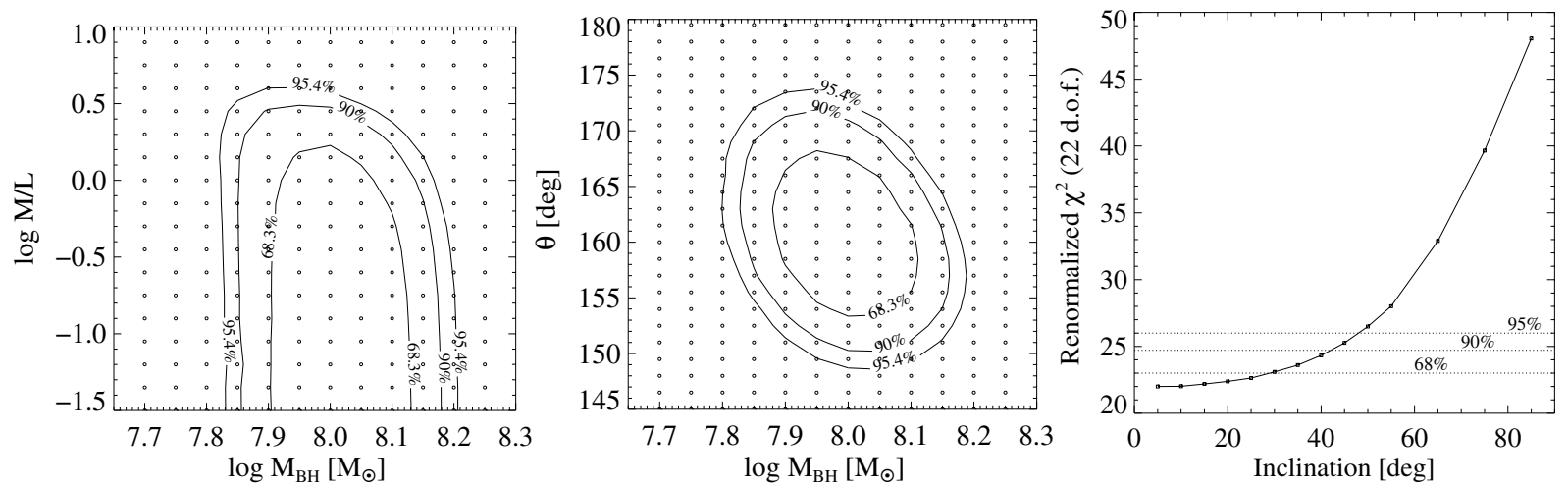

Fig. 19. a) $\chi^{2}$ contours for the joint variation of $M_{\mathrm{BH}}$ and $M / L$ using the STIS data (ISBD G2). Contour levels are for $\chi^{2}=\chi_{\min }^{2}+2.3,4.61$, 6.17 corresponding to $68.3 \%, 90 \%$ and $95.4 \%$ confidence levels. The dot indicates the $M_{\mathrm{BH}}$ and $M / L$ values for which the $\chi^{2}$ minimization was computed. b) same as a) but for $M_{\mathrm{BH}}-\theta$. c) Dependence of $\chi^{2}$ on the adopted disk inclination. Dotted lines indicate the $68.3 \%, 90 \%$ and $95.4 \%$ confidence levels.

Table 5. Effect of $i$ variation on best fit parameters and minimum $\chi^{2}$ for STIS data. All models adopt ISBD G2. Confidence levels $68.3 \%$, $90 \%$ and $95.4 \%$ are at $\chi^{2}=\chi_{\min }^{2}+1.0,2.71,4.0$, for one interesting parameter (Avni 1976).

\begin{tabular}{lcccc}
\hline \hline$i$ & $\log M_{\mathrm{BH}}$ & $\log \left(M_{\mathrm{BH}} \sin ^{2} i\right)$ & $\theta$ & $\chi_{\text {resc }}^{2}$ \\
\hline 5. & 9.35 & 7.23 & 159.1 & 22.0 \\
10. & 8.75 & 7.23 & 159.6 & 22.0 \\
15. & 8.41 & 7.24 & 159.6 & 22.2 \\
20. & 8.18 & 7.25 & 159.7 & 22.4 \\
25. & 8.00 & 7.25 & 161.0 & 22.6 \\
30. & 7.87 & 7.27 & 160.8 & 23.1 \\
35. & 7.76 & 7.27 & 162.1 & 23.6 \\
40. & 7.68 & 7.29 & 162.6 & 24.3 \\
45. & 7.62 & 7.32 & 163.8 & 25.3 \\
50. & 7.56 & 7.33 & 163.7 & 26.5 \\
55. & 7.52 & 7.34 & 165.0 & 28.0 \\
65. & 7.55 & 7.46 & 164.7 & 32.9 \\
75. & 7.64 & 7.61 & 161.9 & 39.7 \\
85. & 8.17 & 8.17 & 160.9 & 48.0 \\
\hline
\end{tabular}

we compare the observations and the 2 models with the lowest $\chi^{2}$.

In order to select the "good" models shown in Table 6 we renormalize the $\chi^{2}$ following the approach described in the previous section. The fit with the lowest $\chi^{2}$ is provided by the model with ISBD "G1" which has $\chi_{\text {red }}^{2}=2.62(91.9 / 35)$ and must be then renormalized with $\Delta v_{0}=6.3 \mathrm{~km} \mathrm{~s}^{-1}$. We then consider as acceptable all the models with a significance of $95 \%$, i.e. those with $\chi_{\text {red }}^{2}<1.37$ (35 d.o.f.). For the models in Table 6 the average velocity shifts applied to the data are $-1.0 \pm 3.4$, $-18.7 \pm 4.4$ and $10.9 \pm 5.7$ for PA1, PA2 and PA3 respectively. These are in very good agreement with the expected errors on the absolute wavelength calibration (Marconi et al. 2001).

As for the STIS data, the models reproduce the observed velocities and, in particular, they match the steep velocity gradients and turnoffs observed in the rotation curves which are the signature of any $\mathrm{BH}$. The best fit model is characterized by a $\mathrm{BH}$ mass of $\log \left(M_{\mathrm{BH}} / M_{\odot}\right)=8.14$ and by a disk line of nodes with $\mathrm{PA}=164 \mathrm{deg}$. The location of the kinematical center is $-0.08,-0.16$ with respect to the location of the slit centers.

In the lower panel of Fig. 20 we plot the expected velocity dispersion for the 2 lowest $\chi^{2}$ models. A constant intrinsic velocity dispersion of $\sigma_{0}=70 \mathrm{~km} \mathrm{~s}^{-1}$ was added to match the "plateau" observed at PA1. In both cases an increase in $\sigma$ is observed at the nucleus but this is clearly not matched by the models with unresolved rotation.

In order to verify if the observed $\sigma$ increase can be reproduced by the models we also perfomed the fits by considering velocity and velocity dispersion at the same time. Best fits acceptable to the $95 \%$ level (i.e., with 75 d.o.f., renormalized $\chi_{\text {red }}^{2} \leq 1.28$ ) are shown in the second part of Table 6 ("Fit of velocity and velocity dispersion") and in Fig. 21 we compare the observations and the 2 models with the lowest $\chi^{2}$. Though the fit of velocity is not as good as before, the observed curves are still acceptably reproduced by models. However this time models are also able to match the observed sigma increase showing that it can be explained by unresolved rotation. The reason for this result is that in the previous case the center of rotation was pushed far from the surface brightness peak in order to better reproduce velocities. Now the center of rotation is almost coincident (within $\sim 0$ '01) with the line emission peak (note that in the case of ISBDs " $b$ " the emission line peak is offset from the slit centers by approximately $0.1,-0.02$ and this is where the kinematical center is).

In Fig. 22 we show the $\chi^{2}$ contours for joint variation of $M_{\mathrm{BH}}, M / L$ and $M_{\mathrm{BH}}, \theta$ in order to estimate the statistical errors. The models have been computed adopting ISBD G7, fixing 2 interesting parameters and varying the others. The $\chi^{2}$ has been renormalized as before.

The statistical errors associated to the $\mathrm{BH}$ mass determination are \pm 0.05 in $\log M_{\mathrm{BH}}$ comparable to the systematic errors due to the adopted ISBD. As for the fit of the STIS data Fig. 22 shows that it is not possible to determine $M / L$ but one can only place an upper limit of $M / L<1$. The statistical errors associated to $\theta$ are $\pm 3 \mathrm{deg}$.

By comparing the results of the model fitting performed with ISBDs we can have an estimate of the systematic errors associated with the choice of the ISBD. In Table 6 we also 
Table 6. Fit results from the analysis of ISAAC data with $i=25 \mathrm{deg}$.

\begin{tabular}{|c|c|c|c|c|c|c|c|c|}
\hline Flux $^{a}$ & $x_{0}$ & $y_{0}$ & $\log M_{\mathrm{BH}}$ & $\log M / L$ & $\theta$ & $v_{\text {sys }}$ & $\chi_{\text {red }}^{2}$ & $\left(\chi_{\text {red }}^{2}\right)_{\text {resc }}^{b}$ \\
\hline \multicolumn{9}{|c|}{ Fit of velocity $\left(\Delta v_{0}=6.3 \mathrm{~km} \mathrm{~s}^{-1}\right)^{c}$} \\
\hline G1 & -0.08 & -0.16 & 8.14 & -0.34 & 164.1 & 554.8 & 2.62 & 1.00 \\
\hline E3 & -0.07 & -0.14 & 8.13 & -0.23 & 163.6 & 554.5 & 2.77 & 1.04 \\
\hline G1b & -0.01 & -0.16 & 8.11 & -0.23 & 162.9 & 560.8 & 2.78 & 1.05 \\
\hline G3 & -0.05 & -0.13 & 8.15 & -0.36 & 164.7 & 554.9 & 2.74 & 1.06 \\
\hline E1 & -0.09 & -0.15 & 8.13 & -0.20 & 163.0 & 554.2 & 2.94 & 1.09 \\
\hline E4 & -0.09 & -0.15 & 8.14 & -0.24 & 163.0 & 554.5 & 3.01 & 1.13 \\
\hline E1b & -0.02 & -0.15 & 8.10 & -0.10 & 161.6 & 560.2 & 3.20 & 1.17 \\
\hline G4 & -0.06 & -0.13 & 8.18 & -0.50 & 164.6 & 554.7 & 3.02 & 1.20 \\
\hline $\mathrm{E} 4 \mathrm{~b}$ & -0.00 & -0.14 & 8.15 & -0.28 & 162.1 & 560.9 & 3.24 & 1.23 \\
\hline $\mathrm{G} 4 \mathrm{~b}$ & -0.00 & -0.15 & 8.18 & -0.47 & 162.5 & 560.7 & 3.19 & 1.24 \\
\hline Gbb & 0.10 & -0.02 & 8.19 & -0.82 & 167.0 & 558.1 & 3.45 & 1.32 \\
\hline Ebb & 0.10 & -0.02 & 8.20 & -0.87 & 166.1 & 558.8 & 3.45 & 1.33 \\
\hline $\mathrm{E} 3 \mathrm{~b}$ & -0.00 & -0.14 & 8.07 & 0.01 & 161.4 & 560.2 & 3.74 & 1.33 \\
\hline average $^{d}$ & $-0.02 \pm 0.06$ & $-0.12 \pm 0.05$ & $8.14 \pm 0.04$ & $-0.36 \pm 0.25$ & $163.6 \pm 1.7$ & $557.5 \pm 2.9$ & & \\
\hline \multicolumn{9}{|c|}{ Fit of velocity and velocity dispersion $\left(\Delta v_{0}=14.3 \mathrm{~km} \mathrm{~s}^{-1}\right)^{c}$} \\
\hline G7 & -0.00 & -0.02 & 8.03 & -0.01 & 167.6 & 549.9 & 10.13 & 0.82 \\
\hline E7 & -0.00 & -0.01 & 7.98 & 0.02 & 167.8 & 548.4 & 10.31 & 0.86 \\
\hline G7b & 0.10 & -0.02 & 8.09 & -0.16 & 168.1 & 560.2 & 10.67 & 0.86 \\
\hline E7b & 0.10 & -0.02 & 8.13 & -0.46 & 167.3 & 560.5 & 10.53 & 0.87 \\
\hline E8 & -0.01 & -0.01 & 7.97 & 0.21 & 163.8 & 548.3 & 11.22 & 0.90 \\
\hline G8 & -0.01 & -0.00 & 8.14 & -0.16 & 164.0 & 551.0 & 11.94 & 0.94 \\
\hline $\mathrm{C} 7$ & -0.00 & -0.01 & 8.01 & 0.02 & 165.3 & 551.0 & 12.81 & 0.94 \\
\hline E5 & -0.01 & -0.00 & 8.11 & -0.03 & 163.4 & 550.5 & 11.96 & 1.00 \\
\hline $\mathrm{G} 3 \mathrm{~b}$ & 0.10 & -0.02 & 8.15 & -0.59 & 166.1 & 562.2 & 7.71 & 1.00 \\
\hline E6 & -0.01 & -0.00 & 8.06 & 0.17 & 161.2 & 549.8 & 12.44 & 1.01 \\
\hline C5 & -0.01 & -0.02 & 8.02 & -0.01 & 167.6 & 550.9 & 13.61 & 1.03 \\
\hline E5b & 0.10 & -0.01 & 8.16 & -0.50 & 164.5 & 562.4 & 12.80 & 1.06 \\
\hline $\mathrm{C} 2 \mathrm{~b}$ & 0.10 & -0.03 & 8.13 & -0.03 & 161.6 & 563.0 & 9.20 & 1.07 \\
\hline $\mathrm{C} 3$ & -0.00 & -0.01 & 8.11 & -0.05 & 162.2 & 553.6 & 9.07 & 1.07 \\
\hline C6b & 0.10 & -0.03 & 8.13 & -0.22 & 161.9 & 563.5 & 12.17 & 1.08 \\
\hline $\mathrm{C} 8 \mathrm{~b}$ & 0.12 & -0.03 & 8.23 & -0.84 & 161.0 & 562.5 & 11.28 & 1.08 \\
\hline $\mathrm{C} 8$ & -0.00 & -0.00 & 8.13 & -0.14 & 162.6 & 552.7 & 13.69 & 1.09 \\
\hline G5 & -0.01 & -0.01 & 8.19 & -0.36 & 162.9 & 550.9 & 13.07 & 1.09 \\
\hline $\mathrm{E} 8 \mathrm{~b}$ & 0.10 & -0.01 & 8.22 & -0.24 & 163.1 & 562.8 & 12.70 & 1.10 \\
\hline G6 & -0.01 & -0.00 & 8.14 & 0.02 & 160.3 & 549.6 & 13.38 & 1.10 \\
\hline G5b & 0.09 & -0.02 & 8.17 & -0.15 & 163.6 & 562.7 & 13.74 & 1.11 \\
\hline G3 & -0.01 & -0.01 & 8.19 & -0.66 & 165.2 & 551.7 & 8.52 & 1.12 \\
\hline $\mathrm{C} 7 \mathrm{~b}$ & 0.14 & -0.04 & 8.09 & -0.20 & 165.5 & 564.7 & 10.26 & 1.13 \\
\hline $\mathrm{C} 3 \mathrm{~b}$ & 0.12 & -0.03 & 8.15 & -0.55 & 165.7 & 562.7 & 8.73 & 1.14 \\
\hline $\mathrm{C} 4$ & -0.01 & -0.00 & 8.14 & -0.17 & 162.9 & 550.6 & 9.74 & 1.16 \\
\hline C6 & -0.01 & -0.01 & 7.96 & 0.17 & 163.6 & 550.5 & 14.57 & 1.17 \\
\hline $\mathrm{C} 1 \mathrm{~b}$ & 0.09 & -0.03 & 8.20 & -0.41 & 161.8 & 562.6 & 9.77 & 1.22 \\
\hline Ecb & 0.10 & -0.01 & 8.10 & -0.22 & 165.5 & 559.3 & 19.74 & 1.25 \\
\hline E3 & -0.01 & -0.00 & 8.20 & -0.92 & 164.5 & 552.0 & 9.86 & 1.27 \\
\hline $\mathrm{Eb}$ & -0.00 & -0.01 & 7.95 & 0.13 & 168.3 & 548.9 & 17.64 & 1.26 \\
\hline Ebb & 0.10 & -0.03 & 8.07 & 0.03 & 166.6 & 562.0 & 18.17 & 1.26 \\
\hline $\mathrm{C} 4 \mathrm{~b}$ & 0.11 & -0.03 & 8.26 & -0.29 & 160.4 & 564.1 & 9.51 & 1.27 \\
\hline E6b & 0.09 & -0.01 & 8.17 & -0.33 & 160.9 & 561.5 & 16.25 & 1.28 \\
\hline average $^{d}$ & $0.01 \pm 0.01^{e}$ & $0.01 \pm 0.01^{e}$ & $8.11 \pm 0.08$ & $-0.21 \pm 0.28$ & $164.1 \pm 2.4$ & $556.3 \pm 6.1$ & & \\
\hline average $^{d}$ & $0.02 \pm 0.01^{f}$ & $-0.10 \pm 0.01^{f}$ & & & & & & \\
\hline
\end{tabular}

${ }^{a}$ Adopted ISBD.

${ }^{b}$ Rescaled $\chi^{2}$ with errors computed as $\Delta v_{i}^{\prime 2}=\Delta v_{i}{ }^{2}+\Delta v_{0}{ }^{2}$.

${ }^{c}$ Systematic error adopted to renormalize $\chi^{2}$.

${ }^{d}$ Average and rms of best fit parameter values.

e Average and rms only on non-"b" ISBDs.

${ }^{f}$ Average and rms only on "b" ISBDs.

^ Parameter was held fixed. 

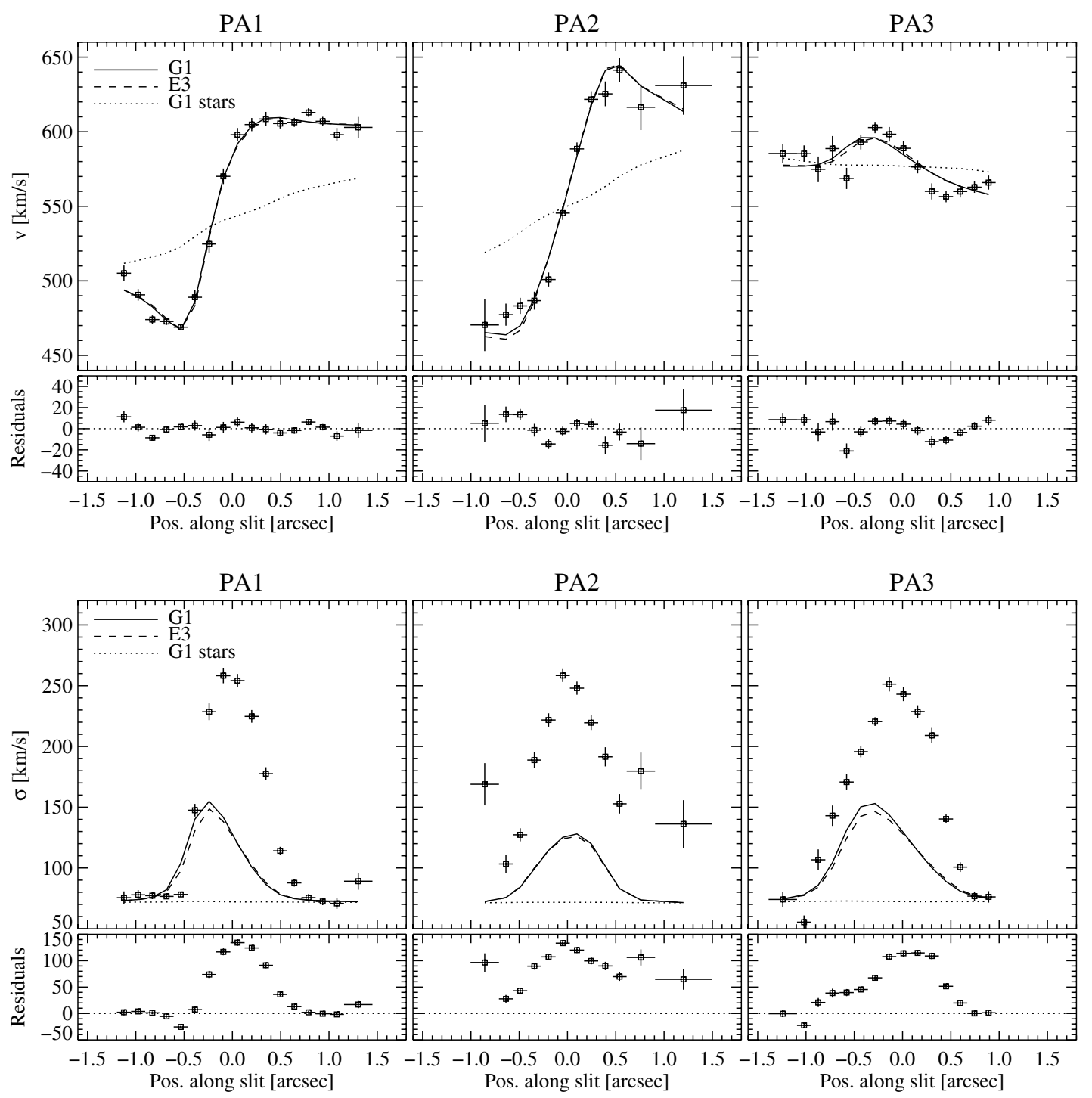

Fig. 20. Top panels: fit of the ISAAC velocities along the slit. The empty squares with error bars represent the observed values while the solid lines connects corresponding model values. Dotted lines represent the contribution of the mass in stars to the rotation curve, i.e. what would be observed without a BH. "G1" and "E3" are the two ISBDs which provide the best fit models with the lowest $\chi^{2}$. Bottom panels: velocity dispersions expected from the two best fit models compared with the observed ones. Same notation as in the upper panel.

show the average values of the fit parameters and the associated rms. It is highly significant that the systematic errors on all parameters and in particular on BH mass are very small. But the most important thing is that whether one fits velocity or velocity and sigma, the BH mass is the same. Indeed $\log M_{\mathrm{BH}}=8.14 \pm 0.04$ (average over different ISBDs, velocity only), $\log M_{\mathrm{BH}}=8.11 \pm 0.08$ (average over different ISBDs, velocity and velocity dispersion). Also all $\theta$ 's are consistent.

In conclusion, for $i=25 \mathrm{deg}$ and ISBD G7, the BH mass estimate is $\log M_{\mathrm{BH}}=8.03 \pm 0.05$ ( \pm 0.06 systematic) and $\theta=$ $165 \pm 3$ ( \pm 1 systematic).

In Fig. 22c and Table 7 we explore how the minimum $\chi^{2}$ varies with varying disk inclination for the models adopting ISBD G7. As for the STIS case, we have renormalized $\chi^{2}$,s so that the model with the minimum $\chi^{2}$ value has $\chi_{\text {red }}^{2}=1$. The ISAAC data clearly favour inclinations $i<35 \mathrm{deg}$ at the $95 \%$ confidence level. This limit on $i$ is smaller than the one derived from STIS data however, given the higher SNR of the ISAAC data and the larger number of data points, we take it as the upper limit on $i$.

\section{Discussion}

In the previous sections we have described in detail the available datasets and the application of the gas kinematical method which provides consistent results from independent analysis of STIS and ISAAC data. In this section we analyze the influence of the ISBD on the final results and we discuss in detail the results obtained using STIS and ISAAC data. We then verify the crucial assumption that the gas is in a thin, circularly rotating disk by comparing observed and model velocity dispersion. We caution about incorrect computations of the model kinematical quantities which might be affected by a subsampling 

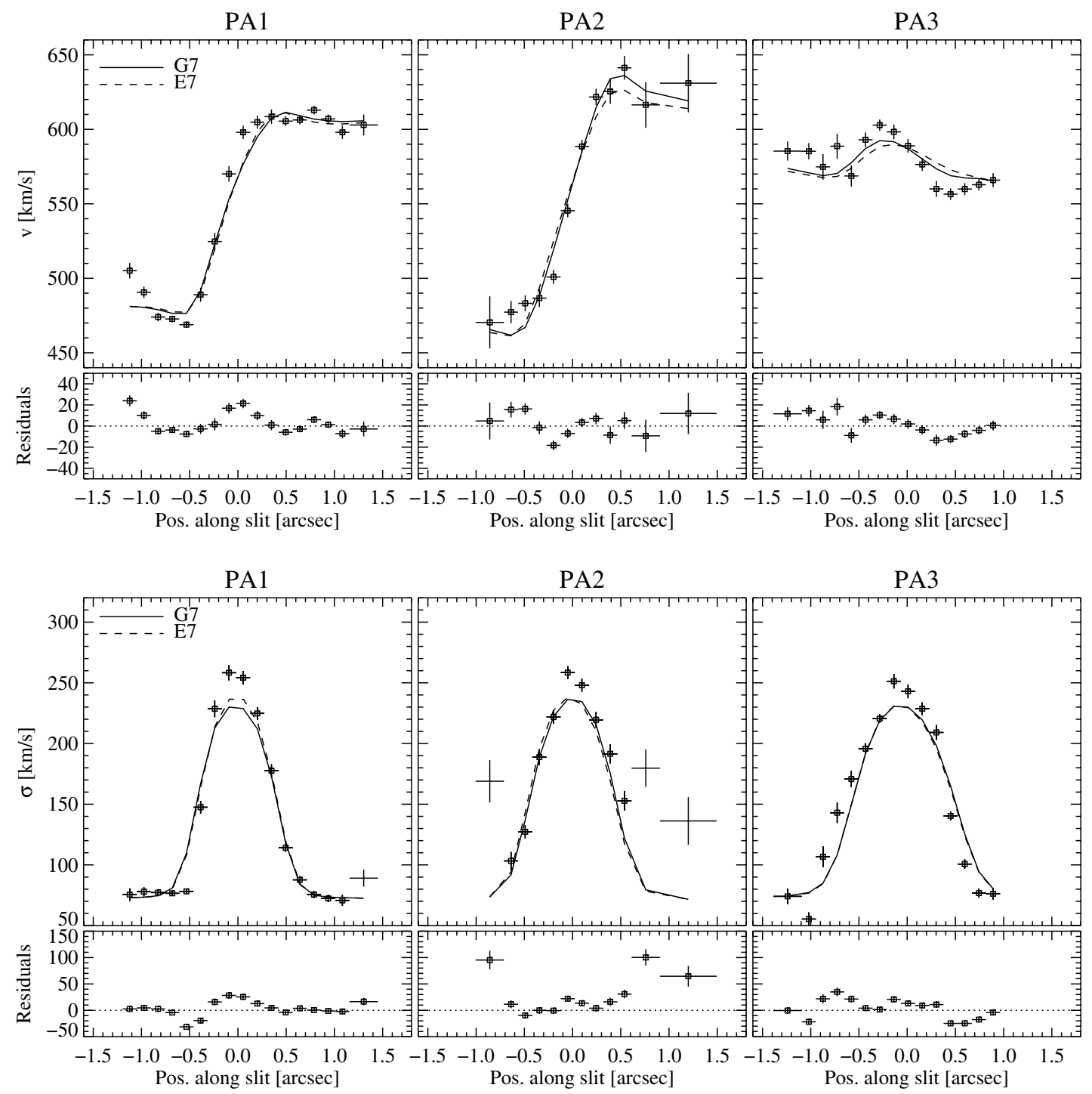

Fig. 21. Fit of the ISAAC velocity and velocity dispersion along the slit. The deviant velocity dispersions in PA1 and PA2 (points not marked with an empty square) were not included in the fit. Notation is the same as in the previous figure.
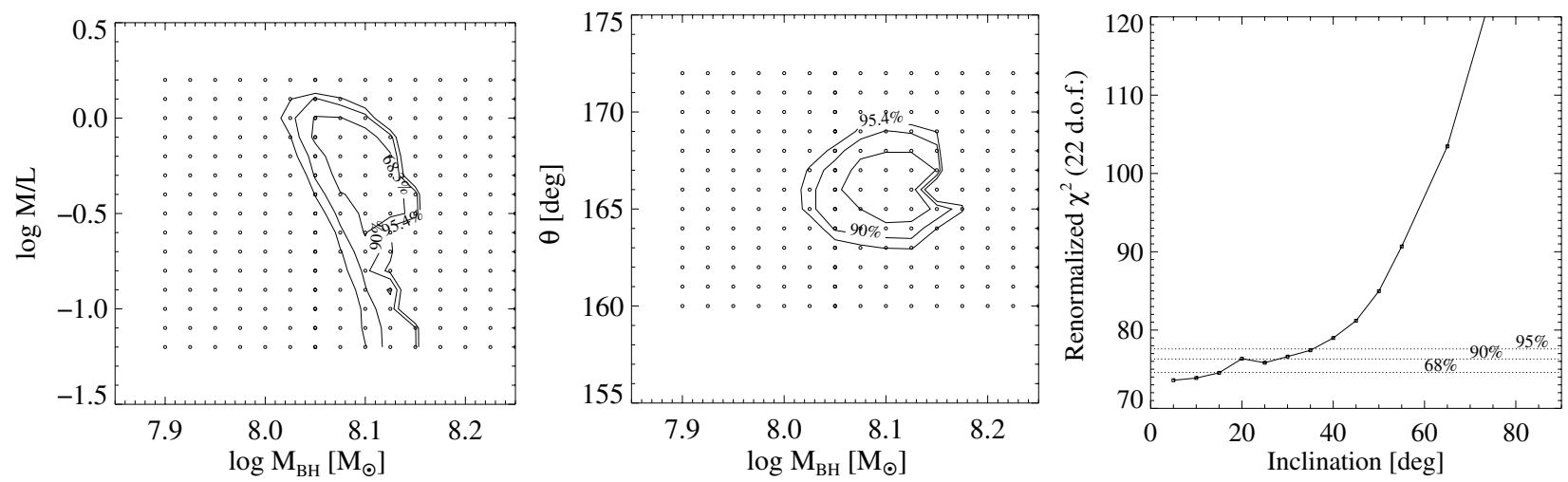

Fig. 22. a) $\chi^{2}$ contours for the joint variation of $M_{\mathrm{BH}}$ and $M / L$ using the ISAAC data and fitting velocities and central velocity dispersions (ISBD G7). Contour levels are for $\chi^{2}=\chi_{\min }^{2}+2.3,4.61,6.17$ corresponding to $68.3 \%, 90 \%$ and $95.4 \%$ confidence levels. The dot indicates the $M_{\mathrm{BH}}$ and $M / L$ values for which the $\chi^{2}$ minimization was computed. b) Same as a) but for $M_{\mathrm{BH}}-\theta$. c) Dependence of $\chi^{2}$ on the adopted disk inclination. Dotted lines indicate the $68.3 \%, 90 \%$ and $95.4 \%$ confidence levels. 
Table 7. Effect of $i$ variation on best fit parameters and minimum $\chi^{2}$ for ISAAC data. All models adopt ISBD G7. Confidence levels $68.3 \%$, $90 \%$ and $95.4 \%$ are at $\chi^{2}=\chi_{\min }^{2}+1.0,2.71,4.0$, for one interesting parameter (Avni 1976). $\chi^{2}$,s have been rescaled as described in the text with $\Delta V_{0}=4.6 \mathrm{~km} \mathrm{~s}^{-1}$.

\begin{tabular}{lcccc}
\hline \hline$i$ & $\log M_{\mathrm{BH}}$ & $\log \left(M_{\mathrm{BH}} \sin ^{2} i\right)$ & $\theta$ & $\chi_{\text {resc }}^{2}$ \\
\hline 5. & 9.38 & 7.26 & 167.0 & 73.6 \\
10. & 8.78 & 7.26 & 167.4 & 73.9 \\
15. & 8.44 & 7.27 & 167.6 & 74.5 \\
20. & 8.20 & 7.27 & 167.6 & 76.3 \\
25. & 8.03 & 7.28 & 167.6 & 75.8 \\
30. & 7.91 & 7.30 & 167.4 & 76.6 \\
35. & 7.81 & 7.33 & 166.2 & 77.4 \\
40. & 7.73 & 7.35 & 167.5 & 79.0 \\
45. & 7.68 & 7.38 & 167.4 & 81.2 \\
50. & 7.65 & 7.42 & 168.7 & 85.0 \\
55. & 7.64 & 7.46 & 168.1 & 90.7 \\
65. & 7.66 & 7.58 & 167.6 & 103.5 \\
75. & 7.98 & 7.95 & 170.1 & 123.6 \\
85. & 8.11 & 8.11 & 175.0 & 226.3 \\
\hline
\end{tabular}

problem (see also Appendix A) or by adopted ISBD which are too smooth. We also compare observed line profiles, $h_{3}$ and $h_{4}$ parameters with model values. Finally, after comparing the gas kinematical estimate of $M_{\mathrm{BH}}$ with the stellar dynamical one by Silge et al. (2005), we discuss $M_{\mathrm{BH}}$ in the framework of the correlations between BH mass and host galaxy structural parameters and we conclude this section by estimating the maximum size of a cluster of dark stellar remnants, the most likely alternative to a supermassive $\mathrm{BH}$.

\subsection{Influence of the intrinsic surface brightness distribution}

One of the worries in gas kinematical measurements of $\mathrm{BH}$ masses has always been the role of the intrinsic emission line surface brightness distribution, since it is the weight of the velocity field in the averaging over apertures (e.g. Macchetto et al. 1997; Barth et al. 2001; Marconi et al. 2003a). In the previous section we have clearly shown that the adopted ISBD, provided that it reproduces the observed one within the errors, does not affect the final $\mathrm{BH}$ mass estimate. Indeed systematic errors on $M_{\mathrm{BH}}$ due to the adopted ISBD are of the order of 0.05 and 0.08 in $\log M_{\mathrm{BH}}$ for STIS and ISAAC data respectively. However it should be remembered that the ISBD has an important effect on the quality of the velocity fit.

We can now compare the results obtained here with those obtained by Marconi et al. (2001) who adopted a simple constant ISBD and a double exponential. From the ISAAC data we obtain $\log M_{\mathrm{BH}}=8.11 \pm 0.08$ (average and rms over all ISBDs) with disk inclination $i=25$. With the same disk inclination, Marconi et al. (2001) obtained $\log M_{\mathrm{BH}}=8.31 \pm 0.1$ (constant ISBD) and $\log M_{\mathrm{BH}}=8.25 \pm 0.1$ (double exponential ISBD). The data are consistent within the errors. The slightly smaller values found here are explained with the fact that Marconi et al. (2001) did not consider the stellar mass in their fitting. Regarding the PA of the disk line of nodes Marconi et al. (2001) found $-15 \pm 5$ while we find $164 \pm 2$, i.e. $-16 \pm 2$. Therefore our new analysis improves the accuracy of the BH mass estimate with respect to Marconi et al. (2001) but does not substantially change their results.

\subsection{Comparison of results from fitting STIS and ISAAC data}

In the previous sections we presented the results of fitting separately STIS and ISAAC data. Since the fits are completely independent and data have spatial resolutions differing by an order of magnitude, the comparison of the results provides an important check of the robustness of the gas kinematical method in measuring $\mathrm{BH}$ masses. Moreover, the comparison will show whether it is possible to obtain an accurate estimate of the BH mass even with lower $\mathrm{S} / \mathrm{N}$ data like those obtained with STIS.

We did not perform a joint fit of the ISAAC and STIS data because this would be strongly biased toward the ISAAC data since the number of points is larger and their relative errors are smaller.

The best fit of STIS data with ISBD G2 (see Table 4) provides $\log \left(M_{\mathrm{BH}} / M_{\odot}\right)=8.00 \pm 0.11$ (statistical) \pm 0.05 (systematic). This is perfectly in agreement with the best fit result of ISAAC data where, from ISBD G7 (Table 6), $\log \left(M_{\mathrm{BH}} / M_{\odot}\right)=8.03 \pm 0.05$ (statistical) \pm 0.08 (systematic) The agreement is still good when comparing the best fit values of the BH mass averaged over all the adopted ISBDs. STIS data provide $\log \left(M_{\mathrm{BH}} / M_{\odot}\right)=7.88 \pm 0.05$ while from ISAAC $\log \left(M_{\mathrm{BH}} / M_{\odot}\right)=8.11 \pm 0.08$. The $\log$ ratio of the $\mathrm{BH}$ masses is $0.2 \pm 0.1$ which is consistent with 0 at the $2 \sigma$ level.

Apart for the $\mathrm{BH}$ mass, the separate fits recover consistent estimates of the position angle of the disk line of nodes. From STIS (G2), the best fit provides $\theta=161 \pm 6$ (statistical) \pm 6 (systematic) while from ISAAC (G7) it results $\theta=168 \pm 2$ (statistical) \pm 2 (systematic). The agreement is even improved when comparing the best fit values of $\theta$ averaged over all the adopted ISBDs: $\theta=168 \pm 6$ (STIS) and $\theta=164 \pm 2$ (ISAAC).

In Fig. 23 we compare the positions of the slit centers and emission line peaks with respect to the kinematical center position derived in the fitting. Since in each fit we leave the coordinates of the kinematical center free to vary, in order to perform the comparison we consider a reference system in which the kinematical center is at $(0,0)$. In figure, slit centers are indicated by empty big symbols while the corresponding positions of the emission line peaks are indicated by smaller filled symbols with the same shape. In the STIS case, the slits were positioned blindly on the nucleus position which was computed from NICMOS K images comparing the location on the continuum peak with respect to the acquisition star. The ISAAC slits were directly centered on the location of the $K$ continuum peak. During the fitting of the ISAAC data we considered two cases, one where the emission line peak was coincident with the slit centers (i.e. with the $K$ continuum peak as suggested by the NICMOS Pa $\alpha$ image), and the other, labeled "b", where the 


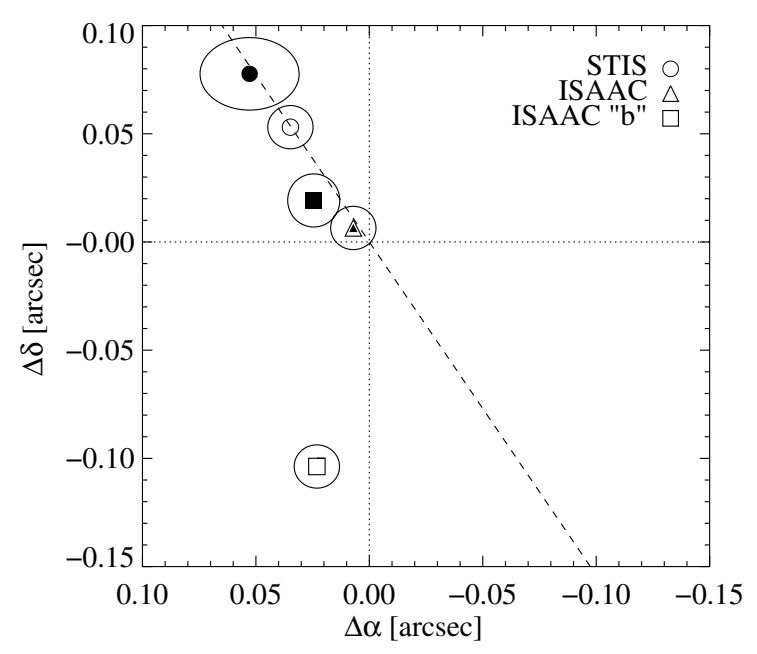

Fig. 23. Positions of slit centers (empty symbols) and emission line peaks (filled symbols) compared to the kinematical center located at $\Delta \alpha=0, \Delta \delta=0$. Ellipses indicate the $1 \sigma$ errors. The dashed line is the $\mathrm{PA}$ of the jet observed at radio and X-ray wavelengths.

line emission peak was not coincident with the slit centers. In all cases slit centers are closer than 0.1 arcsec from the kinematical center. But the most interesting indication comes from the location of the emission line peaks which are, within the errors, located along the jet axis, a result not obvious a-priori. This is consistent with two possible pictures: in the first one the jet excites line emission while in the second one the jet represents the symmetry axis of the ionization cone, according to the unified model of AGNs (e.g. Axon et al. 1998; Capetti et al. 1999).

\subsection{Is the gas circularly rotating? Velocity dispersion and line profiles tests.}

The power of gas kinematical measurements is their conceptual simplicity compared to the complex analysis required by stellar dynamical modeling for which there are serious issues regarding indeterminacy (Valluri et al. 2004; Cretton \& Emsellem 2004). However, gas kinematical measurements are based on the assumption of Keplerian disks, i.e. thin disks circularly rotating under the influence of the gravitational potential due to stars and BH. In some cases the gas is clearly not rotating circularly, for instance when the motions are affected by jet-cloud interactions (e.g. Axon et al. 1998; Capetti et al. 1999) but in other cases the question is more subtle.

The comparison between observed and model velocity dispersion has become one of the most used tests on the reliability of the circular rotation assumption: velocity dispersions larger than expected from unresolved rotation could be an indication of non circular motions which could invalidate the $\mathrm{BH}$ mass estimate. Many authors have found that the observed velocities could be well fit with Keplerian disk models but the gas velocity dispersion was larger, and in some cases much larger than expected from unresolved rotation. This has caused big concerns on the reliability of the gas kinematical method (e.g. van der Marel \& van den Bosch 1998; Barth et al. 2001; Verdoes Kleijn et al. 2000, 2002; Cappellari et al. 2002).
Before focussing on Centaurus A, we show that these concerns might have been excessive since a small model velocity dispersion could easily result from (i) a non-accurate computation or, more likely, from (ii) an adopted ISBD which is too smooth.

In Appendix A we show the effect of a coarse sampling in the computation of the model kinematical quantities. This effect is particularly important when the ISBD varies on spatial scales which are much smaller than the PSF sizes and Fig. A.1 shows how one could easily underestimate the model velocity dispersion by a factor $\simeq 1.5$. This effect is also very subtle because it is not manifested in the model computed line fluxes and velocities. We thus caution about the sampling chosen in computations and in Appendix A we present a possible way to overcome this potential problem.

We now focus on the effects of different ISBs which provide the same light profile along the slit after being convolved with the instrumental response. We consider three different ISBs labeled A, B and C. All of them are circularly symmetric on the plane of the sky and are described by a sum of two exponentials as

$f(r)=I_{0} \mathrm{e}^{-r / r_{0}}+I_{1} \mathrm{e}^{-r / r_{1}}$.

ISBD A has $I_{1} / I_{0}=0.01, r_{0}=0.05^{\prime \prime}$, and $r_{1}=1.00^{\prime \prime}$. B has $I_{1} / I_{0}=0.0067, r_{0}=0.08^{\prime \prime}$ but the second exponential is substituted with a constant. Finally $\mathrm{C}$ has $I_{1} / I_{0}=0.0008$, $r_{0}=0.01^{\prime \prime}$, and $r_{1}=0.69^{\prime \prime}$. The "observed" system is like Centaurus A but with $M_{\mathrm{BH}}=10^{8} M_{\odot}, M / L=1$, and the gas disk is inclined by $45 \mathrm{deg}$ with respect to the line of sight. The rotating gas disk, which has an intrinsic constant velocity dispersion of $70 \mathrm{~km} \mathrm{~s}^{-1}$, is observed with ISAAC with $0.5^{\prime \prime}$ seeing. The slit is placed along the major axis of the disk. In Fig. 24 we show the intrument convolved surface brigtness, velocity and velocity dispersion along the slit. As noted in previous sections, it is clear that the observed surface brightness and velocity along the slit (and consequently $M_{\mathrm{BH}}$ ) are little sensitive to the choice of the ISBD. The reason for this weak dependence of the instrument convolved velocity on the ISBD is that, close to the BH location where the weighting by the ISBD is larger, rotation is not resolved and the contribution from positive and negative velocities is canceled. However, the effects of the ISBD are readily apparent on the gas velocity dispersion. Suppose that in the analysis of the data one uses ISBD A and finds that, while the observed velocities are well reproduced by the model, the gas velocity dispersion is underestimated by a factor $\simeq 2$. One might then be tempted to conclude that the gas has a high intrinsic velocity dispersion. However, using ISBD C (or even a less extreme one) one would reach the opposite conclusion regarding the validity of the Keplerian disk assumption.

It is not possible to draw general conclusions here because each case must be analyzed separately bearing in mind the possibility that the adopted ISBD does not reflect the real one. However we caution against the possibility that many of the previous gas kinematical analyses finding inconsistent gas velocity dispersions might be affected either by a subsmapling problem or by the adoption of a too extended ISBD.

We now focus on Centaurus A where the STIS and ISAAC datasets are well suited to this discussion. In the case of 

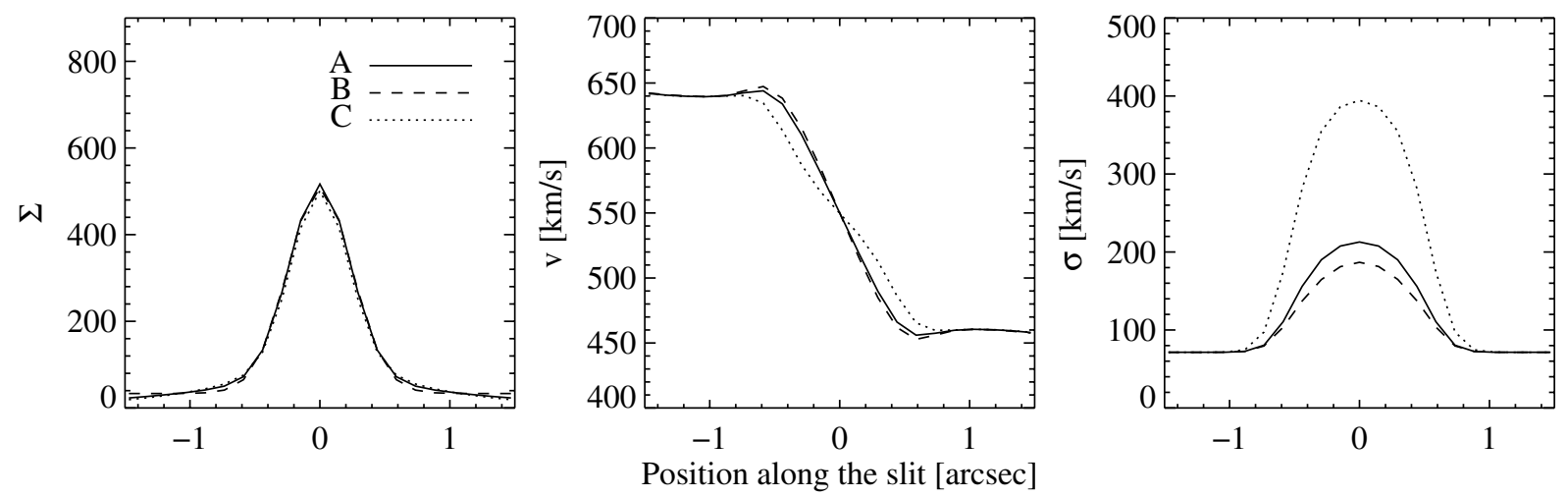

Fig. 24. Instrument convolved kinematical quantities computed with different ISBDs A, B and C (see text for explanation). The ISBDs have been chosen to provide very similar observed surface brightnesses along the slit.
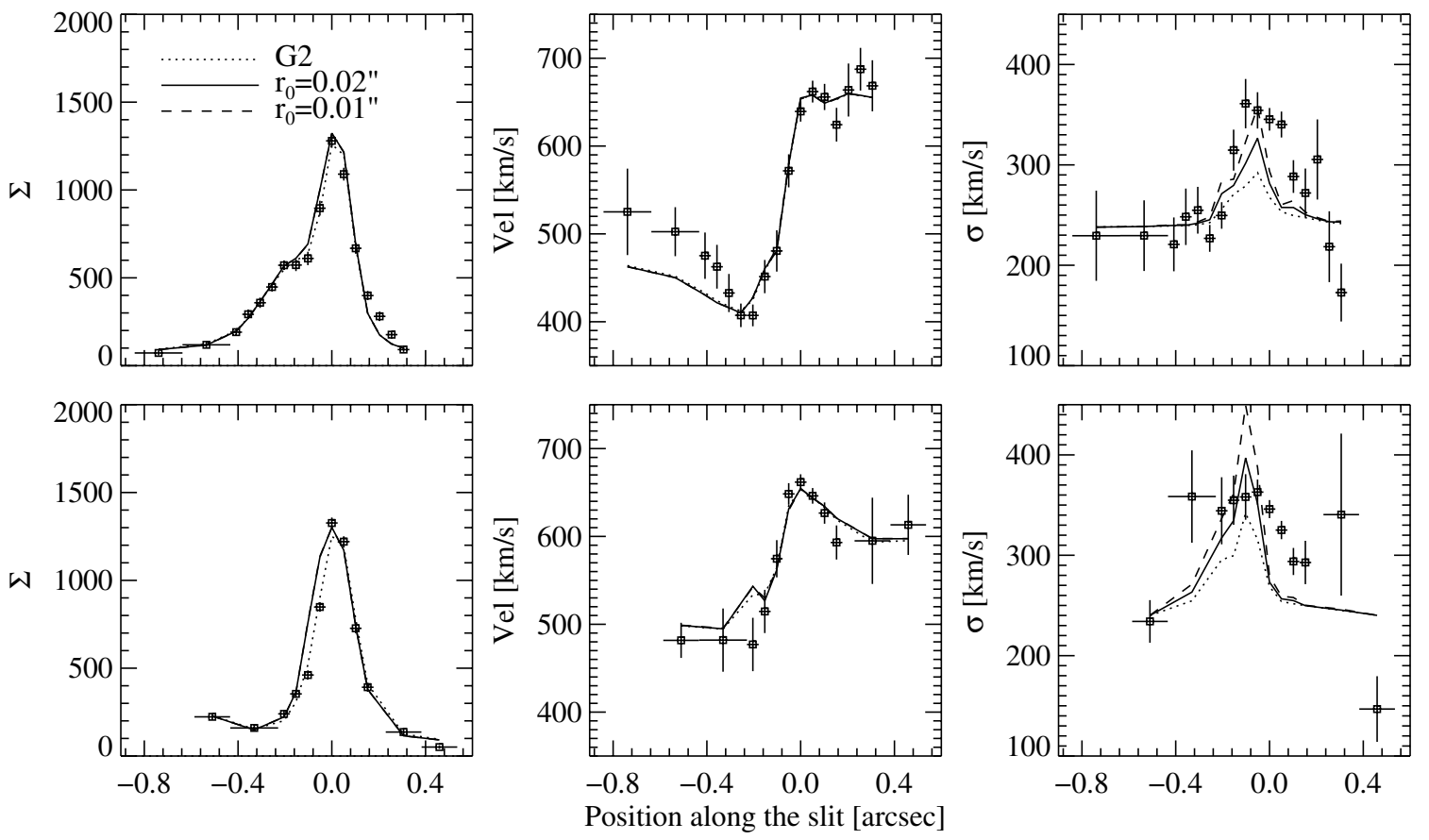

Fig. 25. Comparison between observed kinematical quantities from STIS (points with error bars) and model computations. From left to right panels represent surface brightness, velocity and velocity dispersion along the slit. The top and bottom panels are for VIS1 and VIS2, respectively. Models were computed using G2 and two additional ISBD contructed by adding to G2 an exponential component with $r_{0}=0.02^{\prime \prime}$ and $r_{0}=0.01^{\prime \prime}$ at the position of the BH (see text for more details).

ISAAC data, the model fitting of velocities does not reproduce the velocity dispersion and the reason is that, in order to find the best match of the rotation curves, the kinematical center is pushed away from the emission line peak. However, the joint fit of velocity and velocity dispersion shows that the circularly rotating model can well account for both velocity and velocity dispersion at the same time (note that the $M_{\mathrm{BH}}$ estimate is not changed by this).

The observed velocity dispersion in the STIS datasets is larger than expected from pure rotation but the previous discussion on the effects of the ISBD on the model velocity dispersion might suggest that it is possible to match the observed STIS velocity dispersion with an appropriate choice of the ISBD. However the main ISBD component used for STIS is not located on the $\mathrm{BH}$ but $\simeq 0$.' 1 away, therefore changing its scale radius would not produce any appreciable effect on final model values. Instead, one should place a bright and very peaked ISBD component on the BH location. In Fig. 25 we compare model kinematical quantities obtained using G2 and two additional ISBDs obtained by adding an exponential component to $\mathrm{G} 2$ at the location of the BH. These exponential components have $I_{0}=5000, r_{0}=0.02^{\prime \prime}$ and $I_{0}=20000, r_{0}=0.01^{\prime \prime}$ respectively (see also Table 2 for the parameters describing G2). Surface brightnesses and velocities are very little affected by this additional ISBD component located at the position of the $\mathrm{BH}$. However the gas velocity dispersion is increased and it is now possible to reproduce the peak value of the observed velocity dispersion. The observed $\sigma$ profile along the slit is slightly wider than the model one nonetheless, given the signalto-noise ratio of the data, the agreement between models and 
Flux distribution G7

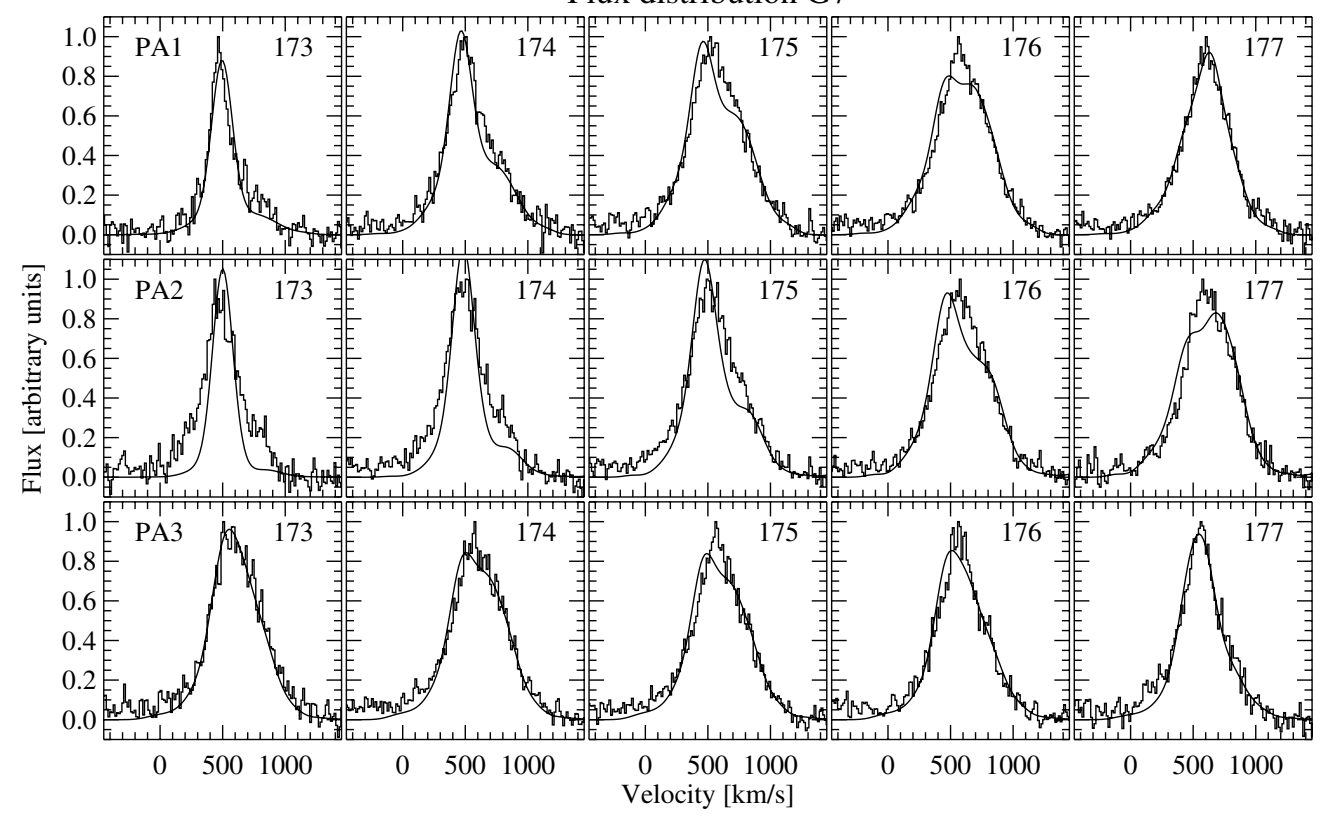

Flux distribution E5

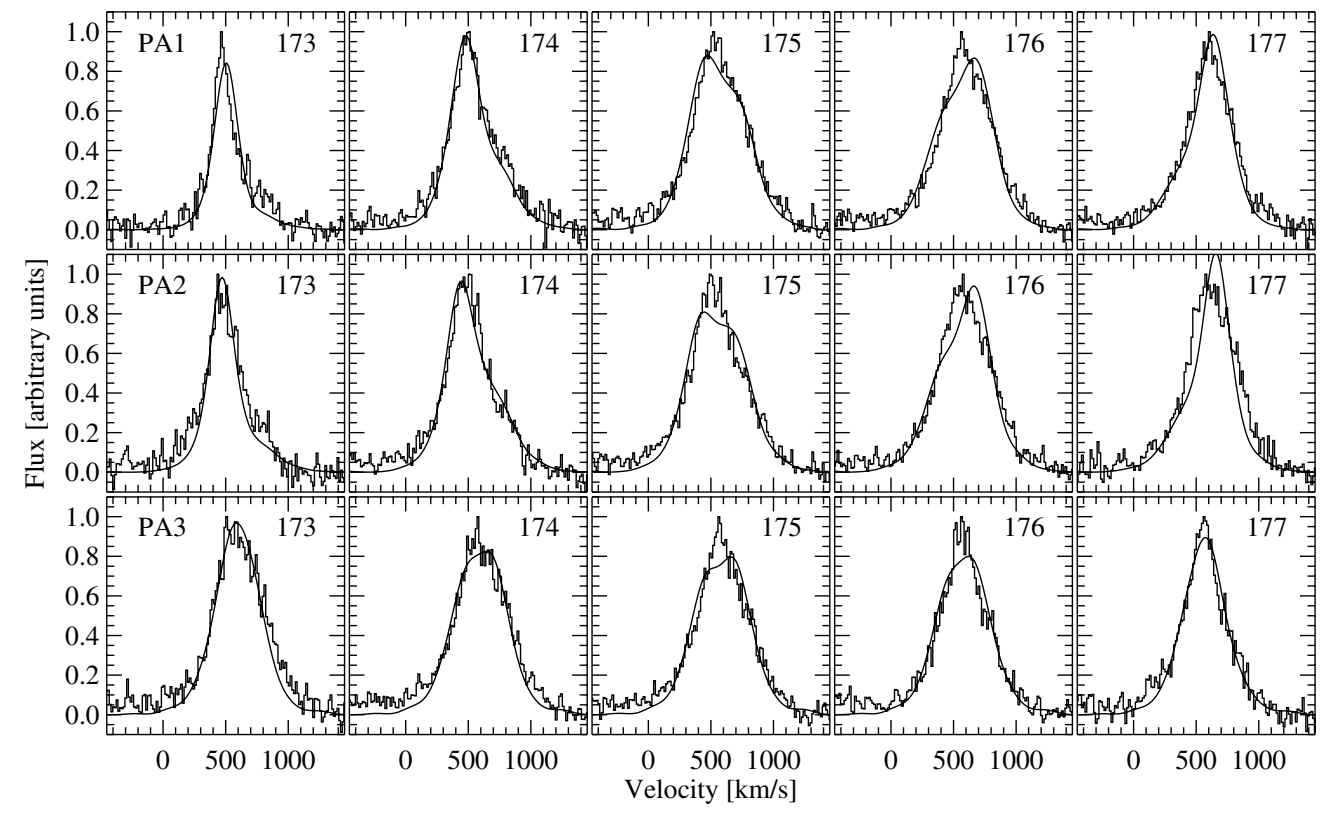

Fig. 26. Top panels: observed ISAAC line profiles are compared with model ones computed with the best ISBD G7. Numbers on the top right corners indicate the row where the spectrum was extracted (175 is the location of the slit center). Model profiles have been rescaled in surface brightness to have the best match with observed ones. Bottom: as in top panels, but for ISBD E5 which provides the best matching model line profiles.

observations can now be considered satisfactory. The intrinsic constant velocity dispersion required by the data, $\sigma_{0}=$ $160 \mathrm{~km} \mathrm{~s}^{-1}$, is smaller than that expected from circular rotation and rotation around a $\mathrm{BH}$ is needed to explain the increase of $\sigma$ in the nuclear region. The measured $M_{\mathrm{BH}}$ is perfectly consistent with the results from the ISAAC dataset. This is a clear indication that even the presence of a constant intrinsic velocity dispersion $\left(\sigma_{0}=160 \mathrm{~km} \mathrm{~s}^{-1}\right)$ does not necessarily invalidate the $M_{\mathrm{BH}}$ estimate. In conclusion it is important to emphasize that a large velocity dispersion rise in the nucleus of a galaxy cannot be a manifestation of the ISBD in a pure stellar model.
Thus their very existence is prima facie evidence for the presence of a $\mathrm{BH}$ provided the gas motions are dominated by rotation. However, due to the sensitivity of the observed sigma to the ISBD, modeling a sigma peak is model dependent and therefore results in weak constraints on the precise mass of the $\mathrm{BH}$.

In Fig. 26 we compare the model line profiles computed with ISBD G7 with the observed ones with ISAAC. Such a comparison is meaningless with STIS because of the much lower spectral resolution. The match between observed and model line profiles is good given that the fitting was performed 
on velocity and velocity dispersion and not on the line profile itself. G7 is the ISBD which provides the best fit of velocity and velocity dispersion. We have also searched for the ISBD which provides the best match of the line profiles among those in Table 6. A $\chi^{2}$ analysis indicates that this is given by E5 and indeed also a visual inspection of the figure confirms this result. Model line profiles, which are only affected by unresolved rotation, are able to reproduce, at least qualitatively, the features which are observed in the data like asymmetries and humps (see also the discussion in Macchetto et al. 1997). It is thus clear that line profiles are consistent with the rotating disk model and that it is possible to discriminate among different ISBD on the basis of the line profiles.

In this paper we have, for the first time, introduced the Hermite expansion in the analysis of emission line profiles. It is therefore natural to verify whether the model values of $h_{3}$ and $h_{4}$ are consistent with observed ones. In Fig. 27 we perform this comparison using the best fitting ISBD G7. The agreement is not very good but it must be kept in mind that the fit was not optimized to reproduce $h_{3}$ and $h_{4}$. Qualitatively the model reproduces the shapes of the $h_{3}$ and $h_{4}$ variations along the slit, though it does not reproduce the amplitudes. As in the previous case, we have searched for the best fit model which provides the best match to $h_{3}$ and $h_{4}$ and this results to be that generated with ISBD E8. The match is much better than previously though not yet as good as the match of the observed velocity and velocity dispersion. As in the previous case we can conclude that the comparison with $h_{3}$ and $h_{4}$ can be used to discriminate among different ISBDs but this is beyond the scope of this paper.

\subsection{Comparison with stellar dynamical measurements}

Silge et al. (2005) very recently presented a stellar dynamical measurement of the BH mass in Centaurus A. They use axisymmetric 3-integral stellar dynamical models based on the orbit superposition method by Schwarzschild. They estimate a BH mass of $2.4_{-0.2}^{+0.3} \times 10^{8} M_{\odot}$ for edge-on models (i.e. where the principal plane of the potential is edge-on, $i=90 \mathrm{deg}$, with respect to the line of sight), $1.8_{-0.4}^{+0.4} \times 10^{8} M_{\odot}$ for $i=45 \mathrm{deg}$, and $1.5_{-0.2}^{+0.3} \times 10^{8} M_{\odot}$ for $i=20 \mathrm{deg}$. In the Keplerian disk approximation, the gas disk is rotating in the galaxy principal plane. Therefore the inclination of the principal plane is directly the inclination of the gas disk. Thus, in Table 8 we compare gas kinematics and stellar dynamical BH mass measurements computed with the same inclination.

It is readily apparent that there is an excellent agreement between the two measurements if we consider the $i=20 \mathrm{deg}$ case while, at intermediate inclination $i=45 \mathrm{deg}$, the measurements are discrepant by at least a factor of 2 . The good agreement for $i=90 \mathrm{deg}$ is not significant because the $\chi^{2}$ in the gas kinematical analysis is a factor of 3 worse than at other inclinations. As discussed in the previous sections, gas kinematics favour low inclinations of the gas disk and, indeed, the model at $i=20$ deg has the lowest $\chi^{2}$ among those in the table. On the contrary, stellar dynamical models suggest an edge on principal plane which is completely excluded by gas kinematical models.
The stellar dynamical measurements are potentially affected by several problems, first of all, as discussed in Silge et al. (2005), the possibility that Centaurus A is not axisymmetric. Centaurus A has a spherical simmetry in the nuclear region but it seems moderately triaxial in the outer regions. At variance with gas kinematics which can work only with the rotation curves from the nuclear region, stellar dynamics needs extended data to constrain stellar orbits (e.g. Valluri et al. 2004; Cretton \& Emsellem 2004). As a consequence, Silge et al. (2005) conclude that it is unlikely that they can constrain the actual inclination of Centaurus A using an axisymmetric modeling procedure.

Moreover, the inclination of the gas disk is not necessarily the same as that of the galaxy principal plane. In the region where we obtain the gas kinematics the gravitational potential is completely dominated by the $\mathrm{BH}$ and the gas might lie in a plane which is not coincident with the principal plane of the galaxy.

The above considerations allow us to relax the constraint on having the same inclination for gas and stellar kinematical models and we can thus conclude that the two methods provide results in excellent agreement. This is the first time, for an external galaxy, that there are consistent $\mathrm{BH}$ mass measurements with gas and stellar dynamics.

This agreement should not be surprising since it is found even for the closest supermassive $\mathrm{BH}$, that in the Galactic Center. Notwithstanding the complex environment, gas kinematical measurements from Genzel \& Townes (1987) provide a $M_{\mathrm{BH}}$ measurement which is in excellent agreement with that of the stars (see, e.g., Fig. 11 of Schödel et al. 2003). The only problem of the gas is that it is not present in the inner $0.1 \mathrm{pc}$ and therefore the constraints it poses on the size of the massive dark object are much weaker that those of the stars which can probe the gravitational potential more than 2 orders of magnitude closer to the location of Sgr A*.

\subsection{Correlations with galaxy properties}

The BH mass estimate in Centaurus A can be summarized as $M_{\mathrm{BH}}=(1.1 \pm 0.1) \times 10^{8} M_{\odot}$ for an assumed disk inclination of $i=25 \mathrm{deg}$ or $M_{\mathrm{BH}}=(6.5 \pm 0.7) \times 10^{7} M_{\odot}$ for $i=35 \mathrm{deg}$. We now compare these two values with the known correlations with host spheroid luminosity and mass (we consider for the analysis the results by Marconi \& Hunt 2003) and with the stellar velocity dispersion (Tremaine et al. 2002; Ferrarese \& Ford 2005).

We use the $K$ band $M_{\mathrm{BH}}-L_{\mathrm{sph}}$ correlation by Marconi \& Hunt (2003) who consider only galaxies with "secure" $M_{\mathrm{BH}}$ determination. The $K$ band total luminosity of Centaurus A is estimated by Marconi \& Hunt (2003) as $M_{K}=-24.5$ ( $\left.L_{K}=1.3 \times 10^{11} L_{\odot, K}\right)$ and the $\mathrm{BH}$ mass expected from the correlation is $\sim 2.8 \times 10^{8} M_{\odot}$, in agreement with our results if one takes into account observational errors and the intrinsic scatter of the $M_{\mathrm{BH}}-L_{\mathrm{sph}}$ correlation which is 0.3 in $\log M_{\mathrm{BH}}$ (a factor of 2). Marconi \& Hunt (2003) also found that the virial mass $\left[M_{\mathrm{sph}}=3 R_{\mathrm{e}} \sigma_{\mathrm{e}}^{2} / G\right]$ is correlated with $M_{\mathrm{BH}}$. Using their estimate of $M_{\text {sph }}$ for Centaurus A $\left(M_{\text {sph }}=(5.6 \pm 1.5) \times 10^{10} M_{\odot}\right)$ 


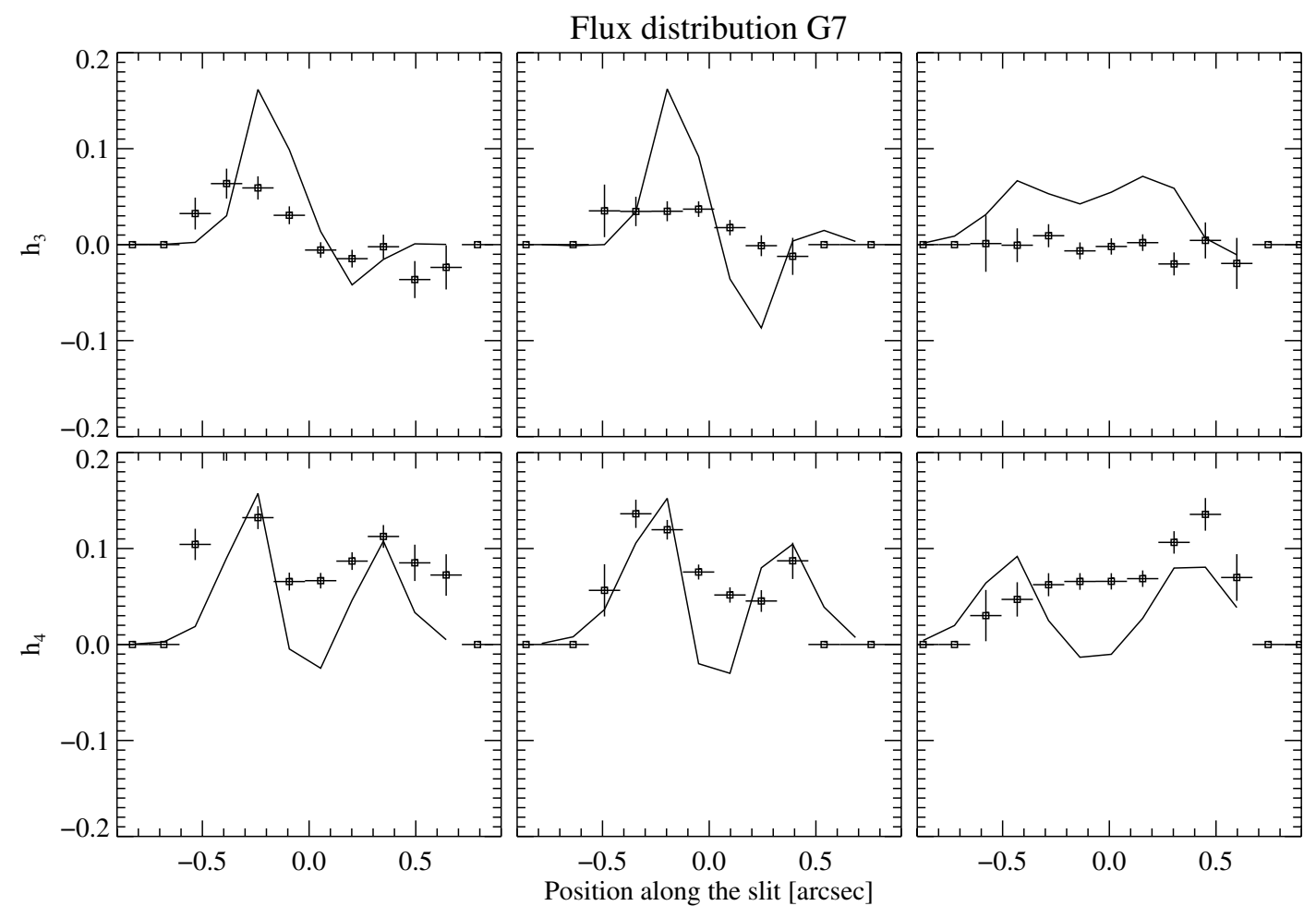

Flux distribution E8
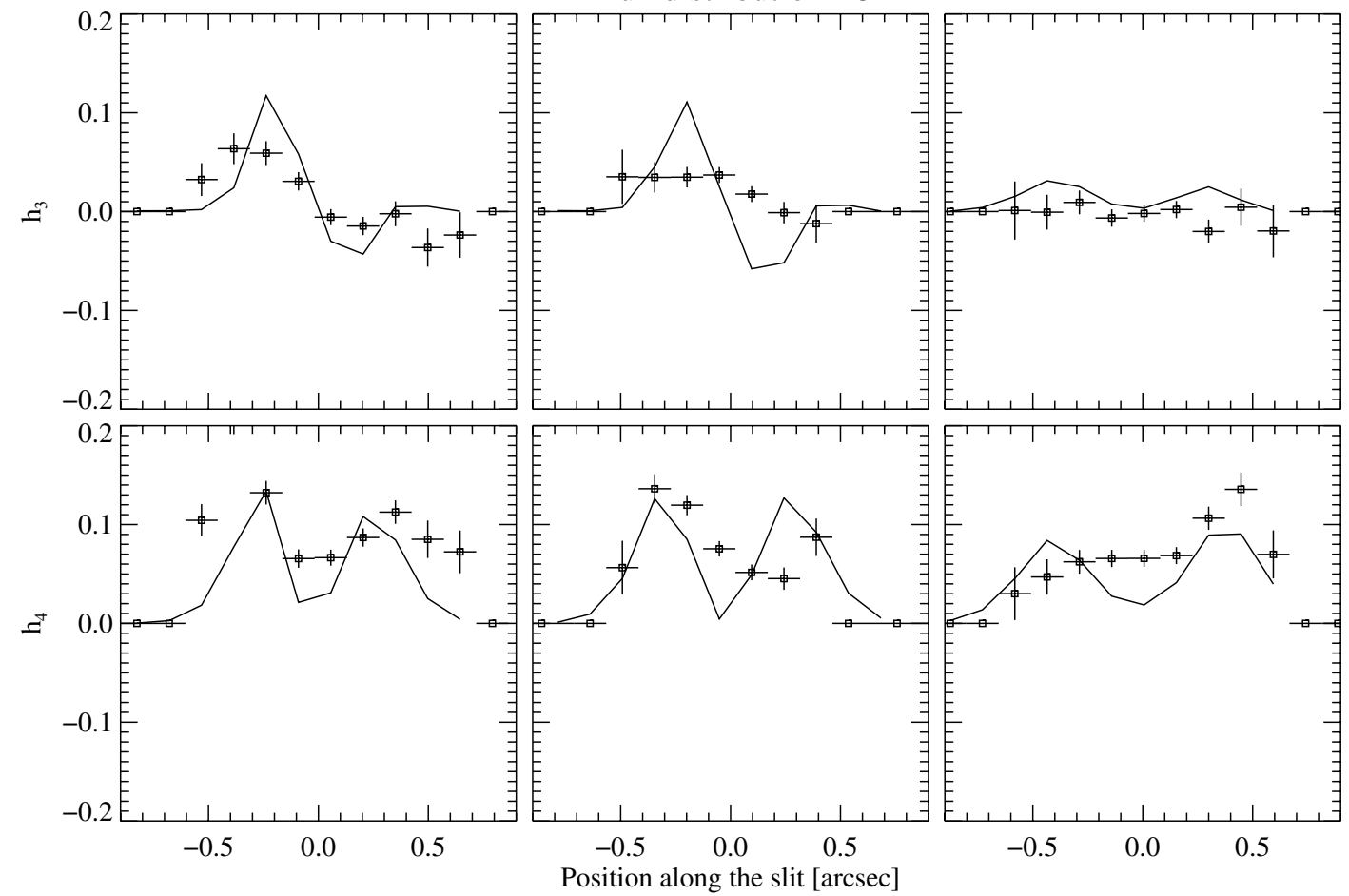

Fig. 27. Top panels: observed Hermite parameters $h_{3}$ and $h_{4}$ compared with model ones computed with the best ISBD G7. Bottom: as in top panels, but for ISBD E8 which provides the best match to the observations.

and the correlation which considers only galaxies with "secure" $M_{\mathrm{BH}}$ determination, the expected BH mass for Centaurus A is $\sim 1.4 \times 10^{8} M_{\odot}$, again in excellent agreement with our measurement. Therefore we can conclude that $M_{\mathrm{BH}}$ in Centaurus A is in very good agreement with $L_{\mathrm{sph}}$ and $M_{\mathrm{sph}}$.
The luminosity weighted stellar velocity dispersion of Centaurus A using an aperture of $60^{\prime \prime}$ parallel to the dust lane is $\sigma=138 \pm 10 \mathrm{~km} \mathrm{~s}^{-1}$ (Silge et al. 2005). Assuming that this is a good approximation for $\sigma_{\mathrm{e}}$ (the same quantity but averaged in an aperture $R_{\mathrm{e}}$, the galaxy effective radius), we can 
Table 8. Comparison of BH mass measurements with gas kinematics and stellar dynamics.

\begin{tabular}{lllll}
\hline \hline$i$ & \multicolumn{2}{c}{ Gas kinematics $^{a}$} & \multicolumn{2}{c}{ Stellar dynamics } \\
& $M_{\mathrm{BH}}$ & $\chi^{2} /$ d.o.f. $\left.{ }^{a}\right)$ & $M_{\mathrm{BH}}$ & $\chi^{2}$ d.o.f. \\
\hline 20 & $1.9_{-0.2}^{+0.2} \times 10^{8}$ & $41 / 41$ & $1.5_{-0.2}^{+0.3} \times 10^{8}$ & $\ldots$ \\
45 & $4.8_{-0.5}^{+0.6} \times 10^{7}$ & $44 / 41$ & $1.8_{-0.4}^{+0.4} \times 10^{8}$ & $\ldots$ \\
$90^{b}$ & $3.8_{-0.4}^{+0.5} \times 10^{8}$ & $130 / 41$ & $2.4_{-0.2}^{+0.3} \times 10^{8}$ & $\ldots$ \\
\hline
\end{tabular}

${ }^{a}$ Rescaled $\chi^{2}$ from Table 7 .

${ }^{b}$ For gas kinematics $i=85 \mathrm{deg}$.

estimate the expected $\mathrm{BH}$ mass according to the $M_{\mathrm{BH}}-\sigma_{\mathrm{e}}$ correlation. Adopting the correlation parameters estimated by Tremaine et al. (2002) the expected BH mass is $\sim 3.0 \times 10^{7} M_{\odot}$ while with the version of the correlation by Ferrarese \& Ford (2005) $M_{\mathrm{BH}} \sim 3.0 \times 10^{7} M_{\odot}$. The $\mathrm{BH}$ in Centaurus A is thus, apparently, a factor $2-4$ more massive than expected from the correlations. However, if the scatter of the intrinsic scatter of the $M_{\mathrm{BH}}-\sigma_{\mathrm{e}}$ correlation is taken into account $\left(0.3\right.$ in $\left.\log M_{\mathrm{BH}}\right)$, then the discrepancy can be fully accounted for. In conclusion, if the $M_{\mathrm{BH}}-\sigma_{\mathrm{e}}$ is not a perfect correlation, like it is reasonable to expect, then the $\mathrm{BH}$ in Centaurus $\mathrm{A}$ is not a deviant point.

\subsection{The size of the dark cluster}

The kinematical analysis which leads to the discovery of a BH and measurement of its mass in reality produces evidence only for the existence of a massive dark object. The size of this object is usually constrained to be smaller that the spatial resolution of the observations but in most cases, except for our own Galactic Center (e.g. Schödel et al. 2003), this is not enough to exclude that the massive dark object is not a cluster of dark objects.

In the present paper we can take advantage of the high spatial resolution achieved with HST to estimate a tight upper limit to the size of the putative dark cluster. We assume that the dark mass is distributed according to a Plummer model (e.g. Binney \& Tremaine 1987) and we explore the $\chi^{2}$ variation for fixed values of the core radius, $R_{\text {core }}$, minimizing all the other free parameters of the fit $x_{0}, y_{0}, \log M_{\text {core }}$ (core mass), $\theta, v_{\text {sys. }}$. Results, using ISBD G2 and $i=25 \mathrm{deg}$, are shown in Fig. 28. As before, $\chi^{2}$ is rescaled in order to have $\chi_{\text {red }}^{2}=1$ for the best fitting model and confidence levels are computed following Avni (1976). To the 90\% confidence level, the core radius is less than $0{ }^{\prime} 036(0.6 \mathrm{pc})$. This value increases to $0{ }^{\prime} 04$ $(0.7 \mathrm{pc})$ for a disk inclination of $i=35 \mathrm{deg}$. This implies that the putative massive dark cluster must have a minimum density of $\rho_{\mathrm{BH}}=M_{\mathrm{BH}} /\left(4 / 3 \pi R_{\text {core }}^{3}\right)=1.1 \times 10^{8} M_{\odot} \mathrm{pc}^{-3}$. This value is almost a factor 6 larger than that found for M 87 which, after our own galaxy and NGC 4258, was among the best cases for a BH. Maoz (1998) computed the lifetimes of massive dark clusters with given mass and density before their collapse to a supermassive $\mathrm{BH}$. This lifetime, if much shorter than the age of the universe, indicates that the most likely alternative is that of a BH. Figure 29 is an update of Fig. 1 by Maoz (1998) with the compilation by Marconi \& Hunt (2003). The stars mark the

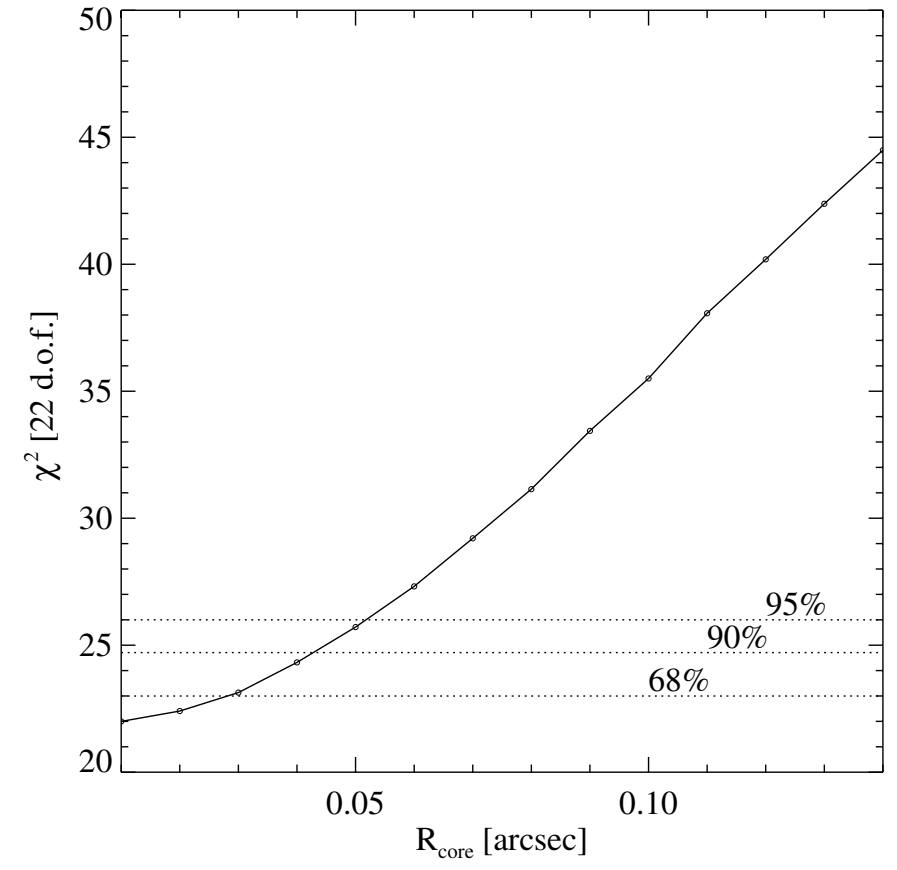

Fig. 28. Maximum core radius from STIS observations (ISBD G2 and $i=25$ ).

new location of Centaurus A based on HST STIS observations for inclination $i=25$ and $35 \mathrm{deg}$. For comparison, we also show the previous location of Centaurus A based on ground based ISAAC observations only. The solid lines are the loci of constant cluster lifetimes computed by Maoz (1998). The lifetime of the putative dark cluster in Centaurus A is still larger than $10^{11} \mathrm{yr}$ and comparable to the age of the universe indicating that a cluster of dark objects cannot be ruled out. Still, Centaurus A ranks now among the best cases for a $\mathrm{BH}$ indicating that much work is still waiting to secure BH mass determinations and the proof on the existence of supermassive BHs. To improve in this direction one needs spatial resolution of the order of 1 mas such as those which can be reached with new generation interferometers like the VLTI (e.g. Marconi et al. 2003b).

\section{Summary and conclusions}

We have presented new HST Space Telescope Imaging Spectrograph observations of the nearby radio galaxy NGC 5128 (Centaurus A). Since the galaxy nucleus is reddened by $A_{\mathrm{V}}>7 \mathrm{mag}$, we used the the redder emission line accessible from HST, [S III] $\lambda 9533 \AA$, to study the kinematics of the ionized gas at 0 '. 1 spatial resolution.

The STIS data were analized in conjunction with the ground-based near-infrared Very Large Telescope ISAAC spectra used by Marconi et al. (2001) to infer the presence of a supermassive black hole and measure its mass. The ISAAC data were re-analyzed and we introduced the use of the Hermite expansion for the kinematical analysis of emission lines. The Hermite expansion allows an excellent fit of the observed line profiles with a smaller number of parameters and more stability than two Gaussian functions. 


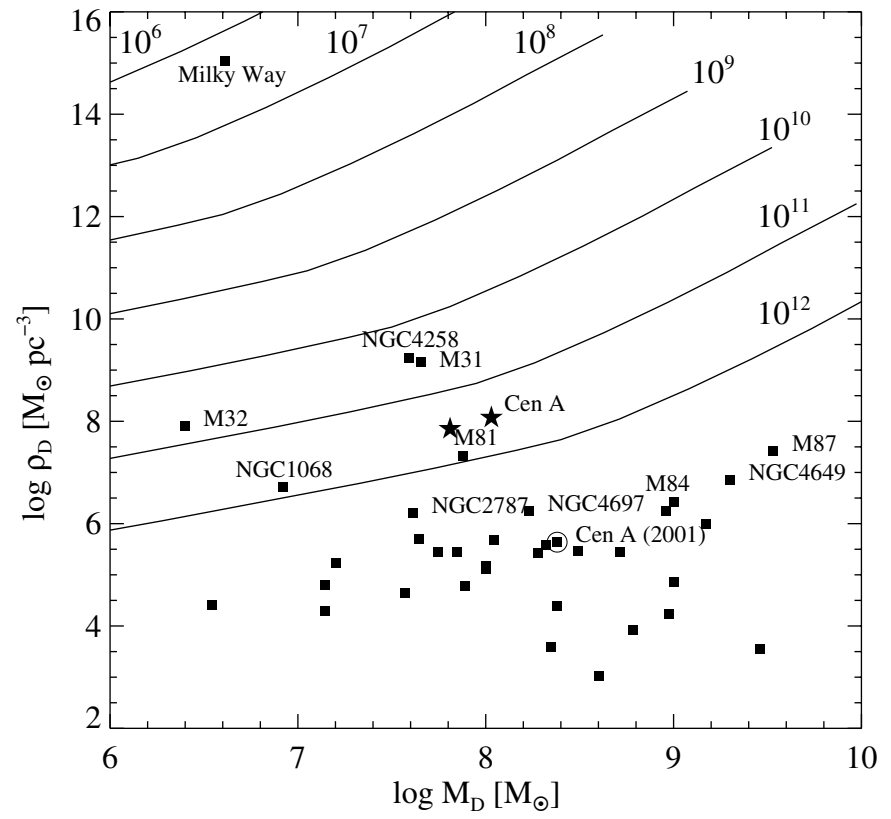

Fig. 29. Loci of dark cluster lifetimes (yr) in the mass-density plane (after Maoz 1998).

The two sets of data have spatial resolutions differing by almost a factor of five but provide independent and consistent measures of the BH mass, which are in agreement with our previous estimate based on the ISAAC data alone. The gas kinematical analysis provides a mass of $M_{\mathrm{BH}}=(1.1 \pm 0.1) \times 10^{8} M_{\odot}$ for an assumed disk inclination of $i=25 \mathrm{deg}$ or $M_{\mathrm{BH}}=$ $(6.5 \pm 0.7) \times 10^{7} M_{\odot}$ for $i=35 \mathrm{deg}$. Our new analysis improves the accuracy of the $\mathrm{BH}$ mass estimate with respect to Marconi et al. (2001) but does not substantially change their results.

We performed a detailed analysis of the effects on $M_{\mathrm{BH}}$ of the intrinsic surface brightness distribution of the emission line, a crucial ingredient in the gas kinematical analysis. We estimate that the associated systematic errors are no larger than 0.08 in $\log M_{\mathrm{BH}}$, comparable with statistical errors and indicating that the method is robust. However, the adopted intrinsic surface brightness distribution has a large impact on the value of the gas velocity dispersion. A mismatch between the observed and model velocity is not necessarily an indication of non-circular motions or kinematically hot gas, but is as easily due to an inaccurate computation arising from too course a model grid, or the adoption of an intrinsic brightness distribution which is too smooth.

The velocity dispersion in the ISAAC spectra is matched with a circularly rotating disk and also the observed line profiles and the higher order moments in the Hermite expansion of the line profiles, $h_{3}$ and $h_{4}$, are consistent with emission from such a disk. The velocity dispersion in the STIS data is slightly larger than expected from rotation however this mismatch can be reconciled when taking into account the signalto-noise ratio of the data and the possibility of using more peaked intrinsic surface brightness distributions described in the text. Nonetheless, STIS data provide an estimate of the $\mathrm{BH}$ mass consistent with ISAAC indicating that even an intrinsic velocity dispersion of the gas does not invalidate gas kinematical BH estimates.

To our knowledge, Centaurus A is the first external galaxy for which reliable $\mathrm{BH}$ mass measurements from gas and stellar dynamics are available. The agreement between our $M_{\mathrm{BH}}$ gas kinematical estimate and the similar estimate from stellar dynamics thus strengthens the reliability of both methods.

The BH mass in Centaurus A is in excellent agreement with the correlation with infrared luminosity and mass of the host spheroid but is a factor $\sim 2-4$ above the one with the stellar velocity dispersion. But this disagreement is not large if one takes into account the intrinsic scatter of the $M_{\mathrm{BH}}-\sigma_{\mathrm{e}}$ correlation.

Finally, the high HST spatial resolution allows us to constrain the size of any cluster of dark objects alternative to a $\mathrm{BH}$ to $r_{\bullet}<0.035(\simeq 0.6 \mathrm{pc})$. Thus Centaurus A ranks among the best cases for supermassive BHs in galactic nuclei.

Acknowledgements. We thank the anonymous referee for a thorough reading of the paper and insightful comments. We thank the editor, Steven Shore, for his useful suggestion about the BH in the Galactic Center.

\section{Appendix A: Computation of model kinematical quantities: the subsampling problem}

Marconi et al. (2003a) have shown how to derive the expressions used to compute the model quantities which must be compared with the observed ones. We summarize here their results and present a new way to compute model kinematical quantities which overcomes the "subsampling" problem described below.

Consider a reference frame $x y$ on the plane of the sky with the slit aligned along the $y$ axis. The slit center has coordinate $x=x_{0}$ and the points within the slit are characterized by $x_{0}-$ $\Delta x \leq x \leq x_{0}+\Delta x$ where $2 \Delta x$ is the slit width projected onto the plane of the sky. The spectrograph provides matrixes $S_{i j}$ where $i$ represents the wavelength direction and $j$ represents the direction along the slit. For $i=1, \ldots, N$ (where $N$ is the number of pixels along dispersion), the row $S_{i j}=\Psi_{j}\left(w_{i}\right)$ is the spectrum extracted at a given position $y_{j}$ along the slit and $w_{i}$ is the velocity at pixel $i$. Such spectrum is extracted from an aperture which, on the plane of the sky, has coordinates $x_{0}-$ $\Delta x \leq x \leq x_{0}-\Delta x$ and $y_{j}-\Delta y \leq y \leq y_{j}-\Delta y$, where $2 \Delta y$ is the pixel size along the slit.

The kinematical quantities can be computed as:

$\left\langle v_{j}\right\rangle=\frac{\left\langle v I_{j}\right\rangle}{\left\langle I_{j}\right\rangle}$

$\left\langle v_{j}^{2}\right\rangle=\frac{\left\langle v^{2} I_{j}\right\rangle}{\left\langle I_{j}\right\rangle}$

Marconi et al. (2003a) show that:

$\left\langle I_{j}\right\rangle=\int_{-\infty}^{+\infty} \Psi_{j}(w) \mathrm{d} w=2 \Delta w \int_{x_{1}}^{x_{2}} \mathrm{~d} x \int_{y_{1}}^{y_{2}} \mathrm{~d} y \iint_{-\infty}^{+\infty} \mathrm{d} x^{\prime} \mathrm{d} y^{\prime} \mathcal{P}$ 


$$
\begin{aligned}
\left\langle v I_{j}\right\rangle & =\int_{-\infty}^{+\infty} w \Psi_{j}(w) \mathrm{d} w \\
& =2 \Delta w \int_{x_{1}}^{x_{2}} \mathrm{~d} x \int_{y_{1}}^{y_{2}} \mathrm{~d} y \iint_{-\infty}^{+\infty} \mathrm{d} x^{\prime} \mathrm{d} y^{\prime} w_{0} \mathcal{P} \\
\left\langle v^{2} I_{j}\right\rangle & =\int_{-\infty}^{+\infty} w^{2} \Psi_{j}(w) \mathrm{d} w \\
& =2 \Delta w \int_{x_{1}}^{x_{2}} \mathrm{~d} x \int_{y_{1}}^{y_{2}} \mathrm{~d} y \iint_{-\infty}^{+\infty} \mathrm{d} x^{\prime} \mathrm{d} y^{\prime}\left[w_{0}^{2}+\frac{\Delta w}{3}+\sigma^{2}\right] \mathcal{P}
\end{aligned}
$$

$$
\begin{aligned}
& \overline{\overline{v I}}\left(x_{0}, y_{j}\right)=\int_{x_{0}-\Delta x}^{x_{0}+\Delta x} \mathrm{~d} x \int_{y_{j}-\Delta y}^{y_{j}+\Delta y} \mathrm{~d} y \overline{v I}(x, y) \\
& \overline{\overline{v^{2} I}}\left(x_{0}, y_{j}\right)=\int_{x_{0}-\Delta x}^{x_{0}+\Delta x} \mathrm{~d} x \int_{y_{j}-\Delta y}^{y_{j}+\Delta y} \mathrm{~d} y \overline{v^{2} I}(x, y)
\end{aligned}
$$

the final expressions can be written as:

$$
\begin{aligned}
\left\langle I_{j}\right\rangle= & \int_{-\infty}^{+\infty} \Psi_{j}(w) \mathrm{d} w=2 \Delta w \iint_{-\infty}^{+\infty} \mathrm{d} x^{\prime} \mathrm{d} y^{\prime} \mathcal{P} \overline{\bar{I}}\left(x_{0}, y_{j}\right) \\
\left\langle v I_{j}\right\rangle= & \int_{-\infty}^{+\infty} w \Psi_{j}(w) \mathrm{d} w \\
= & 2 \Delta w \iint_{-\infty}^{+\infty} \mathrm{d} x^{\prime} \mathrm{d} y^{\prime} \mathcal{P}\left[\overline{\overline{v I}}\left(x_{0}, y_{j}\right)-\tilde{v}(x) * \overline{\bar{I}}\left(x_{0}, y_{j}\right)\right] \\
\left\langle v^{2} I_{j}\right\rangle= & \int_{-\infty}^{+\infty} w^{2} \Psi_{j}(w) \mathrm{d} w=2 \Delta w \iint_{-\infty}^{+\infty} \mathrm{d} x^{\prime} \mathrm{d} y^{\prime} \mathcal{P}\left[\overline{\overline{v^{2} I}}\left(x_{0}, y_{j}\right)\right. \\
& \left.-2 * \tilde{v}(x) * \overline{\overline{v I}}\left(x_{0}, y_{j}\right)+\tilde{v}(x)^{2} * \overline{\bar{I}}\left(x_{0}, y_{j}\right)\right]
\end{aligned}
$$

way is to build regular grid in the $x, y^{\prime}$ plane and do the convolution with the PSF by using, for instance, the Fast Fourier Transform algorith (e.g. Press et al. 1992). After convolution, $\left\langle I_{j}\right\rangle,\left\langle v I_{j}\right\rangle$ and $\left\langle v^{2} I_{j}\right\rangle$ can then be computed by integrating the grids over the apertures from which observed spectra have been extracted. The requirement of regular grids is imposed by the FFT algorithm and the grid should be fine enough to accurately sample the PSF; for instance, with a Gaussian PSF, grids should have sampling steps less than or equal to $1 / 3$ of the Gaussian $\sigma$. The grid must also be large enough that edge effects are negligible over the aperture areas where the final integration is performed; for instance, with a Gaussian PSF, it is enough to extend the grid by $5 \sigma$ on all sides.

There is a problem of subsampling when the intrinsic surface brightness distribution $I$ is characterized by spatial scales which are much smaller that those of the PSF. A fine sampling of $I$ results then in grids which are very large (more than, eg, $1024 \times 1024$ points) and which make computational times too long. However, while it is very important to finely sample $I$ where there are the largest velocity gradients close to the $\mathrm{BH}$ location, this is a waste of computational resources where the velocity is slowly varying. A possibility to solve this problem is to invert the order of integration in the above expressions, i.e. first compute the integration over the apertures and then compute the FFT. In this way the small scales in $I$ are removed by the integration process and the sampling must be fine only with respect to the PSF. The formulas to be used after the inversion of the integrals are trivially computed. Defining

$$
\begin{aligned}
\bar{I}(x, y) & =I(x, y) \\
\tilde{v}(x) & =k *\left(x-x_{0}\right) \\
\bar{v}(x, y) & =v(x, y) I(x, y)+\bar{I}(x, y) \tilde{v}(x) \\
\overline{v^{2} I}(x, y) & =v^{2}(x, y) I(x, y)+\bar{I}(x, y) \tilde{v}(x)^{2}+2 * \overline{v I}(x, y) \tilde{v}(x)
\end{aligned}
$$

and

$$
\overline{\bar{I}}\left(x_{0}, y_{j}\right)=\int_{x_{0}-\Delta x}^{x_{0}+\Delta x} \mathrm{~d} x \int_{y_{j}-\Delta y}^{y_{j}+\Delta y} \mathrm{~d} y \bar{I}(x, y)
$$

where $\mathcal{P}=P\left(x_{0}-x^{\prime}, y_{j}-y^{\prime}\right)$. The integrals over the apertures can be computed, e.g., with adaptive grids in order to reduce computational times.

We now show the effects of subsampling on model values and how the above way of computation can solve them. We consider an intrinsic surface brightness distribution which is circularly symmetric on the plane of the sky and is described by a sum of two exponentials as

$I(r)=I_{0} \mathrm{e}^{-r / r_{0}}+I_{1} \mathrm{e}^{-r / r_{1}}$

where $r$ is the distance from the center and we assume the following parameter values: $I_{1} / I_{0}=0.01, r_{0}=0.05^{\prime \prime}$, and $r_{1}=1.00^{\prime \prime}$. The "observed" system has the same parameters as Centaurus A except for $M_{\mathrm{BH}}=10^{8} M_{\odot}, M / L=1$, and the gas disk is inclined by $45 \mathrm{deg}$ with respect to the line of sight. The rotating gas disk, which has an intrinsic constant velocity dispersion of $70 \mathrm{~km} \mathrm{~s}^{-1}$, is observed with ISAAC with $0.5^{\prime \prime}$ seeing $(F W H M)$. The slit is placed along the major axis of the disk.

The effects of subsampling are shown in Fig. A.1a. The solid line represent the model values computed using the method described above, while the other lines represents computations with the commonly adopted method and with different sampling steps. It is clear that the observed surface brightness distribution and velocity are very little affected by the subsampling problem. However the velocity dispersion can be underestimated even by a factor 1.5 for the largest sampling used which is nonetheless a factor 5 smaller than the PSF $\sigma$. The effects on velocity and velocity dispersion can also be seen if the $\mathrm{BH}$ location and peak of the surface brightness distribution are not located on one of the grid points. In Fig. A.1b the $\mathrm{BH}$ is offset by only $0.02^{\prime \prime}, 0.02^{\prime \prime}$ along and across the slit with respect to the center of surface brightness distribution. In this 

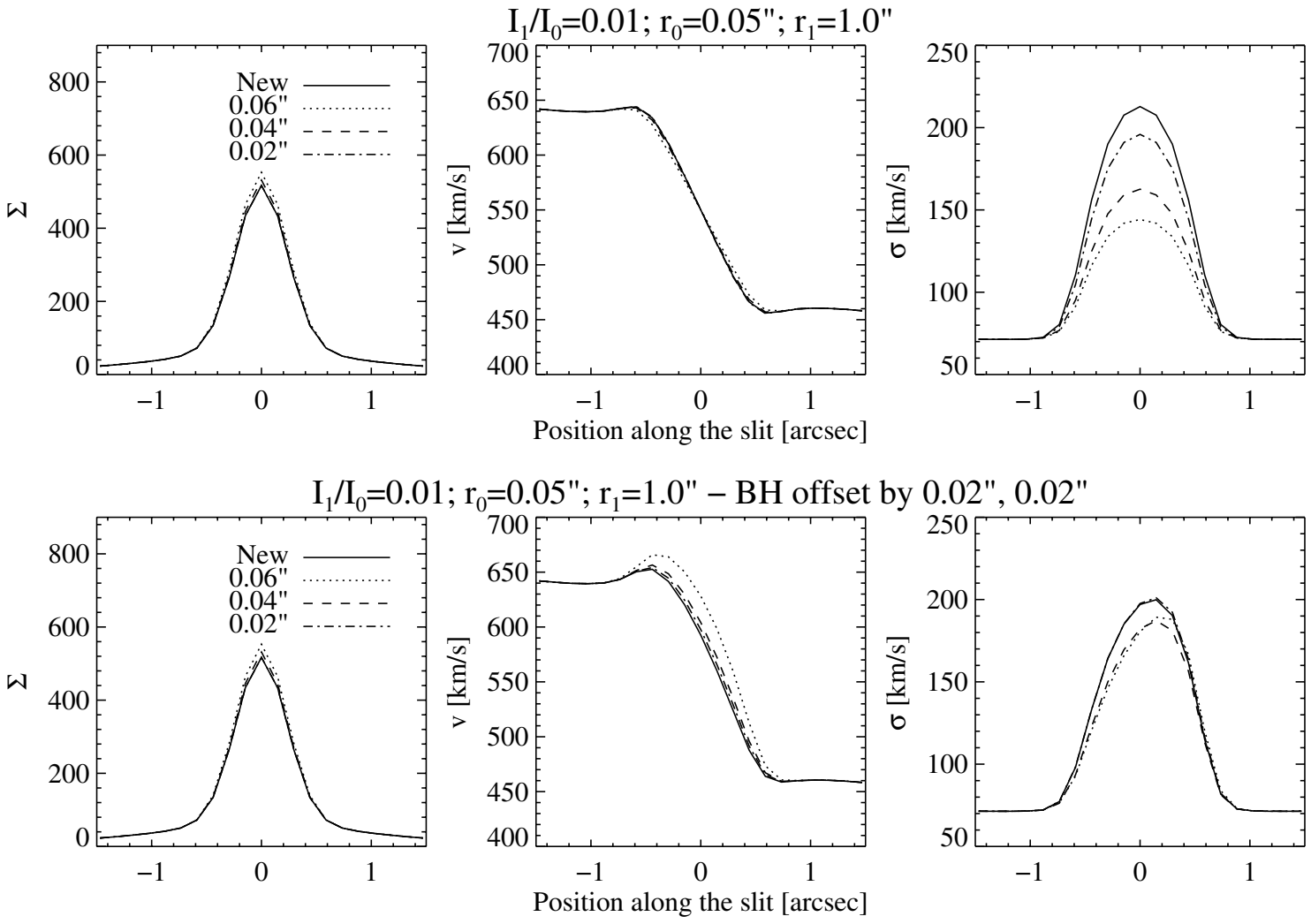

Fig. A.1. Top: instrument convolved kinematical quantities computed with different grid sampling. "New" indicates the computations with the new method described in the text. Bottom: as in the top panel but with the BH position offset by $0.02^{\prime \prime}, 0.02^{\prime \prime}$ with respect to the peak of the surface brightness distribution.

case all gas velocity dispersions are close to the correct value indicated by the solid line but the velocity values are starting to be affected by the sampling problem.

\section{References}

Avni, Y. 1976, ApJ, 210, 642

Axon, D. J., Marconi, A., Capetti, A., et al. 1998, ApJ, 496, L75

Barth, A. J., Sarzi, M., Rix, H., et al. 2001, ApJ, 555, 685

Binney, J., \& Tremaine, S. 1987, Galactic dynamics (Princeton, NJ: Princeton University Press), 747

Capetti, A., Axon, D. J., Macchetto, F. D., Marconi, A., \& Winge, C. 1999, ApJ, 516, 187

Capetti, A., Schreier, E. J., Axon, D., et al. 2000, ApJ, 544, 269

Cappellari, M., Verolme, E. K., van der Marel, R. P., et al. 2002, ApJ, 578, 787

Cappellari, M., \& Emsellem, E. 2004, PASP, 116, 138

Cardelli, J. A., Clayton, G. C., \& Mathis, J. S. 1989, ApJ, 345, 245

Chiaberge, M., Capetti, A., \& Celotti, A. 2001, MNRAS, 324, L33

Cretton, N., \& Emsellem, E. 2004, MNRAS, 347, L31

Di Matteo, T., Springel, V., \& Hernquist, L. 2005, Nature, 433, 604

Evans, D. A., Kraft, R. P., Worrall, D. M., et al. 2004, ApJ, 612, 786

Ferrarese, L., \& Merritt, D. 2000, ApJ, 539, L9

Ferrarese, L., \& Ford, H. 2005, Space Sci. Rev., 116, 523

Gebhardt, K., Bender, R., Bower, G., et al. 2000, ApJ, 539, L13

Genzel, R., \& Townes, C. H. 1987, ARA\&A, 25, 377

Goudfrooij, P., \& Christensen, J. A. 1998, STIS Instrument Science Report 98-29 (Baltimore: STScI)

Granato, G. L., De Zotti, G., Silva, L., Bressan, A., \& Danese, L. 2004, ApJ, 600, 580
Grandi, P., Fiocchi, M., Perola, C. G., et al. 2003, ApJ, 593, 160

Häring, N., \& Rix, H. 2004, ApJ, 604, L89

Hardcastle, M. J., Worrall, D. M., Kraft, R. P., et al. 2003, ApJ, 593, 169

Hui, X., Ford, H. C., Ciardullo, R., \& Jacoby, G. H. 1993, ApJ, 414, 463

Israel, F. P. 1998, A\&AR, 8, 237

Jarrett, T. H., Chester, T., Cutri, R., Schneider, S. E., \& Huchra, J. P. 2003, AJ, 125, 525

Karovska, M., Marengo, M., Elvis, M., et al. 2003, ApJ, 598, L91

Kim Quijano, J., et al. 2003, STIS Instrument Handbook, Version 7.0 (Baltimore: STScI)

Kormendy, J., \& Richstone, D. 1995, ARA\&A, 33, 581

Krist, J., \& Hook, R. 1999, The Tiny Tim User's Guide (Baltimore: STScI)

Leeuw, L. L., Hawarden, T. G., Matthews, H. E., Robson, E. I., \& Eckart, A. 2002, ApJ, 565, 131

Macchetto, F., Marconi, A., Axon, D. J., et al. 1997, ApJ, 489, 579

Maciejewski, W., \& Binney, J. 2001, MNRAS, 323, 831

Maoz, E. 1998, ApJ, 494, L181

Marconi, A., Schreier, E. J., Koekemoer, A., et al. 2000, ApJ, 528, 276

Marconi, A., Capetti, A., Axon, D. J., et al. 2001, ApJ, 549, 915

Marconi, A., \& Hunt, L. K. 2003, ApJ, 589, L21

Marconi, A., Axon, D. J., Capetti, A., et al. 2003a, ApJ, 586, 868

Marconi, A., Maiolino, R., \& Petrov, R. G. 2003b, Ap\&SS, 286, 245

Marconi, A., Risaliti, G., Gilli, R., et al. 2004, MNRAS, 351, 169

Menci, N., Cavaliere, A., Fontana, A., et al. 2003, ApJ, 587, L63

Merritt, D. 1997, AJ, 114, 228

Mirabel, I. F., Laurent, O., Sanders, D. B., et al. 1999, A\&A, 341, 667 
Press, W. H., Teukolsky, S. A., Vetterling, W. T., \& Flannery, B. P. 1992, Numerical recipes in FORTRAN. The art of scientific computing (Cambridge: University Press) 2nd edn.

Quillen, A. C., de Zeeuw, P. T., Phinney, E. S., \& Phillips, T. G. 1992, ApJ, 391, 121

Quillen, A. C., Graham, J. R., \& Frogel, J. A. 1993, ApJ, 412, 550

Quillen, A. C., McDonald, C., Alonso-Herrero, A., et al. 2001, ApJ, 547, 129

Rejkuba, M. 2004, A\&A, 413, 903

Schödel, R., Ott, T., Genzel, R., et al. 2003, ApJ, 596, 1015

Schreier, E. J., Capetti, A., Macchetto, F., Sparks, W. B., \& Ford, H. J. 1996, ApJ, 459, 535

Schreier, E. J., Marconi, A., Axon, D. J., et al. 1998, ApJ, 499, L143

Silge, J. D., Gebhardt, K., Bergmann, M., \& Richstone, D. 2005, AJ, 130,406
Soria, R., Mould, J. R., Watson, A. M., et al. 1996, ApJ, 465, 79

Tadhunter, C., Marconi, A., Axon, D., et al. 2003, MNRAS, 342, 861

Tingay, S. J., Preston, R. A., \& Jauncey, D. L. 2001, AJ, 122, 1697

Tremaine, S., Gebhardt, K., Bender, R., et al. 2002, ApJ, 574, 740

Valluri, M., Merritt, D., \& Emsellem, E. 2004, ApJ, 602, 66

van der Marel, R. P., \& Franx, M. 1993, ApJ, 407, 525

van der Marel, R. P., de Zeeuw, P., Rix, H.-W., \& Quinlan, G. D. 1997, Nature, 385, 610

van der Marel, R. P., \& van den Bosch, F. C. 1998, AJ, 116, 2220

Verdoes Kleijn, G. A., van der Marel, R. P., Carollo, C. M., \& de Zeeuw, P. T. 2000, AJ, 120, 1221

Verdoes Kleijn, G. A., van der Marel, R. P., de Zeeuw, P. T., Noel-Storr, J., \& Baum, S. A. 2002, AJ, 124, 2524

Yu, Q., \& Tremaine, S. 2002, MNRAS, 335, 965 